\title{
Structural and functional aspects of sensory-motor Interaction in the urinary bladder
}

Citation for published version (APA):

Biallosterski, B. T. (2015). Structural and functional aspects of sensory-motor Interaction in the urinary bladder. [Doctoral Thesis, Maastricht University]. Maastricht University.

https://doi.org/10.26481/dis.20150925bb

Document status and date:

Published: 01/01/2015

DOI:

10.26481/dis.20150925bb

Document Version:

Publisher's PDF, also known as Version of record

\section{Please check the document version of this publication:}

- A submitted manuscript is the version of the article upon submission and before peer-review. There can be important differences between the submitted version and the official published version of record.

People interested in the research are advised to contact the author for the final version of the publication, or visit the DOI to the publisher's website.

- The final author version and the galley proof are versions of the publication after peer review.

- The final published version features the final layout of the paper including the volume, issue and page numbers.

Link to publication

\footnotetext{
General rights rights.

- You may freely distribute the URL identifying the publication in the public portal. please follow below link for the End User Agreement:

www.umlib.nl/taverne-license

Take down policy

If you believe that this document breaches copyright please contact us at:

repository@maastrichtuniversity.nl

providing details and we will investigate your claim.
}

Copyright and moral rights for the publications made accessible in the public portal are retained by the authors and/or other copyright owners and it is a condition of accessing publications that users recognise and abide by the legal requirements associated with these

- Users may download and print one copy of any publication from the public portal for the purpose of private study or research.

- You may not further distribute the material or use it for any profit-making activity or commercial gain

If the publication is distributed under the terms of Article $25 \mathrm{fa}$ of the Dutch Copyright Act, indicated by the "Taverne" license above, 
Structural and Functional Aspects of Sensory-motor Interaction in the Urinary Bladder

Bart Tjomme Biallosterski 
Copyright $\odot 2015$ Bart Tjomme Biallosterski, Maastricht, 2015

ISBN: 978-90-9029194-9

Structural and functional aspects of sensory-motor interaction in the urinary bladder

Design, Printing \& Binding: 5nul8 Grafische Producties, Valkenburg ald Geul, The Netherlands - info@5nul8.nl

All rights reserved. No part of this thesis may be reproduced, stored in a retrieval center of any nature, or transmitted, in any form or by any means, electronic, mechanical, photocopying, recording or otherwise, without the permission of the author.

Financial support for publication of this thesis was kindly provided by: Stichting Wetenschappelijke Activiteiten Maastrichtse Urologie (WAMU); Astellas Pharma; Zambon Nederland; GlaxoSmithKline; Ipsen Farmaceutica; Goodlife Pharma; AbbVie; Eurocept Pharmaceuticals; Mundi Pharma. 


\title{
Structural and Functional Aspects of Sensory-motor Interaction in the Urinary Bladder
}

\author{
PROEFSCHRIFT \\ ter verkrijging van de graad van doctor aan de Universiteit Maastricht, \\ op gezag van de Rector Magnificus, Prof. Dr. L.L.G. Soete, \\ volgens het besluit van het College van Decanen, \\ in het openbaar te verdedigen \\ op vrijdag 25 september 20I5, om I4:00 uur \\ door
}

Bart Tjomme Biallosterski 
Promotoren:

prof. dr. Ph.E.V. Van Kerrebroeck

prof. dr. S.G.G. de Wachter (Universiteit van Antwerpen)

Copromotoren:

dr. G.A. van Koeveringe

dr. M.S. Rahnama'i

Beoordelingscommissie:

prof. dr. H.W.M. Steinbusch (Voorzitter)

Prof. dr. M.H.V. De Baets

dr. J. Heesakkers (Radboud Universiteit, Nijmegen)

prof. dr. T. Kessler (Balgrist University Hospital, Zürich)

dr. C. Meriaux

prof. dr. Y. Temel

The work presented in this thesis was performed at the department of Urology of the Maastricht University Medical Centre and School for Mental Health and Neuroscience (MHeNS) 


\section{Contents}

Chapter I: Introduction and Thesis Outline

Chapter 2: The Distribution of the Prostaglandin E Receptor Type 2 (EP2) in the Detrusor of the Guinea Pig.

Rahnama'i MS, Biallosterski BT, de Wachter SG, van Kerrebroeck PE

Chapter 3: Distribution and Sub-types of Afferent Fibre in the Mouse Urinary Bladder

Biallosterski BT, Rahnama'i MS, van Kerrebroeck PE, van Koeveringe GA,

Gillespie Jl, de Wachter SG.

Chapter 4: Non voiding Activity of the Guinea Pig Bladder

Biallosterski BT, van Koeveringe GA, van Kerrebroeck PE, Gillespie JI, de Wachter SG.

Chapter 5: Changes in Bladder Innervation in a Mouse Model of Alzheimer's Disease Biallosterski BT, de Wachter SG, van Koeveringe GA, van Kerrebroeck PE, de Vente J, Mulder MT, Gillespie JI.

Chapter 6: Changes in Voiding Behavior in a Mouse Model of Alzheimer's Disease Biallosterski BT, Prickaerts J, Rahnama'i MS, de Wachter SG, van Koeveringe GA, Meriaux C.

Chapter 7: General Discussion

Vallorisation

Summary

Nederlandse Samenvatting

Acknowledgements / Dankwoord

Curriculum Vitae 

Chapter I

General Introduction and Outline of the Thesis 

Structural and functional studies of the urinary bladder are necessary to elucidate the exact working mechanisms of storage and voiding of urine and the possible pathological disturbances in bladder control. The studies presented in this thesis concentrate on the sensory-motor interaction in the lower urinary tract possible roll in abnormal bladder behavior.

\section{The lower urinary tract}

The lower urinary tract comprises the bladder and the urethra and is supported by muscles and ligaments. The urethra contains both smooth and striated muscles. The bladder can be divided into two main components: the bladder body, which is located above the ureteral orifices, and the base, consisting of the trigone and urethrovesical junction. The bladder is essentially a hollow organ composed of separate layers, that include the detrusor muscle, a complex network of smooth muscle fibers and connective tissue that is responsible for bladder contractility. The inner lining of the bladder is called the urothelium, which also has a barrier function. The bladder muscular wall is formed of smooth muscle cells, also known as the detrusor muscle. The detrusor is structurally and functionally different from trigonal and urethral smooth muscle. The interaction between the smooth muscle cells in the bladder are important, as this will determine the behavior of the bladder wall and the effect bladder shape and intravesical pressure'. In many species, detrusor cells are oriented longitudinally in the outer and inner layers and circularly in the middle layer '. In the human detrusor, bundles of muscle cells are not clearly arranged in distinct layers, but run in various orientations, forming a meshwork ${ }^{1,2}$. The bundles vary in size, often a few millimeters in diameter, and are composed of several smaller sub-bundles.

\section{Bladder physiology}

The bladder serves as a reservoir for urine, but with intermittent expulsion capability of urine if fullness is perceived timely by the individual. This alternation between storage and voiding phases is known as the "micturition cycle". The storage and periodic elimination of urine depend on the coordinated activity of two functional units in the lower urinary tract: a reservoir (the urinary bladder) and an outlet consisting of the bladder neck, the urethra, and the urethral sphincter together with the pelvic floor muscles ${ }^{16}$. Coordination between these organs and structures is mediated by a complex neural control system located in the brain, spinal cord, and peripheral ganglia ${ }^{17}$. Hence, urine storage and voiding are highly dependent on central nervous system pathways. Due to the complexity of the neural mechanisms regulating the lower urinary tract, micturition regulation is influenced by a wide variety of changes due to for example injuries, diseases, and chemicals that affect the nervous system. As a consequence, neurologic mechanisms are an important consideration in the diagnosis and treatment of voiding disorders ${ }^{18}$. Effective storage implies that the reservoir is fully relaxed, and voiding indicates that the whole organ is contracting synchronously. The presumed general congruence of the reservoir (i.e. the whole organ is either relaxed or contracting) led to 
Structural and Functional Aspects of Sensory-motor Interaction in the Urinary Bladder

suggestions that the bladder musculature functions as a "functional syncytium", so that behavior of any part of the bladder could be taken as indicative of the state of the whole organ. However, this is incorrect, as clearly indicated by the presence of micromotions (localized contractions and elongations) during urine storage ${ }^{19}$. In order to understand the reservoir function of the bladder, and potentially voiding as well, information is needed on the contractile properties of the organ as a whole and how this may translate into pressure and sensation.

\section{Neuronal control}

The lower urinary tract alternates between "storage" and "voiding" phases, as a result of two synergic coordinated states of the bladder and the outlet (urethra and pelvic floor musculature).

Storage of urine is the result of the absence of global bladder contraction, enabling the organ to function as a low pressure reservoir. During the storage phase, there is probably background descending inhibition from higher central nervous system centers (Figure I) ${ }^{20}$. During storage, the bladder outlet (particularly the urinary sphincter) remains fully active, preventing flow of urine. Simultaneously, sympathetic nervous system storage reflexes, result in contraction of the bladder neck, and contribute to bladder relaxation at a peripheral level, thereby allowing the bladder to accommodate urine at low pressure throughout the filling phase ${ }^{21}$. Detrusor contractility is also inhibited by sympathetic reflexes, increasing tension in the smooth muscle around the bladder neck and urethra and inhibiting parasympathetic transmission through an action on either a spinal or ganglionic level ${ }^{22}$.

In contrast, bladder emptying (the voiding phase) is under parasympathetic (semi-voluntary) control. Neuronal control of micturition is complex, involving communication between many parts of the central nervous system (such as cerebral cortex, limbic system, hypothalamus, thalamus, basal ganglia, cerebellum, brain stem and spinal cord) and between the central and peripheral nervous system. The higher centres exhibit a continuous inhibitory effect on the pontine micturition centre. It is release of this inhibition that results in the 'permission to void'. At the level of spinal cord, reflex (autonomic) pathways are also active during bladder filling and facilitate storage of urine.

There are three crucial lower motor nuclei involved in lower urinary tract function; the parasympathetic nucleus of the sacral spinal cord controlling the detrusor, Onuf's nucleus in the sacral spinal cord controlling the urethral sphincter, and the sympathetic nucleus in the thoracolumbar spinal cord controlling the bladder neck. Maintenance of the synergic coordination of these nuclei, as appropriate for storage and voiding, is regulated by the upper motor neurons. For voiding, it is the pontine micturition center that "switches" the relevant spinal nuclei into the appropriate state. When voiding is initiated, bladder contraction results as a consequence of activation of the parasympathetic nucleus, while opening the urethra is achieved by inhibiting the sympathetic and Onuf's nucleus, respectively allowing the bladder neck and 
urethral sphincter to relax ${ }^{23}$. Afferent (sensory) information is sent via the pelvic nerve and the spinal cord to the periaqueductal grey, and onwards to higher centers responsible for conscious awareness (sensation). The periaqueductal grey relays on to the pontine micturition center, and receives the permissive input from the cerebrum which determines the timing of effective initiation of voiding ${ }^{23}$.

Age-related white matter lesions, also called vascular dementia, and Alzheimer's disease are both common causes of dementia and are known to be independent risk factors for neurogenic OAB and urinary incontinence. It remains uncertain which problem is a more significant contributor to neurogenic $O A B$ and incontinence in elderly adults with dementia ${ }^{24}$.

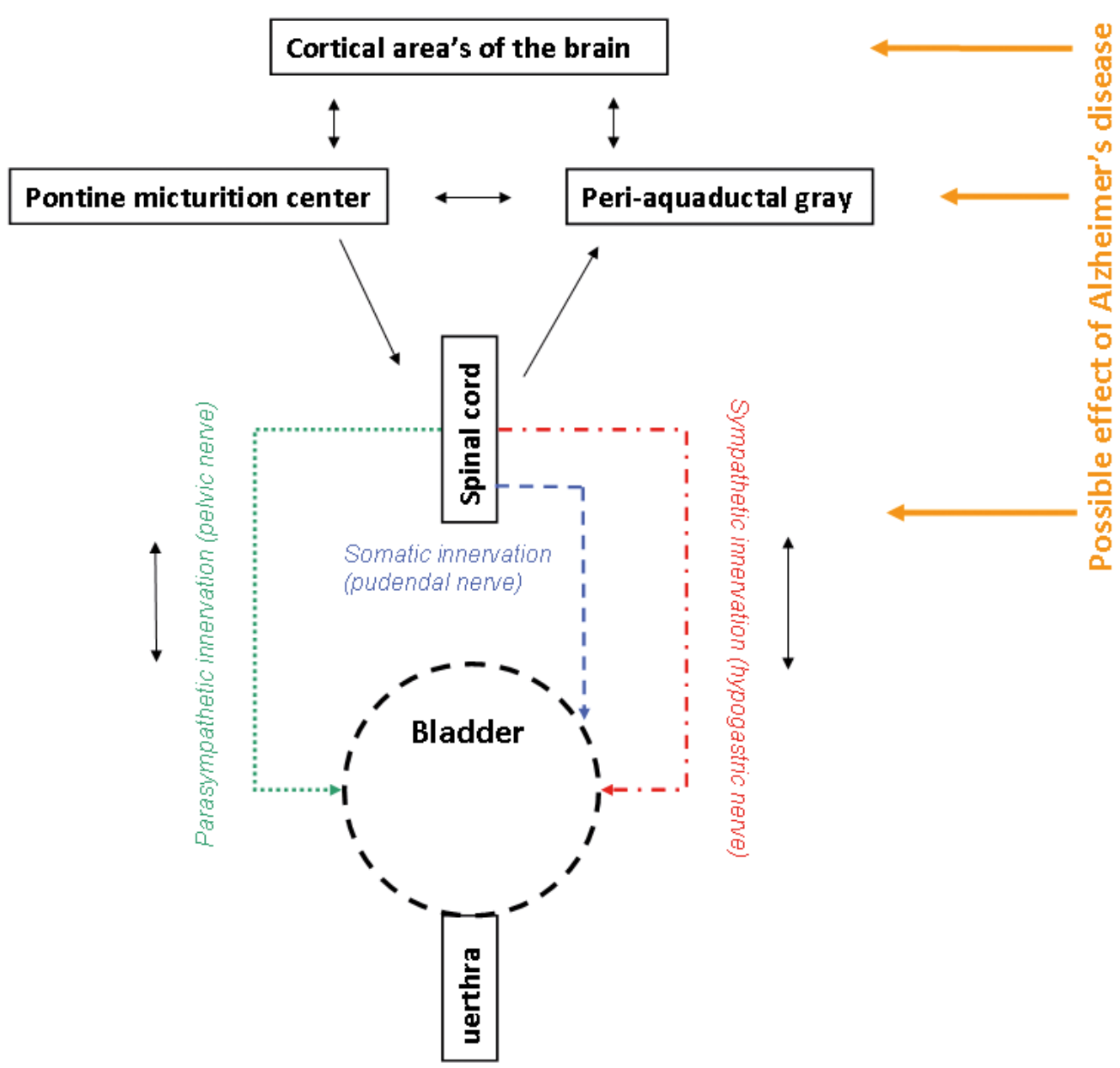

Figure I. Neuronal control of the bladder and possible effects of Alzheimer's disease 


\section{Non-voiding contractions}

For decades, the bladder was considered to be "silent", e.g. without any transient pressure increases during the filling phase. Presence of contractions during filling phase was marked as detrusor overactivity (DO) and considered to be pathological. The general assumption was that the bladder is inactive during the storage phase, with limited motility and change in the intravesical pressure. After all, motility and pressure fluctuations appear counter-intuitive, since they would seemingly counteract reservoir function. However, in 1892, Sherrington has reported small phasic fluctuations in pressure of the cat bladder prior to micturition ${ }^{25}$. Subsequently, in many species ${ }^{26,27}$, phasic fluctuations in pressure have been observed under conditions modelling the storage phase, generally increasing in amplitude and frequency as the bladder is filled ${ }^{25}$. In addition, bladder wall movements (micromotions) have been detected in various species, including the guinea-pig, rat, pig and human ${ }^{28-32}$. Most studies have used isolated whole bladders or bladder segments. Bladders of small animal species can be maintained in vitro using transmural oxygenation by diffusion. However, bladders of larger species, like humans have a larger tissue volume and therefore artificial perfusion is needed to maintain oxygenation and prevent ischaemia ${ }^{30}$. This is one of the reasons why studies with larger bladders are lacking. Involuntary detrusor contractions may lead to symptoms such as urgency, increased frequency of voiding and incontinence, which can affect quality of life and the ability to work and function normally. However, ambulatory urodynamic measurements have shown non voiding detrusor contractions during the filling phase in up to $69 \%$ of healthy volunteers ${ }^{33,34}$, suggesting that these contractions may be part of a normal physiological mechanism. Currently, the differences in non-voiding contractions between normal and pathological states are unknown. Non-voiding contractions have been shown to occur in vitro in the isolated whole animal bladder preparation ${ }^{27}$, and in that model, the non-voiding contractions can be increased by adenosine triphosphate (ATP), nitric oxide (NO), acetylcholine and increasing bladder volume or decreased by phosphodiesterases (PDE) and calcitonin gene related peptide (CGRP) ${ }^{29,35-39}$. These data suggest that non-voiding contractions are generated and processed, at least in part, within the bladder wall itself. In rats, the non-voiding contractions have been studied in vivo, showing that they increase in amplitude and frequency as the bladder fills ${ }^{40}$. The exact role or purpose of non-voiding contractions is still unknown, but if they are able to induce afferent activity, they could serve as a "primary sensory mechanism" within the lower urinary tract. Enhancement of this activity might then result in increased bladder sensations leading to urgency ${ }^{41}$.

\section{Definitions}

According to the International Continence Society (ICS), lower urinary tract symptoms (LUTS) can be divided into storage symptoms, voiding symptoms and postmicturition symptoms. LUTS in male patients is usually attributed to benign prostatic enlargement and outlet obstruction. LUTS in the female are predominantly attributed to the overactive bladder syndrome $(O A B)^{3-5}$. The 
definition of $O A B$ includes symptoms of urinary urgency, with or without urgency incontinence, usually accompanied by urinary frequency (generally accepted as $\geq 8$ micturitions in 24 hours) and nocturia ${ }^{3-5}$ OAB occurs in both sexes and is in about $50 \%$ of the patients accompanied by detrusor overactivity (DO) which is defined as uncontrolled contractions of the bladder (detrusor muscle) during bladder filling. About half of all individuals with DO, detected during conventional urodynamic studies, complain of clinical symptoms ${ }^{6,7}$ which means that, $O A B$ and DO have about a $50 \%$ overlap. In order to diagnose $O A B$, other specific causes (e.g. infection, stones, cancer) should be excluded.

Urgency is defined as a sudden and compelling desire to void, which in patients with $O A B$, cannot be postponed ${ }^{8}$. Urgency urinary incontinence occurs when a patient cannot reach the toilet in time. Urgency incontinence is considered the most bothersome symptom of $O A B$ and occurs in about $20 \%$ of men and $40 \%$ of women with the diagnosis OAB (indicated as OAB-wet) ${ }^{9}$. This leaves about $80 \%$ of male and $60 \%$ of female patients that present with symptoms of OAB without having urgency urinary incontinence (indicated as OAB-dry) ${ }^{10}$.

Detrusor underactivity is defined as a voiding contraction of reduced strength and/or duration, which prolongs urination and/or prevents complete emptying of the bladder within a 'normal' period of time ". Detrusor underactivity is associated with voiding and postmicturition urinary symptoms, and can predispose to urinary infections and acute urinary retention. The etiology of detrusor underactivity is influenced by multiple factors, including ageing, bladder outlet obstruction, neurological disease, and autonomic denervation. The true prevalence of this condition remains unknown ${ }^{12}$, as most data come from referral populations. Urodynamic testing is used to diagnose the condition, either by assessing the relationship between bladder pressures and urinary flow, or by interrupting voiding at request, in order to measure detrusor pressure change under isovolumetric conditions.

Urinary incontinence generally occurs if the pressure in the bladder unintentionally exceeds that within the urethra during the filling phase of the micturition cycle. It may occur as a result of uninhibited contractions of the detrusor muscle, e.g. detrusor overactivity. Other important reasons for urinary leakage include relaxation of the urethral sphincter (urethral instability) or incompetence of the urethral closure mechanism (e.g. stress urinary incontinence). The causes of urinary incontinence are listed in table $\mathrm{I}$.

Stress urinary incontinence (SUI) is common in women, particularly following childbirth. In men it more frequently occurs when they have had a prostatectomy or other pelvic surgery. SUI is characterized by involuntary urinary leakage, in the absence of activity of the detrusor muscle, when there is an increase of intra-abdominal pressure such as lifting, coughing, sneezing and running. It occurs as a result of a variable combination of intrinsic urethral sphincter muscle weakness and an anatomical defect in the urethral support, leading to insufficient closure pressure in the urethra during physical stress.

Urgency urinary incontinence (UUI) is leakage of urine due to unintended detrusor muscle 
contraction. Patients suffering from UUI feel a sudden and strong desire to void but are not able to reach the toilet in time. Often these contractions occur regardless of the amount of urine that is in the bladder. In general, no specific cause of UUI can be identified. Although UUI may occur in both sexes and at any age, it is more common in women and the elderly.

Mixed incontinence is the term used to describe individuals with stress and urgency urinary incontinence. This type of incontinence occurs primarily in women (as stress urinary incontinence is rare in men) and accounts for approximately one third of all cases of urinary incontinence in women ${ }^{13}$.

Table 1: Causes of urinary incontinence

- Overactive bladder

- Urethral sphincter incompetence

- Bladder outlet obstruction

- Congenital laesions (epispadias, ectopic ureter, spina bifida)

- Bladder outlet fistulas

- Temporary causes (urinary tract infection, faecal impaction, confusional states)

- Neurological impairment (e.g. cerebral infarction, spinal cord injury, Alzheimer's disease)

Detrusor overactivity is further divided into idiopathic detrusor overactivity (IDO) or DO with no evident cause, and neurogenic DO (NDO), which is DO in a patient with an underlying neurological condition (common with degenerative diseases such as multiple sclerosis or after traumatic events as spinal injury) ${ }^{14}$. For the diagnosis of IDO all known causes of DO (e.g. infection, stones, cancer, NDO) should have been excluded.

NDO is likely to represent an excess of sensory stimulation from the bladder, a pathologically lowered sensory central threshold for afferent stimulation from the bladder, or a dyssynchronous relationship between afferent signalling and central inhibition of bladder contractions. This can lead to increased voiding frequency, reduced interval between voids and reduced volumes voided. Functional magnetic resonance imaging in healthy female controls suggests that neurological responses increase steadily with bladder filling ${ }^{15}$. Normal bladder filling sensations are mapped mainly in the insula, shifting anteriorly as sensation becomes stronger and gradually unpleasant. In subjects with poor bladder control, sensations and brain responses are abnormal. Responses are relatively limited at low bladder volumes (with mild sensation), but become exaggerated (with strong sensation) above a certain volume threshold, even when there is no actual detrusor overactivity ${ }^{15}$. Consequently, it appears that increased bladder afferent activity cannot account solely for abnormal responses during filling but a more complex organization in which the nature of afferent signals or the handling or the signals in the brain appears to be abnormal ${ }^{15}$. Neurological deficit (e.g. Multiple sclerosis, Parkinson's disease, stroke and spinal trauma) can, therefore, also cause DO and is indicated as 'neurogenic detrusor overactivity'. 


\section{The local bladder control mechanism}

The bladder appears to have a peripheral control mechanism located in the bladder wall, formed by the co-operative interaction of numerous regulatory cell types (mainly interstitial cells and peripheral neurones) ${ }^{19}$. Moreover, the urothelium is an integral part of a 'chemical' sensory system and plays a role in generating and modulating bladder sensation. Urothelial cells are known to express a variety of receptors or ion channels that are responsive to external agents and mechanical or thermal changes. These include: receptors to bradykinin, 15 trkA, and p7516; purines (P2X and P2Y); ${ }^{42}$ noradrenaline; ${ }^{43}$ acetylcholine (nicotinic and muscarinic); ${ }^{44}$ protease activated receptors; ${ }^{45}$ epithelial $\mathrm{Na}^{+}$channels $(\mathrm{ENaC}),{ }^{46}$ the $\mathrm{Deg} / \mathrm{ENaC}$ family ${ }^{47}$ and a number of transient receptor potential (TRP) channels (TRPVI, TRPV2, TRPV4, TRPM8, TRPAI). ${ }^{48-50}$ Stimulation of these urothelial sensor molecules can lead to the release of substances, such as ATP, PGs, acetylcholine, and $\mathrm{NO},{ }^{51}$ which have excitatory and inhibitory actions on afferent nerves located close to or in the urothelium. ${ }^{50,52}$

A variety of cells produce prostaglandins (PG) via the cyclooxygenase pathway in the bladder.Both the lamina propria and the muscle layers can release PG in response to various physiological and pathological stimuli, including stretch of smooth muscle and sensitization by noxious stimuli ${ }^{53-55}$. The receptors for prostaglandin $\mathrm{E}_{2}\left(\mathrm{PGE}_{2}\right)$, the most common prostaglandin in the bladder, are classified into four types: EPI, EP2, EP3 and EP4 ${ }^{53}$.

Prostaglandin receptors EPI and EP2 are shown to be expressed in the lamina propria indicating that this part of the bladder wall can respond to prostaglandin $\mathrm{E}_{2}{ }^{56}$. Both EPI and EP2 receptors have been identified on guinea pig smooth muscle cells, as well as on vimentin positive interstitial cells ${ }^{57-59}$. Intra-arterial administration of $\mathrm{PGE}_{2}$ to the urinary bladder is known to enhance the micturition reflex ${ }^{60}$. Cyclooxygenase inhibiting drugs, can alter normal voiding function as well as bladder hyperactivity induced by chemical irritation of the lower urinary tract in rats ${ }^{61}$. PG might act at various sites to regulate voiding. In the muscle layer, it has been suggested that PG can be co-released with acetylcholine at efferent nerve endings and directly contribute to muscle excitation ${ }^{62}$. Another suggestion is that PG might have an indirect effect on pre-synaptic motor terminals to affect the release of excitatory transmitters ${ }^{63}$. Furthermore, PG is suggested to inhibit acetylcholine esterase ${ }^{64}$ or enhance myogenic bladder activity ${ }^{65}$.

\section{Pathophysiology of bladder dysfunction}

Accumulating evidence indicates that aberrant afferent activity plays an important role in $O A B^{23}$. During bladder filling, sensory information is relayed to the central nervous system by the pudendal, pelvic and hypogastric nerves (Figure I). Each of these nerves contains different types of afferent fibers, which can be identified and differentiated structurally and functionally. They consist of small myelinated $A \delta$ fibers, mainly located in detrusor muscle, and unmyelinated $C$ nerve 
Structural and Functional Aspects of Sensory-motor Interaction in the Urinary Bladder

fibers innervating all layers of the bladder wall. These types can functionally be distinguished based on the conduction velocity, or their response to mechanosensitive or chemosensitive stimuli. However, no functional relationship between conduction velocity and receptor type has been described ${ }^{66}$. Histological studies have shown that bladder afferent nerve fibers originate from the dorsal root ganglia and contain multiple neuropeptides, e.g. Substance P (SP), Neurofilament protein (NF), neuronal Nitric Oxide synthase (nNOS) and CGRP ${ }^{23}$. The exact distribution of terminal endings of these peptide-containing afferents is still largely unknown but they appear to be more densely present in the lamina propria just beneath the urothelium ${ }^{67}$. Nerve fibers in the lamina propria near the urothelium are considered to be solely afferent fibers as no efferent system has yet been identified ${ }^{67}$. Furthermore, the afferent innervation of the bladder neck and trigone is higher in density than the lateral wall and dome of the bladder ${ }^{68}$. Regional variation in the suburothelial cholinergic innervation has been observed in the guinea pig urinary bladder, suggesting functional differentiation between the lateral wall and bladder base ${ }^{69}$. Moreover, co-localization of different neuropeptides has been shown also suggesting a functional heterogeneity ${ }^{70}$. This may explain why some fibers respond to a wide range of mechanical and chemical stimuli, such as stretch, pain, chemical modulation and motor/sensory elements ${ }^{71}$. In the mouse bladder, using electrophysiological techniques, at least four functionally different types of afferent nerve fiber have been identified ${ }^{72}$. Based on their responses to mechanical stimuli (perpendicular von Frey probing, urethral stroking, stretch), urothelial, muscular/urothelial, muscular, and serosal afferents were found. Structurally, the presence of CGRP and nNOS nerve fibers in the lamina propria have been studied but no specific attention was paid to regional differences ${ }^{73}$. Myelinated sensory A-delta fibers, which are located primarily within the detrusor smooth muscle layer, respond primarily to detrusor stretching during the phase of bladder filling and convey sensations of fullness. Unmyelinated sensory C-fibers are more widespread, and reside in the muscle, close to the urothelium, in the lamina propria, and directly adjacent to the urothelial cells ${ }^{74}$. C-fibers have a higher mechanical threshold than $A$ delta fibers and can be activated by a variety of neurotransmitters and chemical mediators released by the detrusor and urothelium, including ATP, neurokinins, such as substance $P$ and neurokinin $A$ and nerve growth factor (NGF) ${ }^{74,75}$.

$\mathrm{C}$-fibers are generally quiescent during normal voiding, but they may be critical for symptom generation in pathologic states such as $O A B$. These fibers demonstrate remarkable plasticity. Following neurologic or possibly inflammatory insult, C-fibers become the important afferent route to the spinal tract, carrying impulses involved in the (pathological) micturition reflex ${ }^{74,75}$.

The prevalence of OAB increases with age ${ }^{10,76}$ (Figure 2), and is estimated to be close to $12 \%$ in the western community ${ }^{10}$, $11-14 \%$ in Japan ${ }^{77}$ and $14-23 \%$ in Brazil ${ }^{78}$. More recent studies have estimated the prevalence of OAB in Europe ${ }^{79}$ to be around $17 \%$ and in the USA around $26-33 \%$ in men and $27-46 \%$ in women ${ }^{80}$. Many patients affected by $O A B$, are thought, not to seek medical treatment because of embarrassment. 
$O A B$ imposes a considerable financial burden on the patients affected, their families and the society ${ }^{81},{ }^{82}$. Costs are related to pad use, pharmacotherapy, catheters, physician visits, outpatient and inpatient attendances as well as intangible costs, such as time and loss of productivity ${ }^{83}$. The economic burden is significant and is comparable to the cost of breast cancer, osteoporosis, and diabetes ${ }^{81}$. One study in Germany demonstrated the cost of OAB at 3.57 billion Euros per year, close to yearly cost of diabetes, being 5. I billion Euros and that of dementia being 5.6 billion Euros per year ${ }^{84}$.

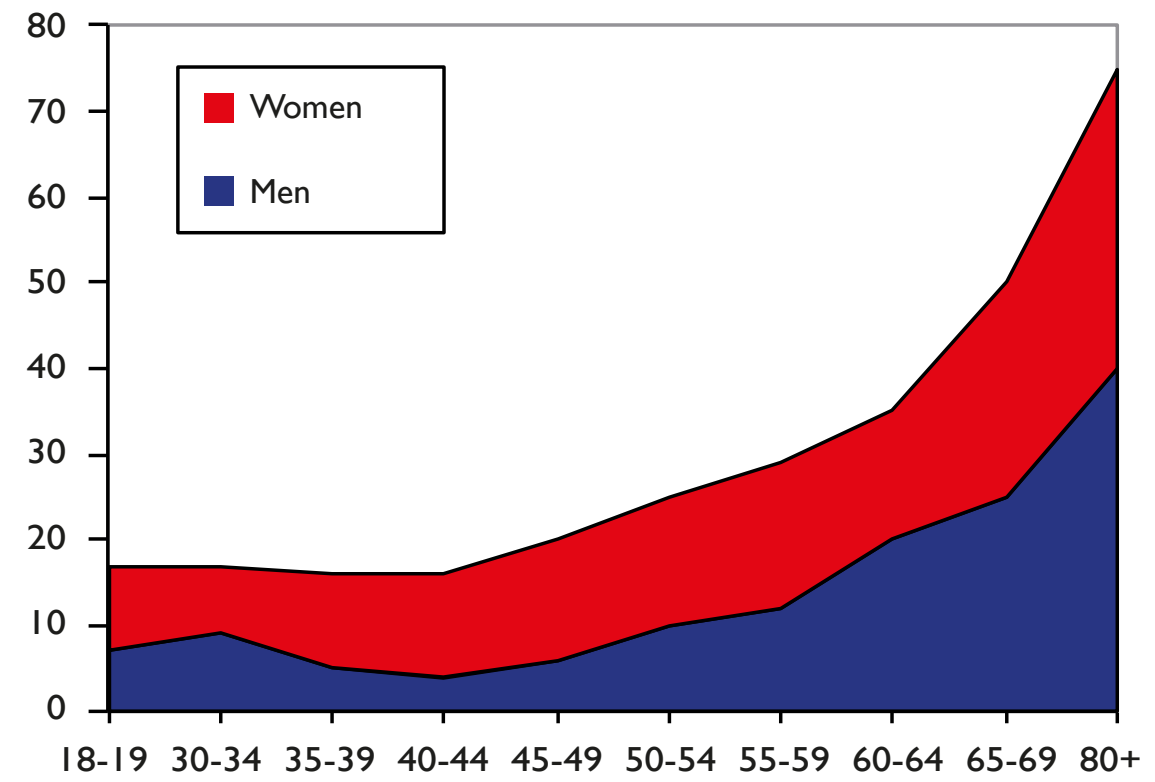

Figure 2. The prevalence of $O A B^{79}$

\section{Alzheimer's disease and bladder dysfunction}

Dementias, including Alzheimer's disease, are disabling conditions that can significantly affect the quality of life of those who suffer. The most common type of urinary incontinence in patients with Alzheimer's disease is urgency urinary incontinence ${ }^{85}$. For many such patients incontinence and more so urgency are major additional problems, which seriously affects their care and lifestyle ${ }^{86,87}$. Incontinence often emerges when dementia has developed into a moderate disease stage ${ }^{88}$.

It is generally accepted that dementia related incontinence is linked to cognitive impairment and reduced awareness. People with sufficient cortical function initiate voiding at an acceptable moment after going to a suitable location with the appropriate measures taken i.e. in a toilet and partially undressed. Patients with dementia and especially Alzheimer's disease, can demonstrate uninhibited and unembarrassed voiding at an inappropriate place and/or time. This behavior is often referred to as functional incontinence, and could be caused by lack of control at several levels. The general belief is that this type of incontinence is not the result of an abnormality in the lower urinary tract or its 
innervation, but from deficiencies in cholinergic neurotransmission in the cortical and subcortical areas of the CNS which then consequently leads to problems in locomotion and cognitive decline ${ }^{89}$. Cognitive severity and decline in dementia have been shown to significantly correlate to behavioral alterations, such as anxiety ${ }^{90}$ and several studies have linked anxiety to voiding dysfunction ${ }^{90,91}$. Possible changes in the lower urinary tract of patients with dementia that might contribute to and exacerbate the problem, has been less emphasized. Many patients with AD show signs of detrusor overactivity (DO) upon cystometric evaluation ${ }^{87,92}$. Dysfunctions in the integrated (neuro)physiology of the bladder itself may contribute to this disabling condition. This is supported by structural changes found in the bladder of a transgenic Alzheimer mouse model ${ }^{93}$.

\section{Animal Models}

Legal, ethical and moral issues do not allow work of necessary bladder experiments to be carried out in human subjects. Moreover, human bladder tissue is difficult to gain, especially from patients affected with Alzheimer's disease. Therefore, for the research presented in this thesis, the guinea pig bladder was used as study model. The guinea pig bladder has similarities is structure as well as cystometric parameters to the human bladder ${ }^{94-97}$ and has been used in many other experiments in our research laboratorium ${ }^{56,58,98,99}$. The use of the same animal model makes comparison of data easier. Therefore, guinea pig bladder was the first choice for further experiments.

Experimental investigations concerning dementia and bladder dysfunction are difficult on moral and ethical grounds. However, transgenic animals have provided an important approach to understand the changes in dementia. Mice have been bred, in which specific mutations have been engineered which mimic, in part, the changes seen in dementia including Alzheimer's disease. One such model involves a double mutation: one mutation is to the Amyloid Precursor Protein (APP) which includes both the Swedish and London mutations (KM6706/67INL and V7I7I) ${ }^{100}$ and a second mutation to the PSIMI46L ${ }^{101}$. This double transgenic mice (APP ${ }^{\mathrm{SL}} / \mathrm{PSI}^{\mathrm{MI}}{ }^{46 \mathrm{~L}}$ ) exhibit numerous changes in the central nervous system ${ }^{100-103}$, including increased amyloid deposits leading to plaque formation, with enlarged cholinergic terminals associated with and confined to the periphery of compact plaques. The model also shows activated astrocytes and microglia clusters. Since many of these changes are seen post mortem in patients with Alzheimer's disease, the model is considered appropriate to study the cellular and molecular aspects of Alzheimer's disease and its consequences ${ }^{104}$. The studies presented in this thesis used the aforementioned double transgenic mouse model in order to explore the peripheral changes in the bladder that might contribute to the overall patho-physiological changes.

The first study presented, focused on the distribution of nerve fibers in the bladder wall of the guinea pig, using nitric oxide synthase (nNOS), which is widely distributed in the lower urinary tract and may be important as an inhibitory neurotransmitter released by afferent nerves ${ }^{23,105}$. Several factors are thought to be responsible for the altered voiding behavior seen in patients with 
Alzheimer's disease. To gain insight into bladder function, rodents are often studied using metabolic cages, allowing voiding frequency and volume to be measured while urine can be collected and analyzed ${ }^{106,107}$. However, this method does not provide any information on the voiding behavior of freely moving animals. As Alzheimer's disease is correlated with both anxiety and incontinence ${ }^{87}$. The second study investigated both the affective and the voiding behavior in an APPSLxPSImutated mouse model of Alzheimer's disease.

\section{Research questions}

This thesis aims to gain a better understanding of structural and functional aspects of the sensory information and the motor output in the urinary bladder. Several experiments have been designed and carried out and the results are presented.

The first part of the thesis focuses on the morphological and structural changes in the urinary bladder wall of the guinea pig. The key research questions are the spatial relationship and distribution of the prostaglandin E receptor type 2 (EP2) in the guinea pig urinary bladder, as well as the different subtypes of afferent nerve fibers, in the mouse bladder wall.

The second part of the thesis concentrates on functional bladder studies in mice. The effect of filling rate and intravesical filling on non-voiding contractions, as well as the differences in the structure of bladders from transgenic mouse models of Alzheimer's disease with age matched control animals were studied. In addition, the urinary marking behaviour in relation to affective behaviour in an Alzheimer model mouse is evaluated.

\section{Thesis outline}

This thesis consists of seven chapters and has two main parts. After a general introduction as displayed in chapter I, the first part follows, presenting three studies. Chapter 2 deals with the prostaglandin $E_{2}$ receptor (EP2) distribution in the guinea pig detrusor muscle layers and in chapter 3, a study of the different types of afferent nerve fibres in the lamina propria of the mouse bladder is presented. Chapter 4 is a functional study of the non-voiding contractions of the guinea pig bladder.

The second part of the thesis focuses on bladder dysfunction in Alzheimer's disease. In chapter 5 a study is presented on changes in bladder innervation in a mouse model of Alzheimer's disease and chapter 6 deals with changes in voiding behaviour in the same model. A general discussion is presented in chapter 7 . 
Structural and Functional Aspects of Sensory-motor Interaction in the Urinary Bladder

\section{References}

I. Andersson, K.E. \& Arner, A. Urinary bladder contraction and relaxation: physiology and pathophysiology. Physiological reviews 84, 935-986 (2004).

2. Drake, M.J., Gardner, B.P. \& Brading, A.F. Innervation of the detrusor muscle bundle in neurogenic detrusor overactivity. BJU international 91, 702-710 (2003).

3. Abrams, P., et al. The standardisation of terminology in lower urinary tract function: report from the standardisation sub-committee of the International Continence Society. Urology 61, 37-49 (2003).

4. Abrams, P., et al. Reviewing the ICS 2002 terminology report: the ongoing debate. Neurourology and urodynamics 28, 287 (2009).

5. Haylen, B.T., et al. An International Urogynecological Association (IUGA)/International Continence Society (ICS) joint report on the terminology for female pelvic floor dysfunction. Neurourology and urodynamics 29, 4-20 (2010).

6. Abrams, P., et al. Fourth International Consultation on Incontinence Recommendations of the International Scientific Committee: Evaluation and treatment of urinary incontinence, pelvic organ prolapse, and fecal incontinence. Neurourology and urodynamics 29, 213-240 (2010).

7. Cerruto, M.A., et al. Insight into new potential targets for the treatment of overactive bladder and detrusor overactivity. Urologia internationalis 89, I-8 (2012).

8. Rosier, P.F.B., J.R. A prospective study to find evidence for the ICS 2002 terminology report; The ongoing debate in neurology and dynamics. Neurourology and urodynamics 25, 517-518 (2006).

9. Van Kerrebroeck, P.E. A treatment algorithm for the overactive bladder. BJU international 83 Suppl 2, 29-30 (1999).

10. Irwin, D.E., et al. Population-based survey of urinary incontinence, overactive bladder, and other lower urinary tract symptoms in five countries: results of the EPIC study. European urology 50, I306-13I4; discussion 1314-1305 (2006).

II. Abrams, P., et al. The standardisation of terminology of lower urinary tract function: report from the Standardisation Sub-committee of the International Continence Society. American journal of obstetrics and gynecology 187, I16-126 (2002).

12. Osman, N.I., et al. Detrusor underactivity and the underactive bladder: a new clinical entity? A review of current terminology, definitions, epidemiology, aetiology, and diagnosis. European urology 65, 389-398 (2014).

13. Feneley, R.C., Shepherd, A.M., Powell, P.H. \& Blannin, J. Urinary incontinence: prevalence and needs. British journal of urology 5I, 493-496 (1979).

14. Tincello, D.G., Rashid, T. \& Revicky, V. Emerging treatments for overactive bladder: clinical potential of botulinum toxins. Research and reports in urology 6, $51-57$ (2014).

15. Griffiths, D. Imaging bladder sensations. Neurourology and urodynamics 26, 899-903 (2007).

16. Fry C., B.A.F., Hussain M. Cell Biology, (Health Publication Ltd, Plymouth UK, 2005).

17. De Groat W.C., B.A.M., Yoshimura N. Incontinence. in Neuronal Control (ed. Abrams P. Cardozo L, K.S., Wein A.,) 363-422 (Health Publication Ltd., Plymouth UK, 2005). 
18. Yoshimura, N., et al. Neural mechanisms underlying lower urinary tract dysfunction. Korean journal of urology 55, 8I-90 (2014).

19. Drake, M.J. The integrative physiology of the bladder. Annals of the Royal College of Surgeons of England 89, 580-585 (2007).

20. Sadananda, P., Drake, M.J., Paton, J.F. \& Pickering, A.E. A functional analysis of the influence of beta3adrenoceptors on the rat micturition cycle. The Journal of pharmacology and experimental therapeutics 347 , 506-515 (2013).

21. de Groat, W.C. A neurologic basis for the overactive bladder. Urology 50, 36-52; discussion 53-36 (1997).

22. Wein A.J., C.C. Managing Overactive Bladder, (Springer Healthcare, London, 20II).

23. Fowler, C.J., Griffiths, D. \& de Groat, W.C. The neural control of micturition. Nature reviews. Neuroscience 9, 453-466 (2008).

24. Takahashi, O., et al. White matter lesions or Alzheimer's disease: which contributes more to overactive bladder and incontinence in elderly adults with dementia? Journal of the American Geriatrics Society 60, 2370-237I (2012).

25. Sherrington, C.S. Notes on the Arrangement of some Motor Fibres in the Lumbo-Sacral Plexus. The Journal of physiology 13, 621-772 617 (1892).

26. Sugaya, K. \& de Groat, W.C. Influence of temperature on activity of the isolated whole bladder preparation of neonatal and adult rats. American journal of physiology. Regulatory, integrative and comparative physiology 278, R238-246 (2000).

27. Drake, M.J., Harvey, I.J. \& Gillespie, J.I. Autonomous activity in the isolated guinea pig bladder. Experimental physiology 88, 19-30 (2003).

28. Lagou, M., Gillespie, J., Kirkwood, T., Harvey, I. \& Drake, M.J. Muscarinic stimulation of the mouse isolated whole bladder: physiological responses in young and ageing mice. Autonomic \& autacoid pharmacology 26, 253-260 (2006).

29. Gillespie, J.I. Modulation of autonomous contractile activity in the isolated whole bladder of the guinea pig. BJU international 93, 393-400 (2004).

30. Parsons, B.A., Drake, M.J., Gammie, A., Fry, C.H. \& Vahabi, B. The validation of a functional, isolated pig bladder model for physiological experimentation. Frontiers in pharmacology 3, 52 (2012).

31. Levin, R.M., Brendler, K. \& Wein, A.J. Comparative pharmacological response of an in vitro whole bladder preparation (rabbit) with response of isolated smooth muscle strips. The Journal of urology I30,377-38I (1983).

32. Maggi, C.A., et al. Contractile response of the human isolated urinary bladder to neurokinins: involvement of NK-2 receptors. European journal of pharmacology 145, 335-340 (1988).

33. van Waalwijk van Doorn, E.S., Remmers, A. \& Janknegt, R.A. Conventional and extramural ambulatory urodynamic testing of the lower urinary tract in female volunteers. The Journal of urology 147, 1319-1325; discussion 1326 (1992).

34. Robertson, A.S. Behaviour of the human bladder during natural filling: the Newcastle experience of ambulatory monitoring and conventional artificial filling cystometry. Scandinavian journal of urology and nephrology. Supplementum 20I, 19-24 (1999). 
Structural and Functional Aspects of Sensory-motor Interaction in the Urinary Bladder

35. Drake, M.J., et al. Partial outlet obstruction enhances modular autonomous activity in the isolated rat bladder. The Journal of urology 170, 276-279 (2003).

36. Gillespie, J.I., Harvey, I.J. \& Drake, M.J. Agonist- and nerve-induced phasic activity in the isolated whole bladder of the guinea pig: evidence for two types of bladder activity. Experimental physiology 88, 343-357 (2003).

37. Gillespie, J.I. The autonomous bladder: a view of the origin of bladder overactivity and sensory urge. BJU international 93, 478-483 (2004).

38. Gillespie, J.I. Noradrenaline inhibits autonomous activity in the isolated guinea pig bladder. BJU international 93, 40I-409 (2004).

39. Finney, S.M., Stewart, L.H. \& Gillespie, J.I. Volume-induced responses in the isolated bladder: evidence for excitatory and inhibitory elements. BJU international I02, II54-II6I (2008).

40. Streng, T., Hedlund, P., Talo, A., Andersson, K.E. \& Gillespie, J.I. Phasic non-micturition contractions in the bladder of the anaesthetized and awake rat. BJU international 97, I094-II0I (2006).

4I. Coolsaet, B.L., Van Duyl, W.A., Van Os-Bossagh, P. \& De Bakker, H.V. New concepts in relation to urge and detrusor activity. Neurourology and urodynamics 12, 463-47I (1993).

42. Birder, L.A., et al. Alterations in $\mathrm{P} 2 \mathrm{X}$ and $\mathrm{P} 2 \mathrm{Y}$ purinergic receptor expression in urinary bladder from normal cats and cats with interstitial cystitis. American journal of physiology 287, FI084-I09I (2004).

43. Birder, L.A., et al. Beta-adrenoceptor agonists stimulate endothelial nitric oxide synthase in rat urinary bladder urothelial cells. J Neurosci 22, 8063-8070 (2002).

44. Beckel, J.M., Kanai, A., Lee, S.J., de Groat, W.C. \& Birder, L.A. Expression of functional nicotinic acetylcholine receptors in rat urinary bladder epithelial cells. American journal of physiology 290, FI03-IIO (2006).

45. D’Andrea, M.R., Saban, M.R., Nguyen, N.B., Andrade-Gordon, P. \& Saban, R. Expression of proteaseactivated receptor-I, $-2,-3$, and -4 in control and experimentally inflamed mouse bladder. The American journal of pathology 162, 907-923 (2003).

46. Du, S., et al. Amiloride-sensitive ion channels in urinary bladder epithelium involved in mechanosensory transduction by modulating stretch-evoked adenosine triphosphate release. Urology 69, 590-595 (2007).

47. Yoshimura, N., et al. Therapeutic receptor targets for lower urinary tract dysfunction. NaunynSchmiedeberg's archives of pharmacology 377, 437-448 (2008).

48. Streng, T., et al. Distribution and function of the hydrogen sulfide-sensitive TRPAI ion channel in rat urinary bladder. European urology 53, 39I-399 (2008).

49. Stein, R.J., et al. Cool (TRPM8) and hot (TRPVI) receptors in the bladder and male genital tract. The Journal of urology 172, II75-1I78 (2004).

50. Birder, L., et al. Activation of urothelial transient receptor potential vanilloid 4 by 4 alpha-phorbol 12,13-didecanoate contributes to altered bladder reflexes in the rat. The Journal of pharmacology and experimental therapeutics 323, 227-235 (2007).

51. Yoshida, M., et al. Non-neuronal cholinergic system in human bladder urothelium. Urology 67,425-430 (2006).

52. Birder, L.A., et al. How does the urothelium affect bladder function in health and disease? ICI-RS $201 \mathrm{I}$. Neurourology and urodynamics 31, 293-299 (2012). 
53. Narumiya, S., Sugimoto, Y. \& Ushikubi, F. Prostanoid receptors: structures, properties, and functions. Physiological reviews 79, I193-1226 (1999).

54. Gilmore, N.J. \& Vane, J.R. Hormones released into the circulation when the urinary bladder of the anaesthetized dog is distended. Clinical science 4I, 69-83 (1971).

55. Poggesi, L., et al. The role of prostaglandins in the maintenance of the tone of the rabbit urinary bladder. Invest Urol 17, 454-458 (1980).

56. Rahnama'i, M.S., et al. Prostaglandin receptor EPI and EP2 site in guinea pig bladder urothelium and lamina propria. The Journal of urology 183, 124I-1247 (2010).

57. Rahnama'i, M.S., Biallosterski, B.T., de Wachter, S.G., Van Kerrebroeck, P.E. \& van Koeveringe, G.A. The distribution of the prostaglandin E receptor type 2 (EP2) in the detrusor of the guinea pig. Prostaglandins \& other lipid mediators 99, 107-115 (2012).

58. Rahnama'i, M.S., et al. The relationship between prostaglandin E receptor I and cyclooxygenase I expression in guinea pig bladder interstitial cells: proposition of a signal propagation system. The Journal of urology 185, 315-322 (2011).

59. Rahnama'i, M.S., et al. The relationship between prostaglandin E receptor I and cyclooxygenase I expression in guinea pig bladder interstitial cells:proposition of a signal propagation system. The Journal of urology I85,3I5-322.

60. Ishizuka, O., Mattiasson, A. \& Andersson, K.E. Prostaglandin E2-induced bladder hyperactivity in normal, conscious rats: involvement of tachykinins? J Urol I53, 2034-2038 (1995).

6I. de Groat, W.C. \& Yoshimura, N. Pharmacology of the lower urinary tract. Annual review of pharmacology and toxicology 4I, 69I-72I (200I).

62. Johns, A. \& Paton, D.M. Effect of indomethacin on atropine-resistant transmission in rabbit and monkey urinary bladder: evidence for involvement of prostaglandins in transmission. Prostaglandins 13, 245-254 (1977).

63. Burnstock, G., Cocks, T., Crowe, R. \& Kasakov, L. Purinergic innervation of the guinea-pig urinary bladder. BrJ Pharmacol 63, I25-I38 (1978).

64. Borda, E., Contreras-Ortiz, N., Gutnisky, R. \& Gimeno, M.F. In vitro effect of acetylcholine and bethanechol on the contractions of the human detrusor muscle. Influence of prostaglandins. Archives internationales de pharmacodynamie et de therapie 259, 31 -39 (1982).

65. Andersson, K.E. \& Sjogren, C. Aspects on the physiology and pharmacology of the bladder and urethra. Progress in neurobiology 19, 71-89 (1982).

66. Shea, V.K., Cai, R., Crepps, B., Mason, J.L. \& Perl, E.R. Sensory fibers of the pelvic nerve innervating the Rat's urinary bladder. Journal of neurophysiology 84, 1924-1933 (2000).

67. Gosling, J.A. \& Dixon, J.S. Sensory nerves in the mammalian urinary tract. An evaluation using light and electron microscopy. J Anat II7, 133-144 (1974).

68. Andersson, K.E. Bladder activation: afferent mechanisms. Urology 59, 43-50 (2002).

69. Grol, S., van Koeveringe, G.A., de Vente, J., van Kerrebroeck, P.E. \& Gillespie, J.I. Regional differences in sensory innervation and suburothelial interstitial cells in the bladder neck and urethra. BJU Int (2008).

70. Gillespie, J.I., Markerink-van Ittersum, M. \& de Vente, J. Sensory collaterals, intramural ganglia and motor nerves in the guinea-pig bladder: evidence for intramural neural circuits. Cell Tissue Res 325, 33-45 (2006). 
Structural and Functional Aspects of Sensory-motor Interaction in the Urinary Bladder

7I. Gillespie, J.I., van Koeveringe, G.A., de Wachter, S.G. \& de Vente, J. On the origins of the sensory output from the bladder: the concept of afferent noise. BJU Int 103, 1324-1333 (2009).

72. Xu, L. \& Gebhart, G.F. Characterization of mouse lumbar splanchnic and pelvic nerve urinary bladder mechanosensory afferents. J Neurophysiol 99, 244-253 (2008).

73. Lagou, M., et al. Location of interstitial cells and neurotransmitters in the mouse bladder. BJU international 97, 1332-1337 (2006).

74. Ouslander,J.G. Management of overactive bladder. The New England journal of medicine 350, 786-799 (2004).

75. Yoshimura, N. \& Chancellor, M.B. Current and future pharmacological treatment for overactive bladder. JUrol 168, 1897-1913 (2002).

76. Irwin, D.E., Milsom, I., Kopp, Z., Abrams, P. \& Cardozo, L. Impact of overactive bladder symptoms on employment, social interactions and emotional well-being in six European countries. BJU international 97 , 96-100 (2006).

77. Homma, Y., Yamaguchi, O., Hayashi, K. \& Neurogenic Bladder Society, C. An epidemiological survey of overactive bladder symptoms in Japan. BJU international 96, I3|4-1318 (2005).

78. Teloken, C., et al. Overactive bladder: prevalence and implications in Brazil. European urology 49, 1087 1092 (2006).

79. Milsom, l., et al. How widespread are the symptoms of an overactive bladder and how are they managed? A population-based prevalence study. BJU international 87, 760-766 (200I).

80. Coyne, K.S., et al. The prevalence of lower urinary tract symptoms (LUTS) and overactive bladder (OAB) by racial/ethnic group and age: results from OAB-POLL. Neurourology and urodynamics 32, 230-237 (20I3).

8I. Hu, T.W. \& Wagner, T.H. Economic considerations in overactive bladder. The American journal of managed care 6, S59I-598 (2000).

82. Kelleher, C., Friel, S., Nolan, G. \& Forbes, B. Effect of social variation on the Irish diet. The Proceedings of the Nutrition Society 6I, 527-536 (2002).

83. Turner, D.A., et al. The cost of clinically significant urinary storage symptoms for community dwelling adults in the UK. BJU international 93, 1246-1252 (2004).

84. Klotz, T., Bruggenjurgen, B., Burkart, M. \& Resch, A. The economic costs of overactive bladder in Germany. European urology 5I, 1654-1662; discussion I662-1653 (2007).

85. Lee, S.H., Cho, S.T., Na, H.R., Ko, S.B. \& Park, M.H. Urinary incontinence in patients with Alzheimer's disease: Relationship between symptom status and urodynamic diagnoses. International journal of urology: official journal of the Japanese Urological Association 21, 683-687 (2014).

86. Mattson, M.P. Pathways towards and away from Alzheimer's disease. Nature 430, 631-639 (2004).

87. Ransmayr, G.N., et al. Lower urinary tract symptoms in dementia with Lewy bodies, Parkinson disease, and Alzheimer disease. Neurology 70, 299-303 (2008).

88. Han, D. \& Wang, Y. Urinary Incontinence in Dementia Incont Pelvic Floor Dysfunct 2, 63-66 (2008).

89. Jirovec, M.M. \& Wells, T.J. Urinary incontinence in nursing home residents with dementia: the mobilitycognition paradigm. Appl Nurs Res 3, II2-117 (1990).

90. Serra, L., et al. Relationship between cognitive impairment and behavioural disturbances in Alzheimer's disease patients. Behavioural neurology 23, 123-130. 
91. Fan, Y.H., Lin, A.T., Wu, H.M., Hong, C.J. \& Chen, K.K. Psychological profile of female patients with dysfunctional voiding. Urology 7I, 625-629 (2008).

92. Sugiyama, T., et al. Urinary incontinence in senile dementia of the Alzheimer type (SDAT). Int J Urol I, 337-340 (1994).

93. Biallosterski, B.T., et al. Changes in bladder innervation in a mouse model of Alzheimer's disease. Journal of chemical neuroanatomy 39, 204-210.

94. Mostwin, J.L., Karim, O.M., van Koeveringe, G. \& Brooks, E.L. The guinea pig as a model of gradual urethral obstruction. The Journal of urology 145, 854-858 (1991).

95. Klevmark, B. Motility of the urinary bladder in cats during filling at physiological rates. II. Effects of extrinsic bladder denervation on intramural tension and on intravesical pressure patterns. Acta physiologica Scandinavica I0I, I76-184 (1977).

96. Vaughan, C.W. \& Satchell, P.M. Role of sympathetic innervation in the feline continence process under natural filling conditions. Journal of neurophysiology 68, 1842-I849 (1992).

97. Gillespie, J.I. A developing view of the origins of urgency: the importance of animal models. BJU international 96 Suppl I, 22-28 (2005).

98. de Jongh, R., et al. The localisation of cyclo-oxygenase immuno-reactivity (COXI-IR) to the urothelium and to interstitial cells in the bladder wall. Journal of cellular and molecular medicine (2008).

99. de Jongh, R., et al. The effects of exogenous prostaglandins and the identification of constitutive cyclooxygenase I and II immunoreactivity in the normal guinea pig bladder. BJU international I00, 419-429 (2007).

100. Kohler, C., Ebert, U., Baumann, K. \& Schroder, H. Alzheimer's disease-like neuropathology of genetargeted APP-SLXPSImut mice expressing the amyloid precursor protein at endogenous levels. Neurobiology of disease 20, 528-540 (2005).

I0I. Duff, K., et al. Increased amyloid-beta42(43) in brains of mice expressing mutant presenilin I. Nature 383, 710-713 (1996).

102. Vanmierlo, T., et al. Alterations in Brain Cholesterol Metabolism in the APPSLxPSImut mouse, a Model for Alzheimer's Disease. J Alzheimers Dis (2009).

103. Vanmierlo, $T$., et al. Liver $X$ receptor activation restores memory in aged $A D$ mice without reducing amyloid. Neurobiol Aging (2009).

104. Rasmusson, D.X., et al. Accuracy of clinical diagnosis of Alzheimer disease and clinical features of patients with non-Alzheimer disease neuropathology. Alzheimer Dis Assoc Disord 10, 180-188 (1996).

105. Hedlund, P. Nitric oxide/cGMP-mediated effects in the outflow region of the lower urinary tract--is there a basis for pharmacological targeting of cGMP? World J Urol 23, 362-367 (2005).

106. Wood, R., Eichel, L., Messing, E.M. \& Schwarz, E. Automated noninvasive measurement of cyclophosphamide-induced changes in murine voiding frequency and volume. The Journal of urology 165 , 653-659 (200I).

107. Stechman, M.J., et al. Establishing normal plasma and 24-hour urinary biochemistry ranges in $\mathrm{C} 3 \mathrm{H}$, $\mathrm{BALB} / \mathrm{c}$ and $\mathrm{C} 57 \mathrm{BL} / 6 \mathrm{~J}$ mice following acclimatization in metabolic cages. Laboratory animals 44, 218-225. 

Chapter 2

\section{The Distribution of the Prostaglandin E Receptor Type 2 (EP2) in the Detrusor of the Guinea Pig}

Mohammad S. Rahnama'i, Bart T. Biallosterski, Stefan G.G. de Wachter, Philip E.V. Van Kerrebroeck and Gommert A. van Koeveringe

PMID: 2296043I

Prostaglandins \& Other Lipid Mediators 2012 
Structural and Functional Aspects of Sensory-motor Interaction in the Urinary Bladder

\begin{abstract}
Purpose

To explore the distribution of prostaglandin E receptor type 2 (EP2) in the bladder muscle layers and its spatial relationship to cyclo-oxygenase type I (COXI).

\section{Materials and methods}

Twelve male guinea pigs were killed by cervical dislocation, the bladders removed and fixed in $4 \%$ paraformaldehyde in PBS. Frozen sections of $10 \mu \mathrm{m}$ were cut and stained with antibodies to EP2, COXI and vimentin.
\end{abstract}

\title{
Results
}

EP2 receptor immunoreactivity is located on the smooth muscle cells as well as on vimentin positive surface muscle and intramuscular interstitial cells. EP2 expression on interstitial cells is highly

localized. Discrete regions of intense staining were observed on the interstitial cell processes. COXI is expressed in the muscle interstitial cells and was found to be located on discrete regions of the cell and cell processes. Double staining with EP2 and COXI suggests that the regions of a cell expressing EP2 are different from those expressing COXI.

\section{Conclusions}

The presence of COXI, prostaglandin E receptor type 2 (EP2) immune-reactivity in the network of interstitial cells suggests a role of this network in the propagation of signals. Due to a cAMP coupling of the EP2 receptor in many other tissues and a lower dissociation constant of EP2, it is suggested that a rise in PG levels may gradually push the balance from a relaxant EP2 effect towards a contractile effect. Hence, PG could have a modulatory role on the non-voiding bladder contractions by changing the threshold level for excitability of the interstitial cell network. 
The Distribution of the Prostaglandin E Receptor Type 2 (EP2) in the Detrusor of the Guinea Pig

\section{Introduction}

It is now generally accepted that prostaglandin (PG) plays a role in the control of mammalian urinary bladder motility ${ }^{1,2}$. In the bladder, both the lamina propria and the muscle layers can produce Prostaglandins ${ }^{3-6}$. Prostaglandin E2 $\left(\mathrm{PGE}_{2}\right)$ is known to be the predominant $\mathrm{PG}$ in the urinary bladder of most mammals ${ }^{7}$ and the receptors for PGE $_{2}$ are classified into four types: EPI, EP2, EP3 and EP4 ${ }^{8}$. $P G E_{2}$ is an agonist at EP receptors $1-4$, all G-protein coupled, which mediate its physiological effects ${ }^{9}$. $\mathrm{PGE}_{2}$ is the main $\mathrm{PG}$ suggested to be involved in the pathophysiology of detrusor overactivity (DO) and overactive bladder syndrome (OAB) ${ }^{9,10}$. This involvement is based on three observations. Firstly, the ability of $\mathrm{PGE}_{2}$ infused into the bladder to induce DO in humans and animals. Secondly, the increased $\mathrm{PGE}_{2}$ production in DO models and thirdly, the high concentrations of $\mathrm{PGE}_{2}$ that have been detected in the urine of patients with $\mathrm{OAB}{ }^{9}$. It has also been shown that upon stretch, $\mathrm{PGE}_{2}$ is released by the urothelium " and that this release is regulated by a complex interaction of signals through ATP and NO !.

the literature contains substantial data that the wall of the mammalian urinary bladder contains several sub-populations of cells in the interstitial space, called interstitial cells (ICs) after the interstitial cells of Cajal in the gut ${ }^{12}$. These interstitial cells are located in the lamina propria and within the detrusor and have been suggested to form a network called the interstitial cell network ${ }^{3,13-15}$. The expression of prostaglandin $E$ receptor type I (EPI) in the guinea pig muscle interstitial cell network has been studied recently ${ }^{3}$.

Moreover, it has been shown that the cyclo-oxygenase I (COXI) enzymes are located within specific cell types within the lamina propria of the guinea pig bladder ${ }^{16}$. In addition, COXI is found in the basal layers of the urothelium and associated with the distributed network of lamina propria interstitial cells ${ }^{16}$. It has also been suggested that the arrangement of EPI and COXI might

have the potential to facilitate the propagation of signals in the interstitial cell network ${ }^{3}$. Such a signalling system is thought to have a role in coordinating events in the normal bladder ${ }^{17}$ and might be defective in bladder pathology ${ }^{13}$. It might also have a function in facilitating the global coordinated changes associated with bladder wall remodelling ${ }^{3,18}$. PG receptors EPI and EP2 have been shown to be expressed in the ICs in the lamina propria indicating that this part of the bladder wall can respond to $\mathrm{PGE}_{2}{ }^{4}$. There is hardly any literature about the exact role of the EP2 receptor in the bladder. However, it is known that the normal guinea pig urothelium does express EP2 receptors ${ }^{4}$ and that the combined EPI/EP2 receptor antagonist $\mathrm{AH} 6809$ decreases detrusor contraction in isolated human bladder experiments ${ }^{19}$. Unlike EPI ${ }^{3}$, the cellular localization and the expression of EP2 receptor in the muscle layers of the bladder have not been studied before. In the present study, we have used antibodies to EP2 in order to study the distribution of this receptor in the muscle layers of the guinea pig urinary bladder. Our results are discussed in terms of possible physiological mechanisms, which might be occurring in this region of the bladder wall. 
Structural and Functional Aspects of Sensory-motor Interaction in the Urinary Bladder

\section{Materials and methods}

Guinea pigs (12 male, weight range 260-300 g) were killed by cervical dislocation. All procedures were carried out in agreement with the guidelines of the Maastricht University Ethical Committee. The tissue workup of the lateral wall of the guinea pig bladders as well as freezing and the cutting of the sections are as described in our previous paper ${ }^{4}$.

\section{Tissue preparation}

The bladder, including the proximal urethra, was removed from each animal and placed in ice-cold Krebs solution composed of I2I.I mM NaCl, I.87 mM KCl, I.2 mM CaCl2, I.I5 mM MgSO4, $25 \mathrm{mM}$ $\mathrm{NaHCO} 3, \mathrm{I} .17 \mathrm{mM} \mathrm{KH} 2 \mathrm{PO} 4$ and II.0 mM glucose bubbled with 5\% CO2 and 95\% O2 (pH 7.4). Each bladder was divided into a ventral piece and a dorsal piece, while maintained in Krebs solution. Then, the bladder pieces were immersed in ice-cold fixative solution of $4 \%$ freshly prepared depolymerized paraformaldehyde for $120 \mathrm{~min}$ at $4{ }^{\circ} \mathrm{C}$. Tissues were fixed in 3 steps of 2 overnight and I daytime incubation at $4{ }^{\circ} \mathrm{C}$ in $0.1 \mathrm{M}$ phosphate buffer with $10 \%, 20 \%$ and $30 \%$ sucrose, respectively.

On day 3 the tissues were placed in Tissue-Tek $®$ OCTTM compound to form a single block, snap frozen in isopentane and cooled in liquid nitrogen. Cryostat sections $(10 \mu \mathrm{m})$ were cut such that each section was perpendicular to the urothelial surface. Sections were thawed on chrome alum-gelatin coated slides and processed for immunocytochemistry.

\section{Characterization of the EP2 antibody}

In order to gain insight in the specificity of our antibody, we conducted a pre-absorption test and characterized the antibodies by Western blotting as described in our previous paper [4]. In short, preabsorption was done by overnight incubation of the anti-EP 2 antibody (I:I00 by Cayman Chemical, Catalogue No. 301740) with or without $10 \mu \mathrm{g} / \mathrm{ml}$ of the peptide against which the antibody was raised. Thereafter the antibody solution or antibody plus peptide solution was applied to the sections. Bladder homogenate of guinea pig bladders $(N=2)$ was prepared for Western blotting. Bladders were cut into squares of approximately $1 \mathrm{~mm} 2$ and homogenized using an UltraTurrax homogenizer (Janke \& Kunkel, Ika Labortechnik, Staufen, Germany) at $4{ }^{\circ} \mathrm{C}$ in IX radio-immunoprecipitation assay buffer (I\% Triton-X-100, $137 \mathrm{mM} \mathrm{NaCl}, 20 \mathrm{mM}$ Tris- $\mathrm{HCl}, 2 \mathrm{mM}$ EDTA, $10 \%$ glycerol, I mM sodium orthovanadate, $10 \mathrm{mM} \mathrm{NaF}$, I× protease inhibitor cocktails, I mM phenylmethylsulphonyl fluoride). Subsequently the homogenates were centrifuged at $13,000 \times \mathrm{g}$ for $20 \mathrm{~min}$ at $4^{\circ} \mathrm{C}$. The pellet was discarded and the supernatant was stored at $-80^{\circ} \mathrm{C}$. Protein measurements were done using bovine albumin serum dilutions with the Biorad protein measurement system (Bio-Rad Laboratories, Inc., Hertfordshire, CA, USA) according to the manufacturer's instructions. The blot analysis was performed under reducing conditions following standard procedures and using the Odyssey infrared imaging system (Li-cor Biosciences, USA). Primary antibody used for Western blotting was rabbit anti-EP2 antibody (Cayman Chemicals) used in a dilution of I:I00. The staining was also confirmed by a blocking 
The Distribution of the Prostaglandin E Receptor Type 2 (EP2) in the Detrusor of the Guinea Pig

peptide on the Western blot using pre-absorption with anti-EP2 antibody in a dilution of I:100 (Cayman Catalogue Nos. 301750 and 301740). The secondary antibody used was donkey antirabbit IRdye800 (Rockland, 6II-732-I27).

\section{Immunohistochemistry}

Sections were dried for $20 \mathrm{~min}$ at room temperature followed by three washes with Tris-buffered saline (TBS; $\mathrm{pH} 7.6$ ), and thereafter incubated overnight with primary antibodies at $4{ }^{\circ} \mathrm{C}$. To visualize the prostaglandin receptor EP2, we used a polyclonal antibody rabbit anti-EP2 (Cayman Chemical, Catalogue No. 101750) which was diluted I:I00. The COXI enzyme was visualized using an optimized concentration of goat polyclonal antibody to COXI (I:2000; Santa Cruz Biotech, Catalogue No. sc-1752). Furthermore, a mouse antibody against vimentin (Sigma-Aldrich, Catalogue No. V 5355) was used at a dilution of I:5000. The rabbit primary antibody was visualized using Alexa Fluor 488 donkey anti-rabbit $\operatorname{lgG}(\mathrm{H}+\mathrm{L})$ conjugate (Molecular Probes), diluted I:100 in TBS-T. The mouse primary antibody was visualized with Alexa Fluor 594 donkey antimouse IgG conjugate (Molecular Probes), diluted I:I00. The goat primary antibody was visualized with Alexa Fluor 594 donkey antigoat lgG conjugate (Molecular Probes). Sections were incubated with the secondary antibodies for $60 \mathrm{~min}$ at room temperature in the dark. After three wash steps the sections were incubated at room temperature for 10 min with a nuclei marker, HOECHST 33342 (Sigma, Zwijndrecht, The Netherlands) and mounted with TBS-glycerol. All stainings were done in all the animals and duplicated and repeated on at least 2 separate days. All results were confirmed with negative control stainings, without application of the primary antibody. Observations were accumulated from the different slides and from the different bladder specimens of all 12 animals. A representative section of all animal bladders was studied.

\section{Microscopy}

The majority of the sections was analysed and photographed using an Olympus AX70 microscope using a 10x, 20x and 40x objective. For the detection of Alexa 488 fluorescence, a narrow bandpass MNIBA-filter and for Alexa 594 a narrow excitation band U-M4I007A filter was used (both from Chroma Technologies). Confocal laser scanning microscopy fluorescent imaging was done on the samples using a Bio-Rad MRC600 confocal microscope (Bio-Rad Laboratories Ltd., Hemel Hempstead, UK) equipped with an air-cooled Argon-Krypton mixed gas laser and mounted onto an Axiophotemicroscope (Zeiss), using oil-immersion objectives (40, NA1/43DI.3 or 63, NA1/43DI.4). The laser-scanning microscope was used in dual wavelength excitation at 488 and $568 \mathrm{~nm}$.

Optical sections were recorded in the Kalman filtering mode using 4-8 scans for each picture. Z-series were generated by collecting a stack consisting of optical sections using a step size between 0.18 and $0.46 \mathrm{~mm}$ in the $\mathrm{z}$-direction. 


\section{Results}

Figure I shows the characterization of the EP2 antibody used in this study. This characterization was described in our previous paper ${ }^{4}$. There were two approaches chosen to confirm that this is specific staining. Firstly, the antibody was pre-incubated with the peptide to which it was raised (blocking peptide) and the antibodies specific reactive site was eliminated. Where this was done, all of the observed staining was removed (panel A). In order to see the tissue morphology, an enhanced (overexposed) image is shown in the inset. Panel $B$ shows a Western blot of bladder protein exposed EP2 antibody (middle lane). A single protein band of the predicted molecular weight of the EP2 protein was seen. This band was also removed by pre-absorbing the antibody with the blocking peptide. Thus, this antibody for EP2 can be used with a high degree of certainty that they will detect specifically the EP2 receptors in the guinea pig bladder.

The EP2 receptor is present on both the detrusor smooth muscle cells as well as on vimentin positive muscle interstitial cells (Figure 2). The vast majority of interstitial cells clearly express EP2 receptors. More detail about the EP2 expression is shown in Figures 2, 5 and 6 where it can be noted that the EP2 expression is not equal throughout the cell and appears to be localized in discrete regions.

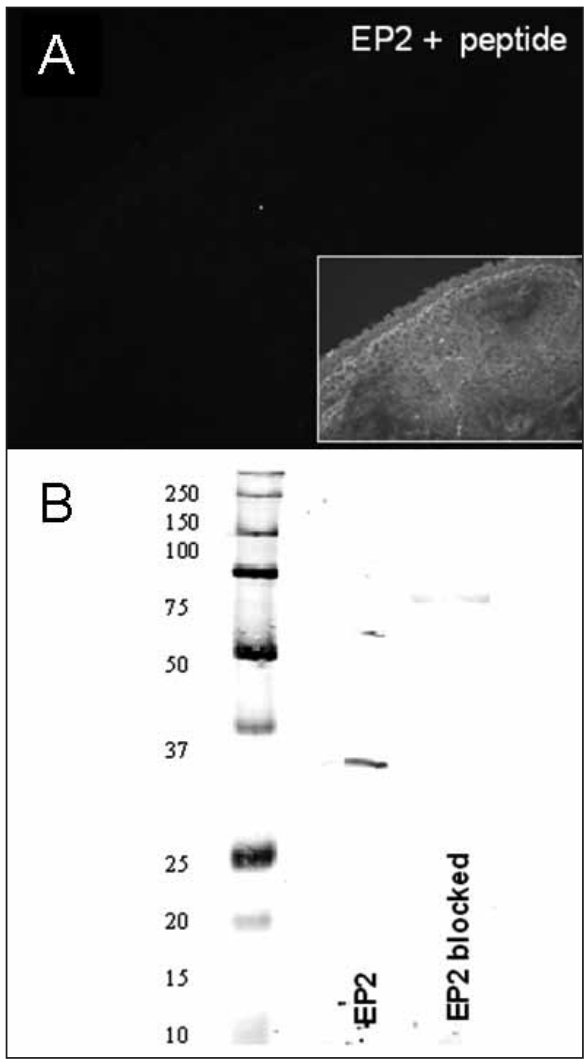

Figure I. Characterization of the EP2 antibody. Panel A illustrates the staining observed with the same concentration of antibody as used in all the stainings of other figures, but with pre-incubation with the blocking peptide to which the antibody was raised. (The inset shows an enhanced image showing the section morphology.) Calibration bar: $10 \mu \mathrm{m}$. Panel B shows a characterization of the antibodies using Western blotting. Three lanes are shown: the molecular weight calibration bands, the native protein stained with the EP2 antibody and the native protein but exposed to antibody plus appropriate blocking peptide. 


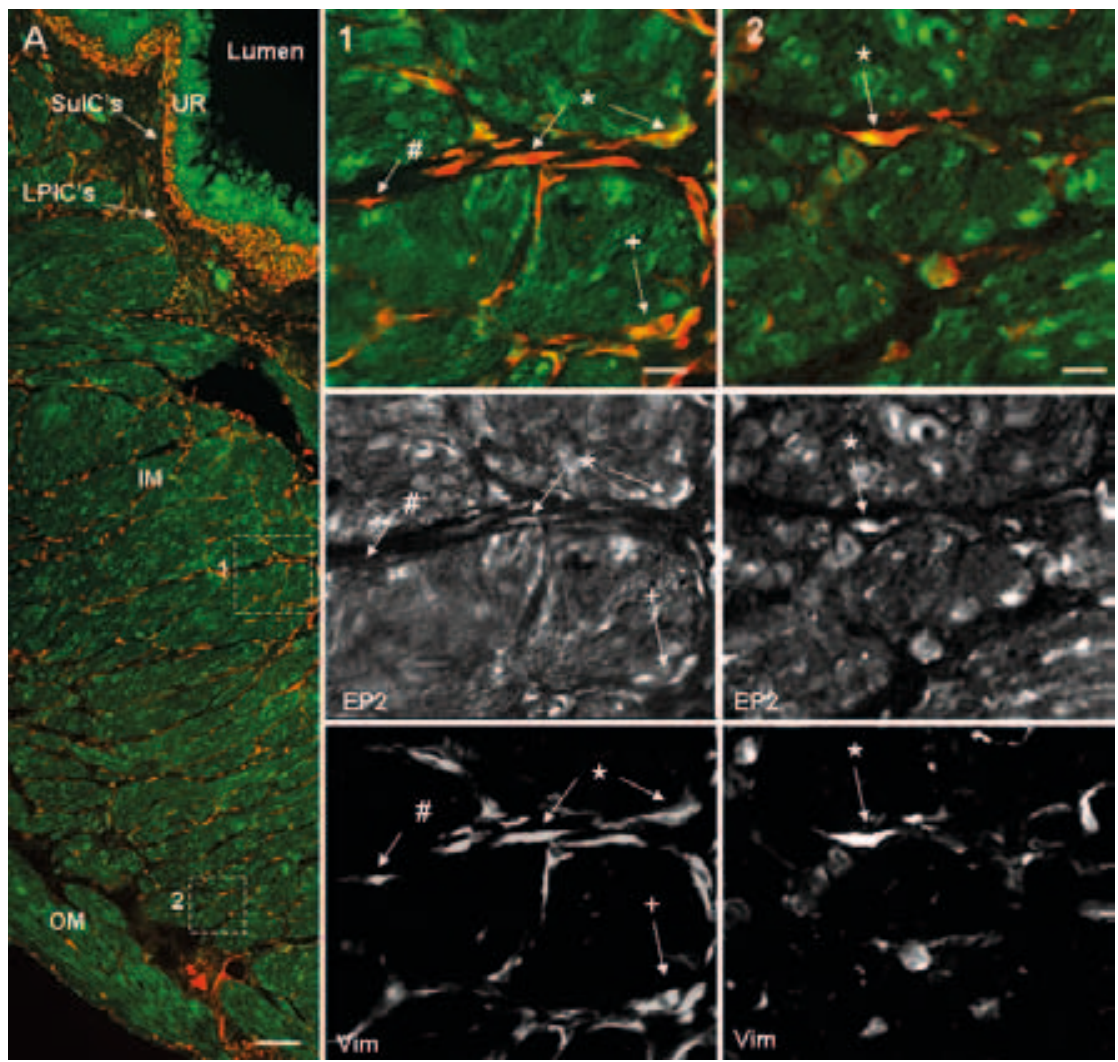

Figure 2. The distribution of EP2 and vimentin in the inner- and outer-muscle layers of the lateral wall of the guinea pig bladder. Panels show an image of the guinea pig bladder stained with the antibody for EP2 (green) and vimentin (red). In panel A, a low magnification image of a full thickness guinea pig bladder section is shown. The bladder lumen, the urothelium (UR), sub-urothelial interstitial cells (SuIC's), the lamina propria interstitial cells (LPICs), inner muscle (IM) and outer muscle layer (OM) are marked. In the right panels, regions of interest of the inner (I) and the outer muscle layer (2) are shown in a larger magnification. Interstitial cells co-expressing vimentin and EP2 receptor are marked with * and those vimentin positive interstitial cells not expressing EP2 are marked with '\#'. Calibration bars: $100 \mu \mathrm{m}$ in A and $20 \mu \mathrm{m}$ in the insets $\mathrm{I}$ and 2.

In figure 3, vimentin positive (red) processes of intramuscular interstitial cells (IM-ICs) are clearly visible. These fibres also express EP2 (green). The EP2 staining on the muscle cells is less intense and has a punctuate appearance. 


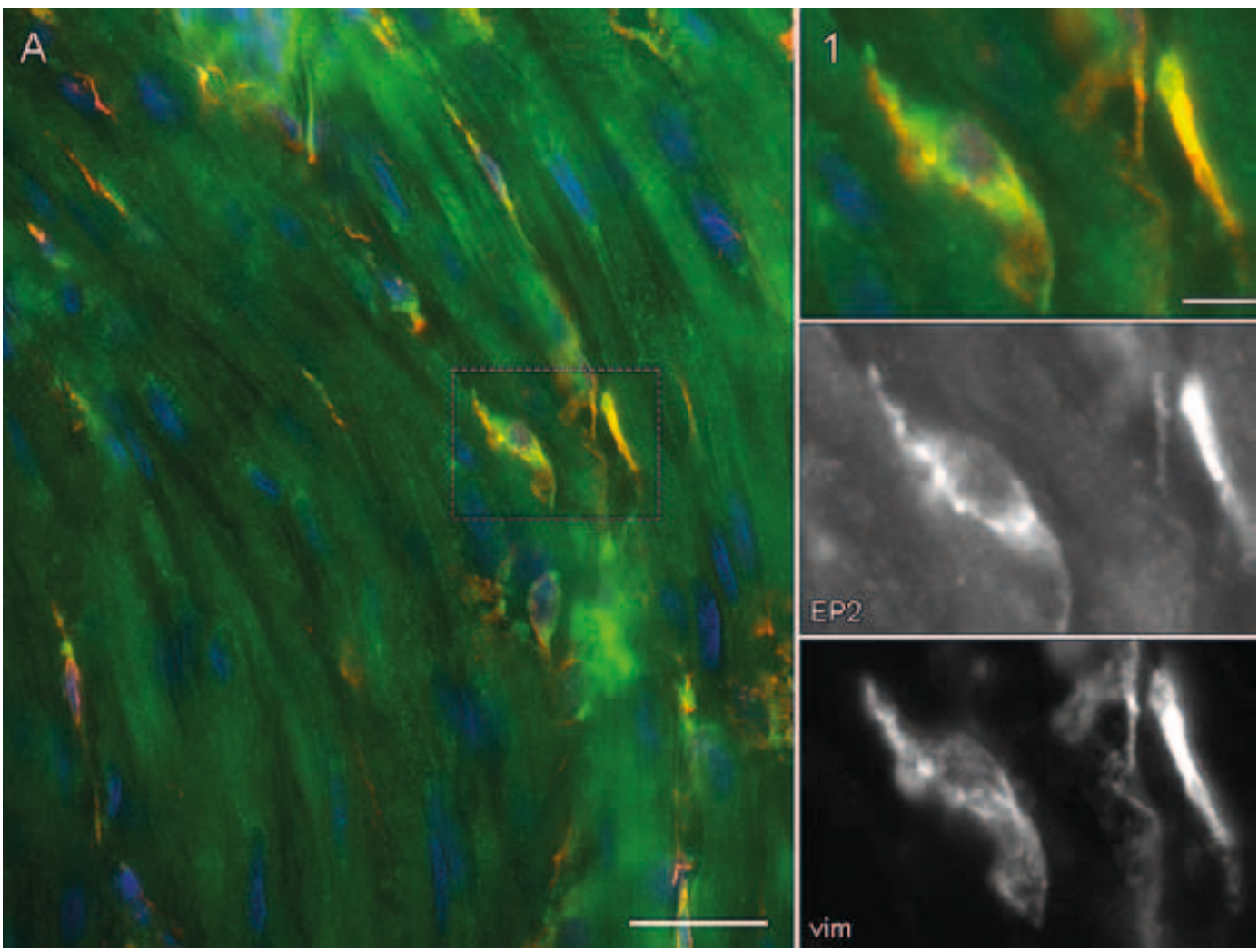

Figure 3. The distribution of EP2 and vimentin in the inner muscle layer of the lateral wall of the guinea pig bladder. An image taken from a confocal stack of the inner muscle layer of the guinea pig bladder stained with the antibody for EP2 (green) and, vimentin (red) is shown. The nuclei of the IM-ICs are visualized by a HOECHST nuclei staining (blue). A region of interest is enlarged in inset I. The black and white insets show the individual EP2 and vimentin staining, respectively. Calibration bar: $40 \mu \mathrm{m}$ in $\mathrm{A}$ and $10 \mu \mathrm{m}$.

The EP2 expression in the outer muscle layer is shown in Figure 4. In the middle of the muscle bundles, IM-ICs can be seen in which the EP2 (green) and vimentin (red) co-localization is visible by the yellow colour. Thus, the vimentin positive interstitial cells seem to express the EP 2 receptor. In this outer muscle region, the muscle is positive for EP2 with a less intense punctuate staining. On the upper edge of the panel, muscle coat interstitial cells are visible. Black and white panels on the right show clearly that only some of the muscle coat vimentin positive interstitial cells express EP2. Distinct groups of vimentin positive interstitial cells are seen between the muscle bundles. 


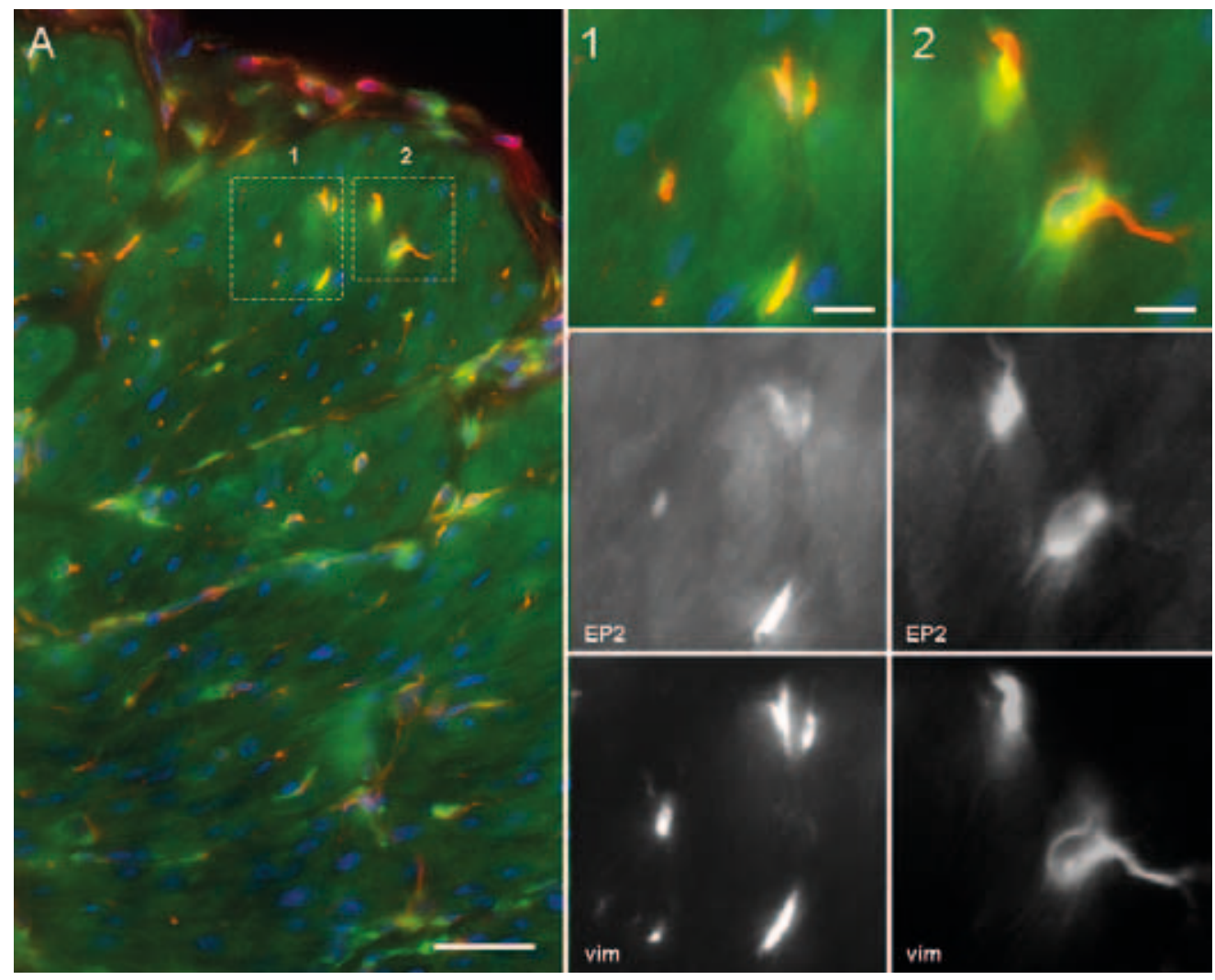

Figure 4. The distribution of EP2 and vimentin in the outer muscle layer of the lateral wall of

the guinea pig bladder. An image taken from a confocal stack of the outer muscle layer of the guinea pig bladder stained with the antibody for EP2 (green) and vimentin (red) is shown. The nuclei of the IM-ICs are visualized by a HOECHST nuclei staining (blue). Two regions of interest from panel A are enlarged as panels I and 2. The black and white insets show the individual EP2 and vimentin staining, respectively. Calibration bars: $100 \mu \mathrm{m}$ in A and I0 $\mu \mathrm{m}$ in I and 2. (For interpretation of the references to colour in this figure legend, the reader is referred to the web version of the article.)

In figure 5, a number of ICs are shown in the vicinity of each other. The fibres of these cells are noted as red fibres (vimentin positive) running through and in between the muscle bundles. Many of these ICs co-express vimentin and EP2 causing the yellow co-staining. In figure 5 the expression of EP2 in an IM-IC in the lateral wall is shown. The processes of these cells are visible as vimentin positive fibres (red) running on the surface of the muscle bundles. Spots of EP2 expression (green) are also visible on these fibres. Moreover, the punctuate expression of EP2 on the muscle bundles can be seen in Figure 6. 


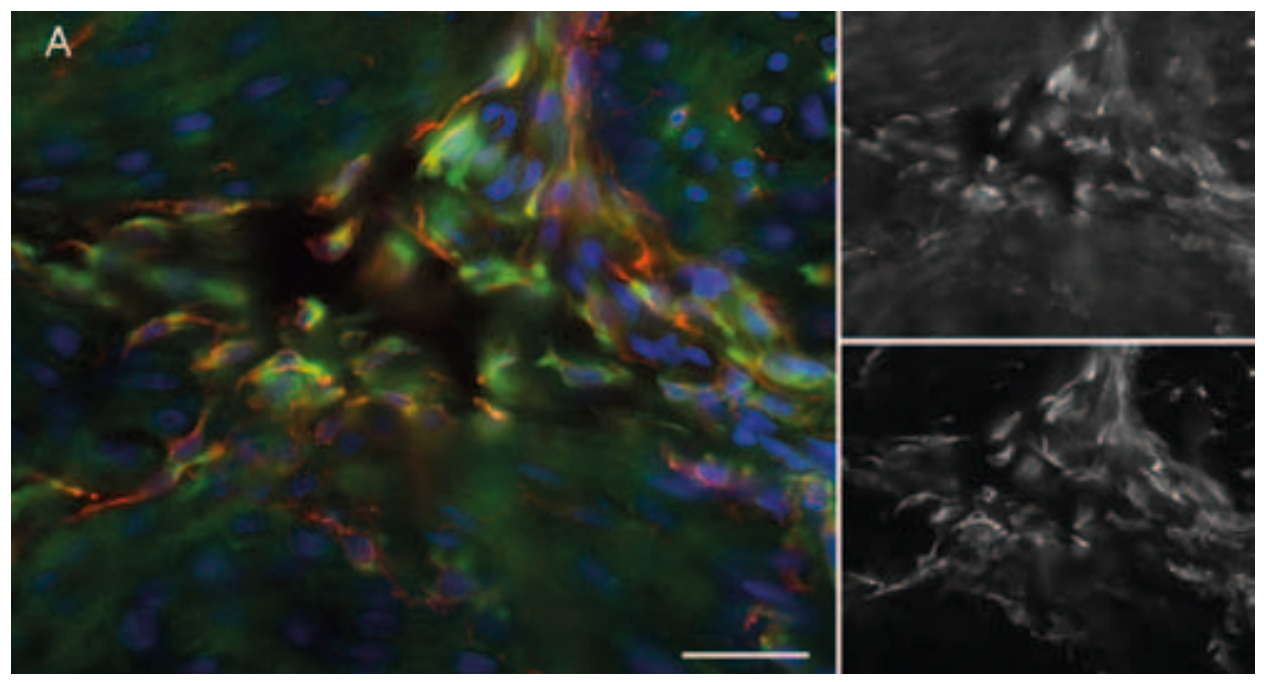

Figure 5. The expression of EP2 in a group of nearby intramuscular interstitial cells in the lateral wall of the guinea pig bladder. An image of the outer muscle layer of the guinea pig bladder stained with the antibody for EP2 (green) and vimentin (red) is shown. The nuclei of the cells are visualized by a HOECHST nuclei staining (blue). The black and white insets show the individual EP2 and vimentin staining, respectively. Calibration bars: $20 \mu \mathrm{m}$.

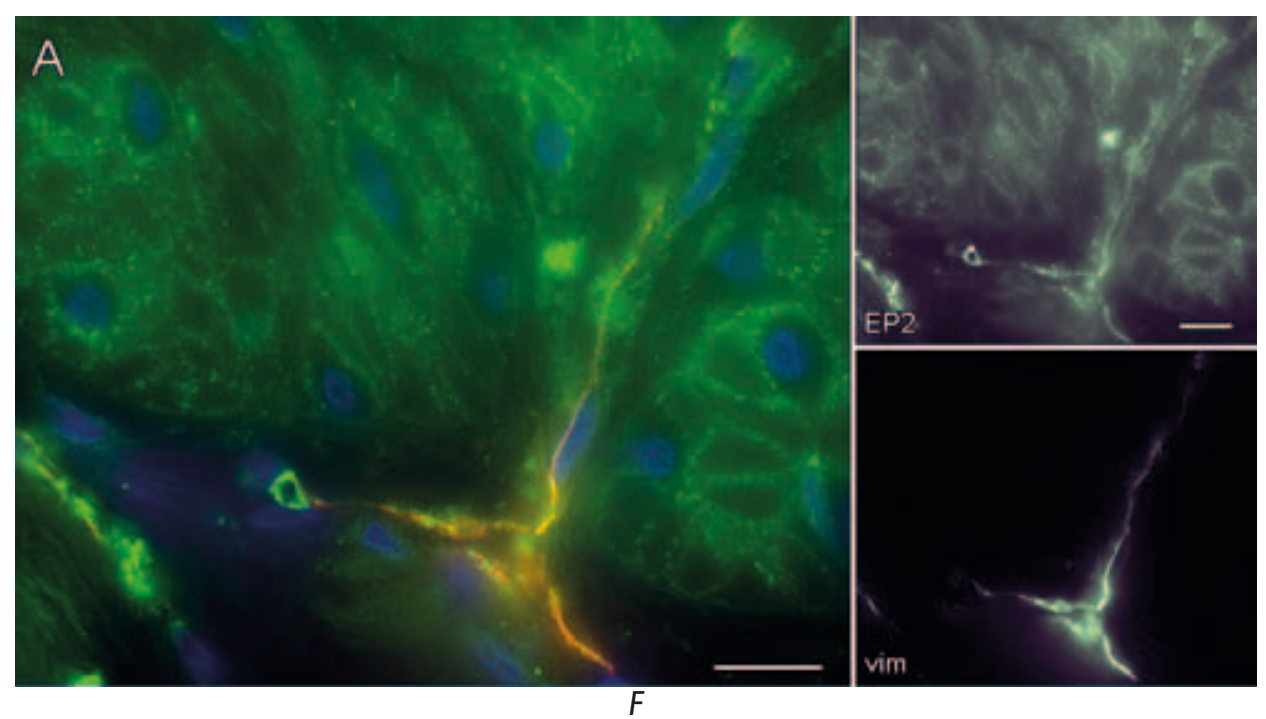

Figure 6. The expression of EP2 in an intramuscular interstitial cell in the lateral wall of the guinea pig bladder. An image taken from a confocal stack of the outer muscle layer of the guinea pig bladder stained with the antibody for EP2 (green) and vimentin (red) is shown. The nuclei of the cells are visualized by a HOECHST nuclei staining (blue). The black and white insets show the individual EP2 and vimentin staining, respectively. Calibration bars: $20 \mu \mathrm{m}$. 
The Distribution of the Prostaglandin E Receptor Type 2 (EP2) in the Detrusor of the Guinea Pig

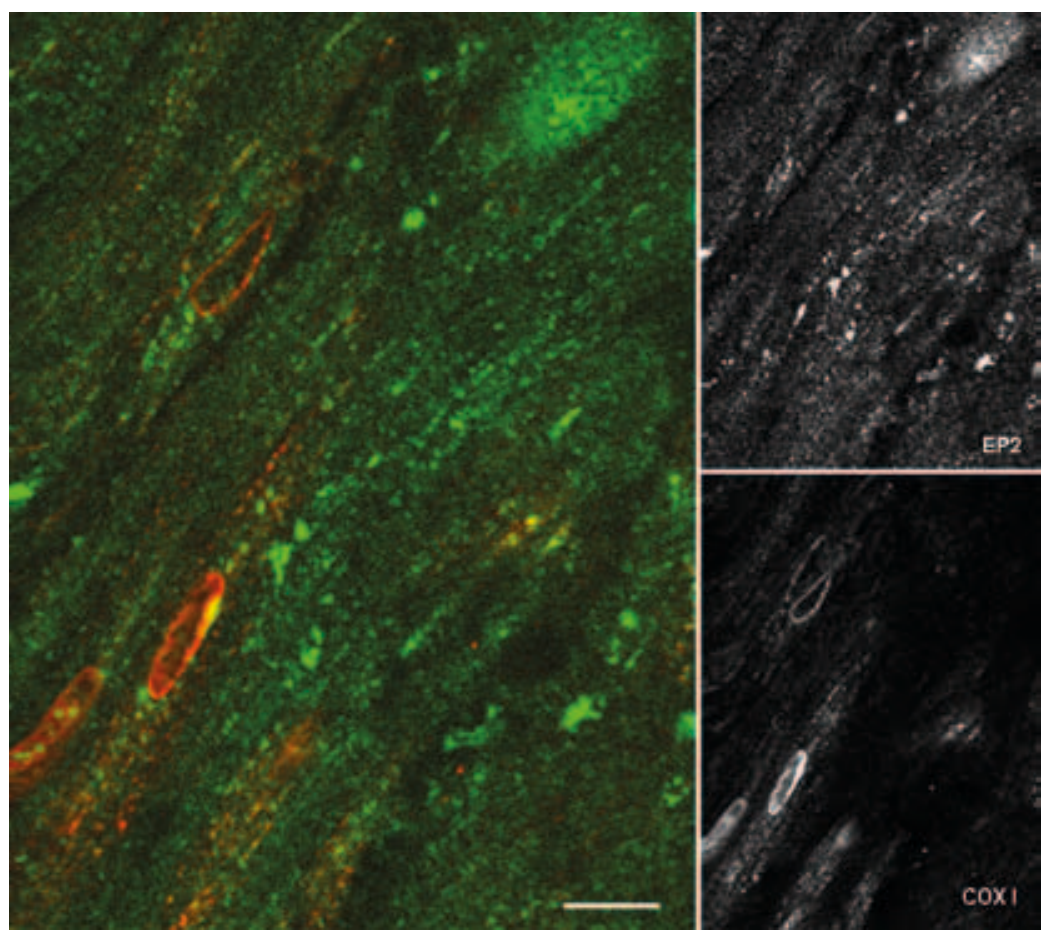

Figure 7. The expression of EP2 and COXI on the muscle bundles of the lateral wall of the guinea pig bladder. An image of the outer muscle layer of the guinea pig bladder stained with the antibody for EP2 (green) and COXI (red) is shown. The black and white insets show the individual EP2 and COXI staining, respectively.

Calibration bars: $20 \mu \mathrm{m}$.

The EP2 staining (green) is especially located on the membrane of muscle cells. The punctuate expression of EP2 on the muscle bundles (green) is further displayed in Figure 7.

It can be noted that some COXI positive interstitial cells (red) are in close proximity of EP2 positive interstitial cells and EP 2 receptors on the near by muscle bundles. Another remarkable observation was that some cells expressing EP2 co-express COXI. This phenomena was seen in about one third of all interstitial cells expressing COXI. A low power image of the guinea pig bladder stained for EP2 (green) and COXI (red) is shown in Figure 8. 


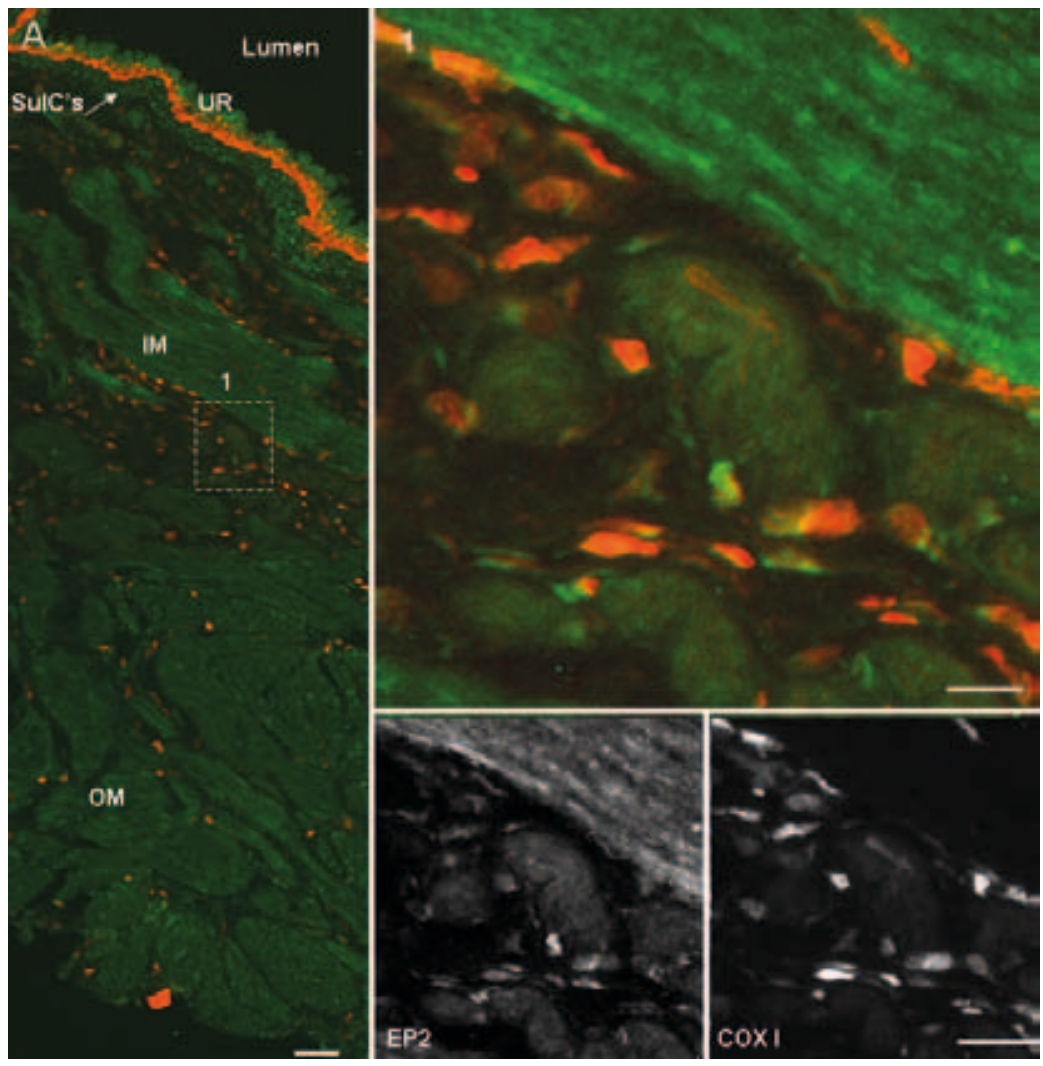

Figure 8. The expression of EP2 and COXI on the muscle bundles of the lateral wall of the guinea pig bladder. A low power image of the guinea pig bladder stained with the antibody for EP2 (green) and COXI (red) is shown in panel A. A region of interest is indicated in panel $A$ and enlarged in the inset $I$. The black and white insets show the individual EP2 and COXI staining, respectively. Calibration bars: $100 \mu \mathrm{m}$ in $\mathrm{A}$ and 20 $\mu \mathrm{m}$ in I.

It can be noted that beside the urothelium, many interstitial cells throughout the muscle layer express COXI. Both COXI positive IM-ICs as well as SM-ICs can be noted.

The findings on EP2 expression within the muscle layers of the guinea pig urinary bladder are summarized in a cartoon shown in Figure 9, which was adapted from our previously published cartoon ${ }^{4}$. In this cartoon it is demonstrated that COXI and the EP2 receptor are expressed on the intramuscular interstitial cells as well as on the muscle bundles. Furthermore the suggested propagation of the PG signal from the urothelium to the muscle layers is visualized. 
The Distribution of the Prostaglandin E Receptor Type 2 (EP2) in the Detrusor of the Guinea Pig

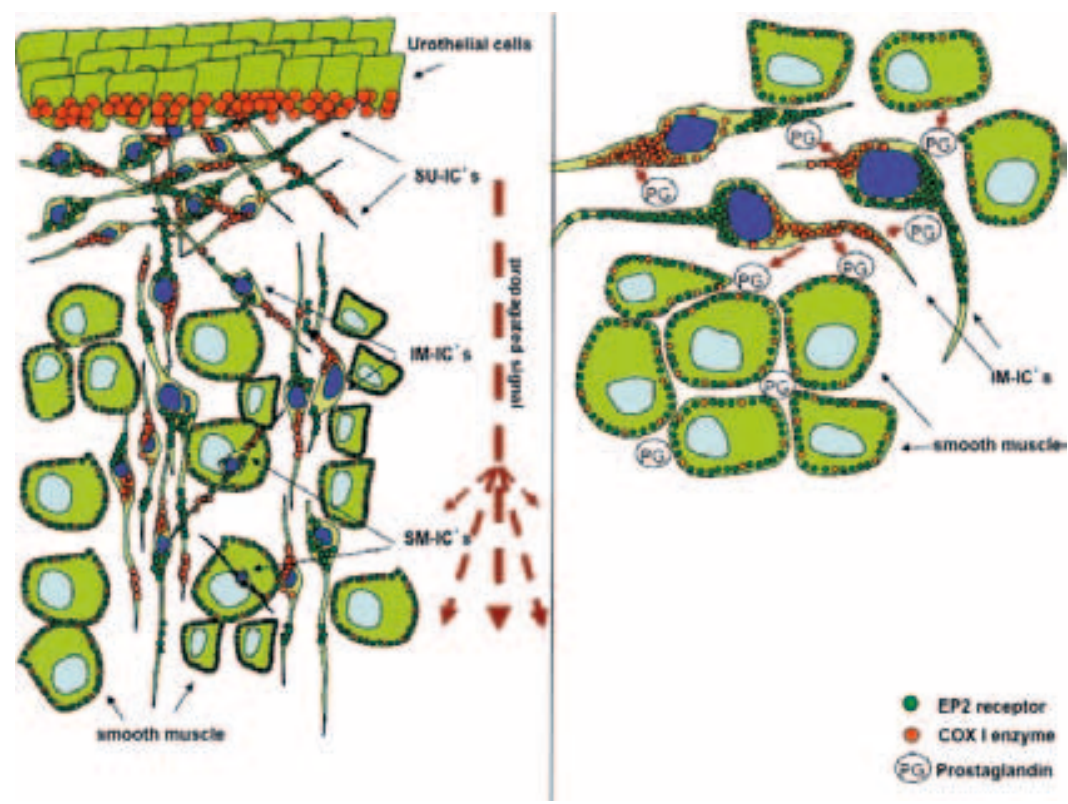

Figure 9. Cartoon of the expression of EP2 and COXI in the muscle bundles. A schematic overview of the lateral wall of the guinea pig bladder and the proposed PG signal transduction. 
Structural and Functional Aspects of Sensory-motor Interaction in the Urinary Bladder

\section{Discussion}

Cyclo-oxygenase inhibiting drugs, have been shown to alter normal voiding function in rats as well as bladder hyperactivity induced by chemical irritation of the lower urinary tract ${ }^{20}$. Furthermore, it is known that intra-arterial administration of $\mathrm{PGE}_{2}$ to the urinary bladder enhances the micturition reflex $^{21}$. Therefore, it has been suggested that PG might act at various sites to regulate or modulate voiding itself and non-voiding activity as well as voiding sensation. Very recently, it was demonstrated that both ATP and $\mathrm{PGE}_{2}$ can induce acetylcholine release from the bladder urothelium '. Therefore, the stretch-induced acetylcholine release, previously described in the bladder ${ }^{22}$, is suggested to be a part of the result of a more complex series of signalling interactions within the urothelium. This complexity is increased because acetylcholine can also modulate $\mathrm{PGE}_{2}$ release. This mechanism is suggested to provide a rapid amplification of urothelial signal output '. In the present study we have focused on EP2 expression in the muscle layers of the guinea pig bladder. EP2 receptor presence on umbrella cells and the sub-urothelial interstitial cells (SU-ICs) has previously been demonstrated ${ }^{4}$. The present study shows that EP2 receptors are further expressed on intramuscular interstitial cells (IM-ICs) and surface muscle interstitial cells (SM-ICs) of the inner- and the outer-muscle layer. Moreover, our stainings show a punctuate low intensity expression of EP2 in guinea pig detrusor muscle bundles. This punctuate appearance deserves more future attention as to whether this might be due to certain areas serving a specific function for example in cell to cell contacts. In the muscle, each of the EP receptors is known to act through different intracellular pathways that involve calcium transport and the stimulation or inhibition of adenylate cyclase to elicit muscle contraction or relaxation depending on the EP subtypes ${ }^{23}$ Figure 7. In general, EPI and EP3 are thought to cause contraction of the smooth muscle, whereas EP2 and EP4 are thought to cause relaxation ${ }^{24,25}$. Each receptor uses a different $\mathrm{G}$-protein coupled transduction system ${ }^{24,25}$. This difference in response of the muscle to each of the EP receptors has been shown for uterine smooth muscle where indeed, activation of EPI and EP3 causes muscle contraction and stimulation of EP2 and EP4 leads to relaxation ${ }^{26}$. Earlier observations showed networks of ICs with short and long processes, running from the urothelium and sub-urothelium through the muscle layers and form connections with other ICs nodes (Figure 8). There is a clear COXI expression in the basal urothelial layer as also reported by earlier papers ${ }^{3,4}$, suggesting the possibility that a PG signal presumably mediated and enhanced by ATP release from the urothelium can be picked up by EPI receptors and be spread further via the IC network ${ }^{3,4}$. Furthermore, these data showed that some COXI positive interstitial cells are in very close proximity of EPI positive interstitial cells and EPI receptors on the nearby muscle bundles. It was described that, PG can be produced in close vicinity, or even within the same cell containing the EPI receptor, which suggested the possibility for a PG signal to be further spread and maybe even augmented ${ }^{3,4}$. Our present data show similar global configurations for the EP2 as was previously shown for the EPI receptor ${ }^{4}$. For EP2, COXI and EP2 immuno-reactivity was in close proximity or even co-expressed in the same cell. Further research is necessary in order to determine the physiological role of this EP2 receptor configuration. However, a spread and 
The Distribution of the Prostaglandin E Receptor Type 2 (EP2) in the Detrusor of the Guinea Pig

augmentation of a signal mediated by PG via EP2 is unlikely as in most smooth muscle preparations, other than the urinary bladder, EP2 has been shown to cause muscle relaxation ${ }^{24}$. In several tissues, EP2 receptors have been shown to couple to a G-protein (Gs), resulting in increased formation of cAMP. In the smooth muscle of different organs, this rise in CAMP through EP2 leads then to muscle relaxation ${ }^{27}$. It is unknown whether this is the predominant effect of EP2 in the detrusor muscle. In several recent reports EP2 action has been shown to be coupled to immune responses or secretion of specific agents, which might also suggest an activation instead of an inhibition ${ }^{28,29}$. The cyclic nucleotide mono-phosphates, cyclic AMP and cyclic GMP, are now generally accepted to be important intracellular regulators of several physiological processes, including smooth muscle function ${ }^{30}$. Rat bladder studies have suggested that beta-adrenoceptor-mediated relaxation is mainly attributed to cAMP-dependent mechanism ${ }^{31,32}$. Moreover, the phosphodiesterase (PDE) type I inhibitor vinpocetine has been shown to have a relaxant effect on human detrusor. This relaxing effect was paralleled by an increase in tissue levels of cyclic AMP and cyclic GMP ${ }^{33}$. There is also some clinical support for the involvement of cAMP in human detrusor control. A randomized double-blind placebo-controlled study, showed that treatment with PDEI inhibitors which lead to a rise in CAMP levels in patients with urgency and urge incontinence was superior to placebo with regard to the clinical outcome parameters micturition frequency, bladder volume at first sensation, bladder volume at voiding desire, maximum detrusor pressure, and voided volume ${ }^{34}$. Moreover, an influence on the above mentioned parameters also suggests a coupling to the bladder sensory system. It can be concluded from these findings that the cyclic AMP-pathway might be of functional significance in the control of detrusor smooth muscle

and indeed have a relaxant effect. The question to be answered now is the possible physiological meaning of the shown EP2 arrangement in relation to the previously shown EPI distribution in the bladder muscle layers. It is known that $\mathrm{PGE}_{2}$, may have multiple and at times apparently opposing functional effects on a given target tissue such avascular smooth muscle ${ }^{35,36}$. Although no functional experiment was included in this study, some possible hypothesis could be put forward. These hypotheses are not based on data and need to be confirmed in future functional studies. One possible explanation could be based on differences in affinity of EP2 and EPI for PG. In fact, it has been shown that the rat EP2 receptor signal transduction pathway is approximately 20 -fold more sensitive for $\mathrm{PGE}_{2}$ than for $\mathrm{EPI}$, as the concentration of $P \mathrm{PE}_{2}$ required to produce a half-maximal response was $37 \mathrm{nM}$ for EP2 against $2.1 \mathrm{nM}$ for EPI ${ }^{37}$. Furthermore, in order to determine the affinity of $P G E_{2}$ for $E P I$ and $E P 2$, saturation analysis has been done showing KD values of $P G E_{2}$ for EPI and EP2 be around 24 and $5 \mathrm{nM}$, respectively ${ }^{37}$. Therefore, it can be hypothesized that under normal conditions, $\mathrm{PGE}_{2}$ levels in the detrusor might be such that the inhibiting EP2 effect predominates the activating EPI effect, which might result in a stabilization of the muscle layer. Under certain circumstances, the $\mathrm{PGE}_{2}$ levels may increase above a certain threshold level in which case the contractile EPI effect becomes more predominant. Small sub-threshold increases of PGE ${ }_{2}$ might, in such a case, be due to subtitle bladder wall irritation, inflammation or stretch. The exact 
Structural and Functional Aspects of Sensory-motor Interaction in the Urinary Bladder

role of the EP2 receptor in the bladder wall remains to be determined by future analysis of the activation, signal propagation and bladder sensory pathways by functional studies. The $\mathrm{PGE}_{2}$ signal is suggested to be spread out through suburothelial and intramuscular interstitial cells expressing EPI. The narrow balance between the contractile EPI and the possible relaxant EP2 effect might be a system that modulates the autonomous, non-voiding contractions and relaxation which can become disrupted when $\mathrm{PGE}_{2}$ levels reach certain higher levels. This idea is supported by earlier studies showing that frequency and amplitude of the non-voiding activity is reduced by stimulation of an adrenergic/cAMP-linked mechanism ${ }^{38}$.

\section{Conclusion}

This morphological study indicates that the EP2 receptor is expressed on muscle interstitial cells and on muscle bundles. The proximity of the COXI positive interstitial cells to EP2 positive cells suggests that PG targeting these EP2 receptors can be produced locally. It is hypothesized that the $\mathrm{PGE}_{2}$ concentration in the tissue might be responsible for the threshold level setting of interstitial cell excitability. However, functional experiments are needed in order to confirm this hypothesis and the physiological significance of the EPI and EP2 receptors in the bladder. 
The Distribution of the Prostaglandin E Receptor Type 2 (EP2) in the Detrusor of the Guinea Pig

\section{References}

I. Nile, C.J. \& Gillespie, J.I. Interactions between cholinergic and prostaglandin signaling elements in the urothelium: role for muscarinic type 2 receptors. Urology 79, 240 e217-223 (2012).

2. Kobayter, S., Young, J. \& Brain, K. Prostaglandin E(2) induces spontaneous rhythmic activity in mouse urinary bladder independently of efferent nerves. BrJ Pharmacol I65, 40I-4I3 (2012).

3. Rahnama'i, M.S., et al. The relationship between prostaglandin E receptor I and cyclooxygenase I expression in guinea pig bladder interstitial cells: proposition of a signal propagation system. J Urol 185, 315-322 (2011).

4. Rahnama'i, M.S., et al. Prostaglandin receptor EPI and EP2 site in guinea pig bladder urothelium and lamina propria. J Urol 183, I24I-1247 (2010).

5. Maggi, C.A., et al. Evidence for the involvement of arachidonic acid metabolites in spontaneous and drug-induced contractions of rat urinary bladder.J Pharmacol Exp Ther 230, 500-5I3 (1984).

6. de Jongh, R., et al. The localization of cyclo-oxygenase immuno-reactivity (COXI-IR) to the urothelium and to interstitial cells in the bladder wall. J Cell Mol Med I3, 3069-308I (2009).

7. Mikhailidis, D.P., Jeremy, J.Y. \& Dandona, P. Urinary bladder prostanoids--their synthesis, function and possible role in the pathogenesis and treatment of disease. The Journal of urology 137, 577-582 (1987).

8. Narumiya, S., Sugimoto, Y. \& Ushikubi, F. Prostanoid receptors: structures, properties, and functions. Physiological reviews 79, I193-1226 (1999).

9. McCafferty, G.P., Misajet, B.A., Laping, N.J., Edwards, R.M. \& Thorneloe, K.S. Enhanced bladder capacity and reduced prostaglandin E2-mediated bladder hyperactivity in EP3 receptor knockout mice. Am J Physiol Renal Physiol 295, F507-5I4 (2008).

10. Andersson, K.E. \& Forman, A. Effects of prostaglandins on the smooth muscle of the urinary tract. Acta Pharmacol Toxicol (Copenh) 43 Suppl 2, 90-95 (1978).

II. Birder, L.A. Urinary bladder urothelium: molecular sensors of chemical/thermal/mechanical stimuli. Vascul Pharmacol 45, 221-226 (2006).

12. McCloskey, K.D. Interstitial cells in the urinary bladder--localization and function. Neurourol Urodyn 29, 82-87 (2010).

13. de Jongh, R., et al. Alterations to network of NO/cGMP-responsive interstitial cells induced by outlet obstruction in guinea-pig bladder. Cell and tissue research 330, 147-160 (2007).

14. Gillespie, J.I., Markerink-van Ittersum, M. \& De Vente, J. Endogenous nitric oxide/cGMP signalling in the guinea pig bladder: evidence for distinct populations of sub-urothelial interstitial cells. Cell and tissue research 325, 325-332 (2006).

I5. Gillespie, J.I., Markerink-van Ittersum, M. \& De Vente, J. Interstitial cells and cholinergic signalling in the outer muscle layers of the guinea-pig bladder. BJU international 97, 379-385 (2006).

16. de Jongh, R., et al. The effects of exogenous prostaglandins and the identification of constitutive cyclooxygenase I and II immunoreactivity in the normal guinea pig bladder. BJU Int I00, 4I9-429 (2007).

17. Gillespie,J.I.,Harvey, I.J.\& Drake,M.J.Agonist- and nerve-induced phasic activity in the isolated whole bladder of the guinea pig: evidence for two types of bladder activity. Experimental physiology 88, 343-357 (2003). 
Structural and Functional Aspects of Sensory-motor Interaction in the Urinary Bladder

18. Grol, S., Essers, P.B., van Koeveringe, G.A., de Vente, J. \& Gillespie, J.I. Ubiquitin hydrolase (protein gene product 9.5 ) in the obstructed bladder: evidence for tissue remodelling involving a subset of interstitial cells. BJU international 104, 698-706 (2009).

19. Palea, S., et al. Pharmacological characterization of thromboxane and prostanoid receptors in human isolated urinary bladder. BrJ Pharmacol I24, 865-872 (1998).

20. de Groat, W.C. \& Yoshimura, N. Pharmacology of the lower urinary tract. Annual review of pharmacology and toxicology 4I, 69I-72I (200I).

21. Ishizuka, O., Mattiasson, A. \& Andersson, K.E. Prostaglandin E2-induced bladder hyperactivity in normal, conscious rats: involvement of tachykinins? J Urol I53, 2034-2038 (1995).

22. Yoshida, M., et al. Non-neuronal cholinergic system in human bladder urothelium. Urology 67, 425-430 (2006).

23. Ponglowhapan, S., Church, D.B. \& Khalid, M. Expression of prostaglandin E receptor subtypes in the canine lower urinary tract varies according to the gonadal status and gender. Theriogenology 74,1450 1466 (2010).

24. Coleman, R.A., Smith, W.L. \& Narumiya, S. International Union of Pharmacology classification of prostanoid receptors: properties, distribution, and structure of the receptors and their subtypes. Pharmacol Rev 46, 205-229 (1994).

25. Ikeda, M., Kawatani, M., Maruyama, T. \& Ishihama, H. Prostaglandin facilitates afferent nerve activity via EPI receptors during urinary bladder inflammation in rats. Biomed Res 27, 49-54 (2006).

26. Astle, S., Thornton, S. \& Slater, D.M. Identification and localization of prostaglandin E2 receptors in upper and lower segment human myometrium during pregnancy. Mol Hum Reprod II, 279-287 (2005).

27. Breyer, R.M., Bagdassarian, C.K., Myers, S.A. \& Breyer, M.D. Prostanoid receptors: subtypes and signaling. Annu Rev Pharmacol Toxicol 4I, 66I-690 (200I).

28. Fedyk, E.R. \& Phipps, R.P. Prostaglandin E2 receptors of the EP2 and EP4 subtypes regulate activation and differentiation of mouse B lymphocytes to IgE-secreting cells. Proc Natl Acad Sci U S A 93, 1097810983 (1996).

29. Harizi, H., Limem, I. \& Gualde, N. CD40 engagement on dendritic cells induces cyclooxygenase-2 and EP2 receptor via p38 and ERK MAPKs. Immunol Cell Biol 89, 275-282.

30. Antoni, F.A. Molecular diversity of cyclic AMP signalling. Front Neuroendocrinol 2I, I03-132 (2000).

3I. Uchida, H., Shishido, K., Nomiya, M. \& Yamaguchi, O. Involvement of cyclic AMP-dependent and -independent mechanisms in the relaxation of rat detrusor muscle via beta-adrenoceptors. Eur J Pharmacol 518, 195-202 (2005).

32. Yamaguchi, O. \& Chapple, C.R. Beta3-adrenoceptors in urinary bladder. Neurourol Urodyn 26, 752-756 (2007).

33. Truss, M.C., Uckert, S., Stief, C.G., Kuczyk, M. \& Jonas, U. Cyclic nucleotide phosphodiesterase (PDE) isoenzymes in the human detrusor smooth muscle. I. Identification and characterization. Urol Res 24 , 123-128 (1996).

34. Truss, M.C., et al. Phosphodiesterase I inhibition in the treatment of lower urinary tract dysfunction: from bench to bedside. World J Urol 19, 344-350 (200I). 
The Distribution of the Prostaglandin E Receptor Type 2 (EP2) in the Detrusor of the Guinea Pig

35. Bonvalet, J.P., Pradelles, P. \& Farman, N. Segmental synthesis and actions of prostaglandins along the nephron. Am J Physiol 253, F377-387 (1987).

36. Smith, W.L. Prostanoid biosynthesis and mechanisms of action. Am J Physiol 263, FI8I-191 (1992).

37. Boie, Y., et al. Molecular cloning and characterization of the four rat prostaglandin E2 prostanoid receptor subtypes. Eur J Pharmacol 340, 227-24I (1997).

38. Gillespie, J.I. Noradernaline inhibits autonoumous activity in the isolated guinea pig bladder BJU international feb, 40I-409 (2004). 

Chapter 3

\section{Distribution and Sub-types of Afferent Fibre in the Mouse Urinary Bladder}

Bart T. Biallosterski, Mohammad S. Rahnama'i, Philip E.V. Van Kerrebroeck,

Gommert A. van Koeveringe, James I. Gillespie and Stefan G.G. de Wachter

Submitted Journal of Chemical Neuranatomy 


\section{Abstract}

Aim

Increased afferent fibre activity contributes to pathological conditions such as the overactive bladder syndrome. Historically nerve fibres running near the urothelium are considered to be afferent as no efferent system has yet been described. The aim of this study was to identify subtypes of afferent nerve fibres in the mouse bladder wall based on morphological criteria and analyse regional differences.

\section{Materials and Methods}

27 bladders of six months old C57BL6 mice were removed and tissues were processed for immunohistochemistry. Cryostat sections were cut and stained for Protein Gene Product 9.5 (PGP), anticalcitonin gene related polypeptide (CGRP), anti-Neurofilament (NF), anti-vesicular acetylcholine transporter (VAChT) and anti-neuronal nitric oxide synthase (nNOS).

\section{Results}

In the lamina propria different types of afferent nerve fibre were found, i.e. immunoreactive (IR) to; CGRP, NF, VAChT, and/or nNOS. At the bladder base the lamina propria was more densely innervated by CGRP-IR and VAChT-IR nerve fibres, then at the lateral wall. NF- and nNOS nerves were sparsely distributed in the lamina propria throughout the bladder. At the lateral wall the inner muscle is densely innervated by CGRP-IR nerve fibres. NF, VAChT and nNOS nerves were evenly distributed in the different muscle layers throughout the bladder. Nerve fibre terminals expressing CGRP and NF were found within the extra-mural ganglia at the bladder base.

\section{Conclusions}

Different types of afferent nerve fibres are identified in the lamina propria of the mouse bladder. At the bladder base the lamina propria is more densely innervated than the lateral wall by CGRP-IR and VACHT-IR afferent nerve fibres. CGRP and NF afferent nerve fibres in the muscle layer probably relay afferent input to external ganglia located near the bladder base. The identification of different afferent nerves in the lamina propria suggests a functional heterogeneity of the afferent nerve fibres in the urinary bladder. 


\section{Introduction}

There is accumulating evidence that aberrant afferent activity plays an important role in the overactive bladder syndrome $(O A B)$ '. During bladder filling, sensory information is relayed to the central nervous system by the pudendal, pelvic and hypogastric nerves. Each of these nerves contains different types of afferent fibres, which can be identified and differentiated functionally and structurally. They consist of small myelinated A $\delta$ fibres, mainly located in detrusor muscle, and unmyelinated $C$ nerve fibres innervating all layers of the bladder wall. These types can functionally be distinguished based on the conduction velocity, or their response to mechanosensitive or chemosensitive stimuli. However, no functional relationship between conduction velocity and receptor type has been described ${ }^{2}$. Histological studies have shown that bladder afferent nerve fibres originate from the dorsal root ganglia (DRG) and contain multiple neuropeptides, e.g. Substance P (SP), Neurofilament protein (NF) and neuronal Nitric Oxide synthase (nNOS) and Calcitonin Gene Related Peptide (CGRP) '. The exact distribution of terminal endings of these peptide-containing afferents is still largely unknown but they appear to be more densely present in the lamina propria just beneath the urothelium ${ }^{3}$. Nerve fibres in the lamina propria near the urothelium are considered to be afferent fibres as no efferent system has yet been identified ${ }^{3}$. Furthermore, the afferent innervation of the bladder neck and trigone is higher in density than the lateral wall and dome ${ }^{4}$. Regional variation in the suburothelial cholinergic innervation has been observed in the guinea pig urinary bladder, suggesting functional differentiation between the lateral wall and bladder base ${ }^{5}$. Moreover, co-localization of different neuropeptides has been shown also suggesting a functional heterogeneity ${ }^{6}$. This may explain why some fibres respond to a wide range of mechanical and chemical stimuli, such as stretch, pain, chemical modulation and motor/sensory elements ${ }^{7}$. In the mouse bladder, using electrophysiological techniques, at least four functionally different types of afferent nerve fibre have been identified ${ }^{8}$. Based on their responses to mechanical stimuli (perpendicular von Frey probing, urethral stroking, stretch), urothelial, muscular/urothelial, muscular, and serosal afferents were found. Structurally Lagou et al. already described the presence of CGRP and $\mathrm{nNOS}$ nerve fibres in the lamina propria but no specific attention was paid to regional differences ${ }^{9}$. In this study, we attempt to identify afferent nerves in the mouse bladder and describe differences in the bladder base and lateral wall with particular attention to the lamina propria and urothelium.

\section{Materials and Methods}

All experimental procedures were approved by the local animal ethical committee of the Maastricht University, and were conducted in accordance with Dutch governmental guidelines. In total 27 six months old C57BL/6 male mice were used. The animals were housed in a temperature and light (I2 $\mathrm{h}$ light/dark cycle) controlled room and allowed free access to food and water. Mice were killed by cervical dislocation. The urinary bladder was removed and divided into two pieces, dome/lateral wall 
Structural and Functional Aspects of Sensory-motor Interaction in the Urinary Bladder

and base and fixed in an ice-cold solution of $4 \%$ freshly prepared depolymerised paraformaldehyde in 0.1 M phosphate buffer ( $\mathrm{pH} 7.4)$ for 2 hours at $4^{\circ} \mathrm{C}$. Afterwards the tissue was washed at $4-8{ }^{\circ} \mathrm{C}$ in 0.1 M phosphate buffer containing $10 \%$ sucrose $(24 \mathrm{~h}), 20 \%$ sucrose $(24 \mathrm{~h})$ and $30 \%$ sucrose $(24 \mathrm{~h})$. Tissue was snap frozen with isopentane cooled in liquid nitrogen and placed in Tissue-Tek O.C.T. compound (Bayer Corporation, Pittsburgh, PA, USA) to form a single block. Cryostat sections (I0 $\mu \mathrm{m})$ were cut in a manner so that each section was perpendicular to the urothelial surface. Sections were then thawed onto chrome-alum-gelatine-coated slides and processed for immunohistochemistry.

\section{Immunohistochemistry}

Sections were dried for $30 \mathrm{~min}$ at room temperature followed by three washes with Tris-buffered saline (TBS; $\mathrm{pH}$ 7.6) for $5 \mathrm{~min}$. and thereafter incubated overnight with primary antibodies at $4{ }^{\circ} \mathrm{C}$. Primary antibodies were diluted in TBS containing $0.3 \%(\mathrm{v} / \mathrm{v})$ Triton X-100 (TBS-T). We used several different antibodies to stain for afferent nerves and counterstained them with the general nerve marker Protein Gene Product 9.5 (PGP). Chicken anti-PGP 9.5 primary antibody was used to visualize PGP (dilution I:3000, Abcam, Cambridge, UK). Rabbit anti-calcitonin gene related polypeptide (CGRP, dilution I:I000, Calbiochem, Merck Biosciences, Nottingham, UK) and rabbit anti-Neurofilament (NF, dilution I:400, Millipore, Billerica, MA, USA) were used to visualize afferent nerves. The rabbit antibody against the vesicular acetylcholine transporter (VAChT, dilution I:I000, Sigma-Aldrich) was used to visualize cholinergic fibres, neuronal nitric oxide synthase was visualized using sheep anti-nNOS antibody ( $\mathrm{nNOS}$, dilution I:I000, generous gift from Dr. P.C. Emson, Babraham Institute, Cambridge, UK). After overnight incubation with the primary antibodies, sections were washed in TBS-T, TBS and TBS-T; each wash step lasted $15 \mathrm{~min}$. Alexa Fluor 488 donkey anti-chicken $\lg \mathrm{G}(\mathrm{H}+\mathrm{L})$ conjugate (Molecular Probes) was used to visualize PGP 9.5 diluted I: 100 in TBS-T. VAChT, NF and CGRP rabbit primary antibodies were visualized with Alexa Fluor 594 donkey anti-rabbit IgG conjugate (Molecular Probes), diluted I:I00 in TBS-T. Sheep nNOS primary antibody was visualized with Alexa Fluor 594 donkey antisheep $\lg G(\mathrm{H}+\mathrm{L})$ conjugate (Molecular Probes). Sections were incubated with the secondary antibodies for $90 \mathrm{~min}$ at RT in the dark. After washing, the sections were mounted with TBSglycerol (80\%). Staining was done in duplicate and repeated at least on 2 separate days. Sections were photographed using an Olympus $A \times 70$ microscope, using $\times 10, \times 20$ and $\times 40$ (oil) objectives. For the detection of Alexa 488 fluorescence we used a narrow band-pass MNIBA-filter and for the detection of Alexa 594 we used a filter with a narrow excitation band, the U-M4I007A filter (both filters are from Chroma Technologies). The microscope was equipped with a cooled CCD Olympus Digital video camera F-view. Images were acquired as 16 bits images using the computer program cell-P® (Olympus, Germany). The number of grey values was reduced to 4095 using a linear function. Images were arranged with the program Microsoft PowerPoint 2003 (Microsoft Corporation, Seattle, WA, USA). 


\section{Results}

PGP-IR nerves were uniformly present in the muscle layers of both bladder base and lateral wall. At the lateral wall the lamina propria was sparsely innervated with PGP-IR nerve fibres compared to the bladder base.

At the bladder base the lamina propria was densely innervated by CGRP-immunoreactive (IR) fibres (Figure I, panel A, SU). Whereas at the lateral wall the amount of CGRP-IR nerve fibres in the lamina propria was reduced (Figure I, panel B). The muscle was sparsely innervated by CGRP-IR nerve fibres at the bladder base, interestingly the inner muscle layer showed more CGRP-IR fibres than the outer muscle layer (Figure I, panel A). At the lateral wall the inner muscle was also densely innervated by CGRP-IR nerve fibres (Figure I).
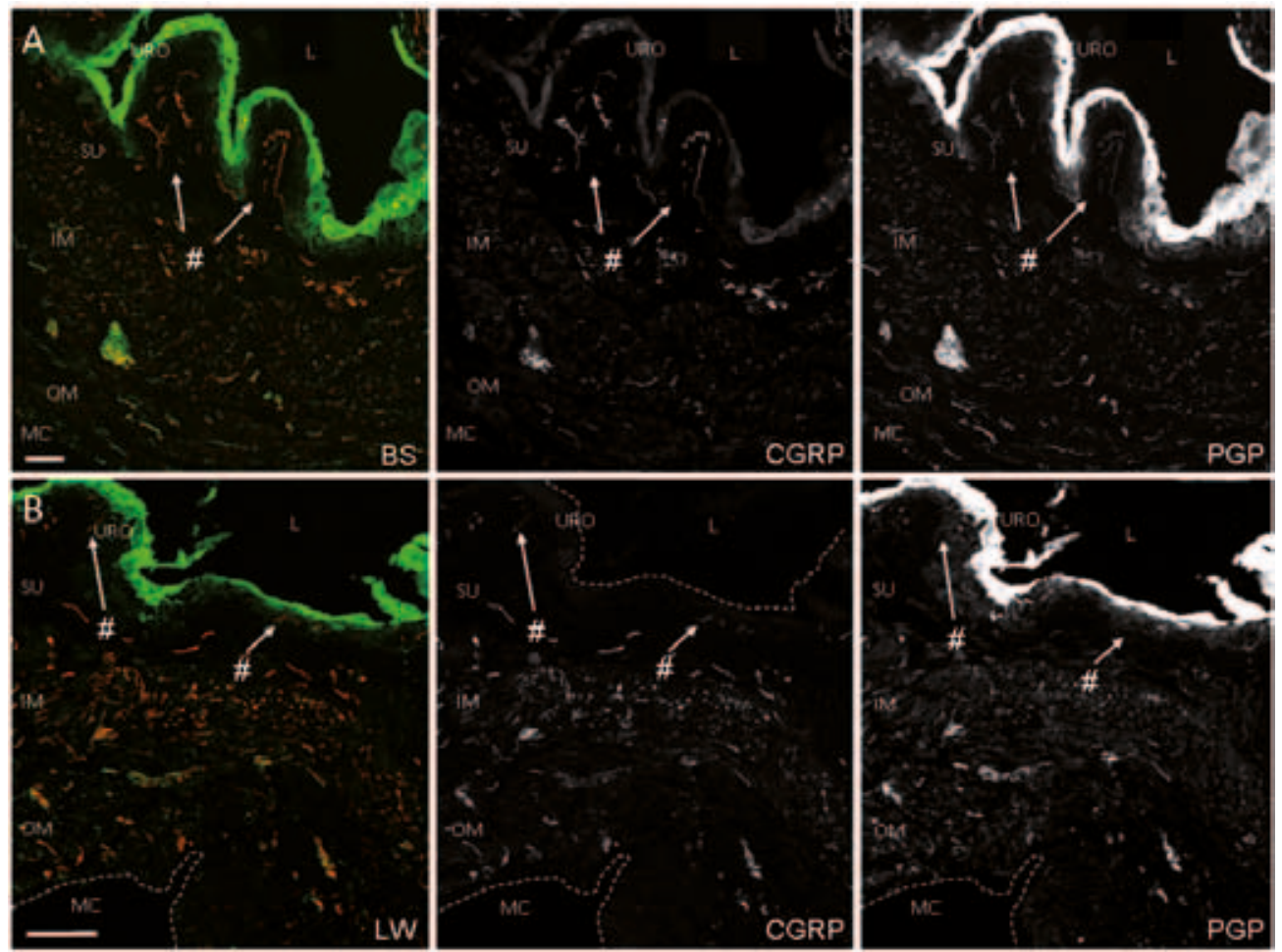

Figure I. Photomicrographs taken from sections of the base (A) and lateral wall (B) of a C57BL/6 mouse bladder.The sections are double stained for PGP (green) and CGRP (red). In both panels A and B the double staining is shown with the black and white image of CGRP and PGP next to it.The nerves in the suburothelium (\# arrows) are more densely innervated in the base (A) compared to the lateral wall (B). In the lateral wall (B) a clear difference between CGRP nerve distribution in the inner and outer muscle layer can be seen. More CGRP is visible in the inner muscle layer compared to the outer. $\mathrm{URO}=$ urothelium; $\mathrm{L}=\mathrm{Lumen} ; \mathrm{SU}=$ suburothelium; $\mathbb{I M}=$ inner muscle layer; $\mathrm{OM}=$ outer muscle layer; $M C=$ muscle coat. Calibration bars: $25 \mu \mathrm{m}$. 

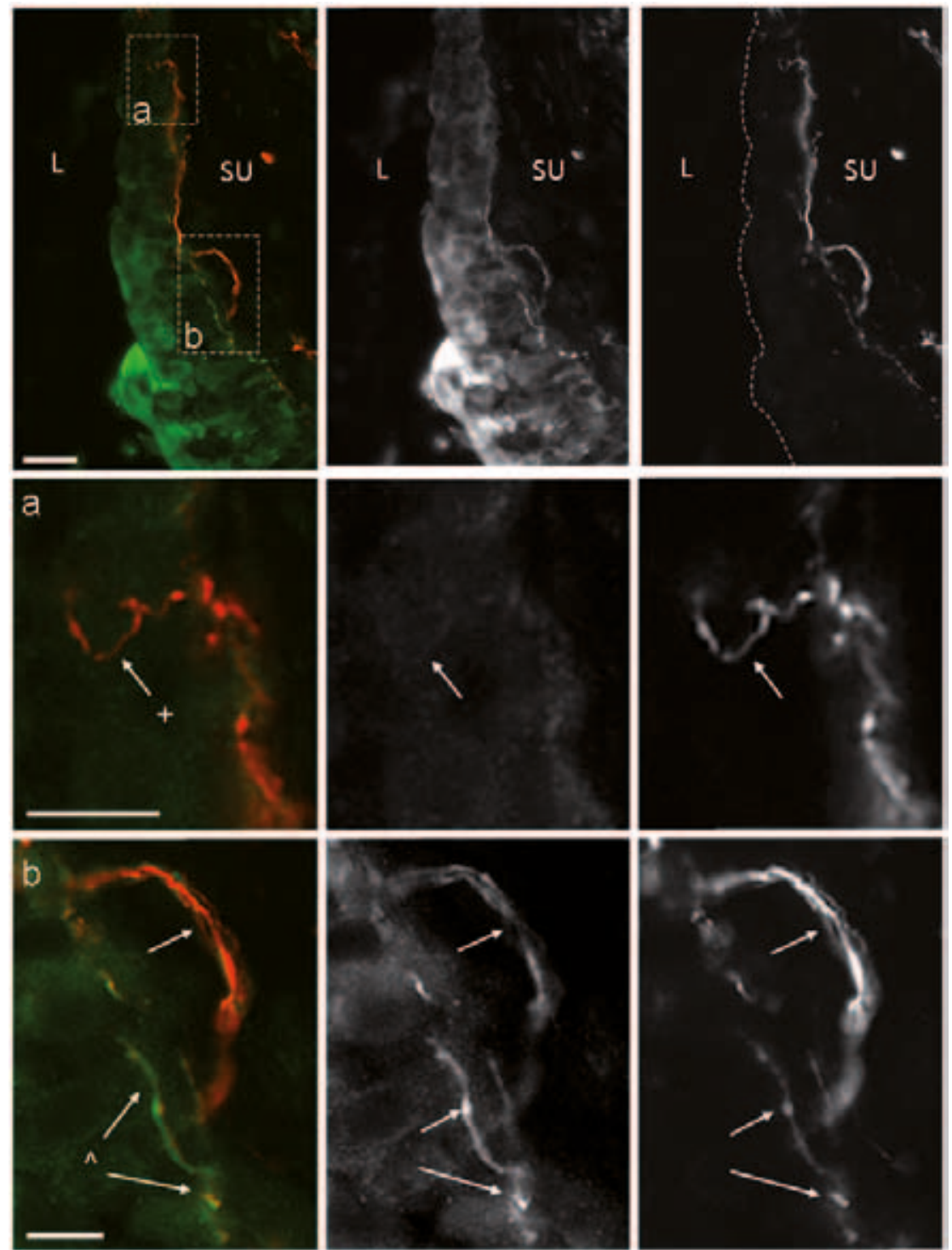

Figure 2. Photomicrograph showing a magnified region of the suburothelium in the bladder base. The sections are double stained for PGP (green) and CGRP (red). Panels B and C show magnifications of the squares in A. In panel B the nerve fibre $(+$ arrow) primarily stains for CGRP and this fibre runs all the way into the urothelial layer. Panel $C$ shows fibres staining for CGRP and PGP marked with \# and ${ }^{\wedge}$. These fibres do not seem to co-localise, but are seen to run around each other.The bottom CGRP fibre has several varicosities possibly indicating nerve terminals ( ${ }^{\wedge}$ arrows). $\mathrm{L}=$ Lumen and SU = suburothelium. Calibration bar: $25 \mu \mathrm{m}$ in $\mathrm{A}, 10 \mu \mathrm{m}$ in $\mathrm{C}$ and $\mathrm{D}$. 
In the lamina propria in both the bladder base and the lateral wall, CGRP-IR nerve fibres with varicosities ran immediately below the basal layer of the urothelium, at some spots possibly penetrating this layer right underneath the umbrella cells (Figure 2, panel A and panel (a) and (b)). Higher magnification revealed that not all of these fibres were PGP-IR positive. The selectivity of the first PGP antibody was cross checked by a second primary PGP Ab which revealed the same results (anti-rabbit PGP, I:3000, Sigma-Aldrich) (Figure 2, panel (a)). PGP-IR and CGRP-IR nerve fibres were seen running in close proximity to each other in the sub-urothelial layer (Figure 2, panel (b)).

Neurofilament (NF) was used to stain Ad afferent nerve fibres ${ }^{10}$. These fibres were few in number and primarily located in the muscle layers. A small amount of NF-IR nerve fibres were seen running in the lamina propria, not close to the urothelium (Figure 3, panel A and B). The muscle layers were innervated by both small and large NF-IR nerve fibres. No differences were observed between the bladder base and lateral wall. Panel $C$ shows a NF-IR nerve fibre in the muscle layers in close proximity to PGP-IR nerve fibres, although these fibres did not co-localize.
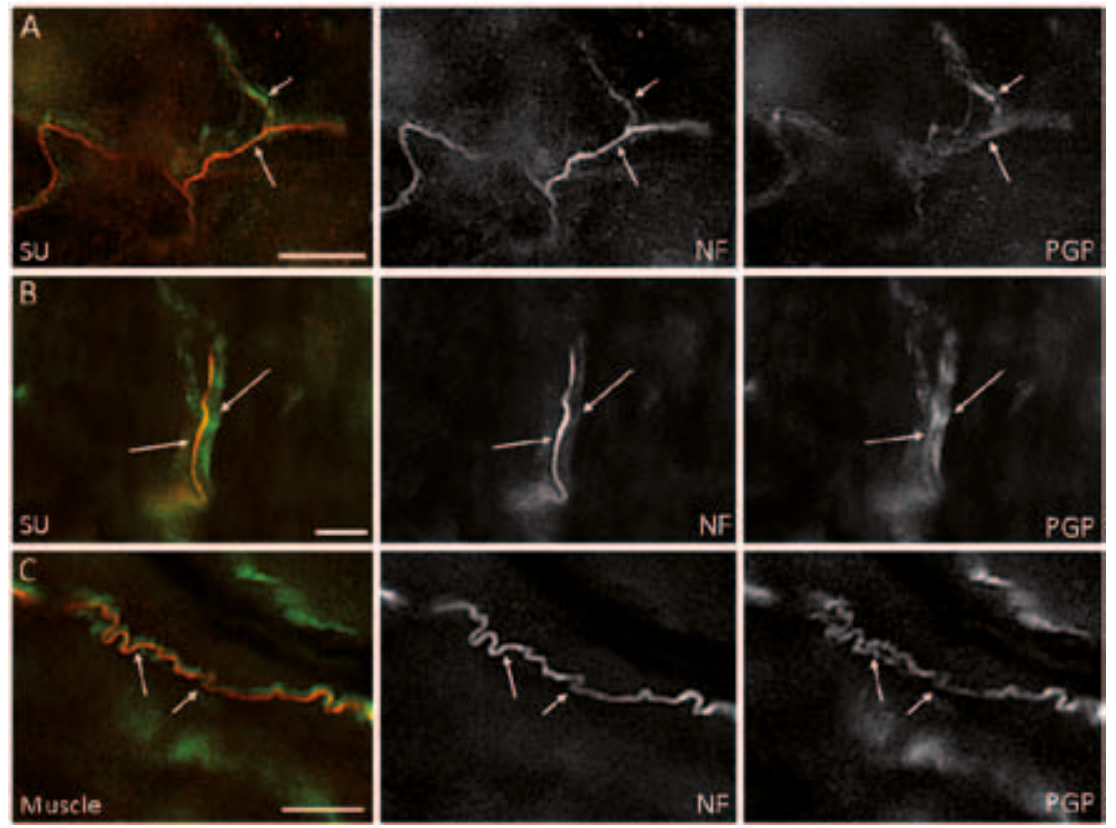

Figure 3. Photomicrographs showing a section of the bladder.The section is double stained for PGP (green) and NF (red). Nerve fibres which stain for NF are found in the suburothelium (\# arrows) and in the muscle layer (arrows). Calibration bar:50 $\mu \mathrm{m}$. 

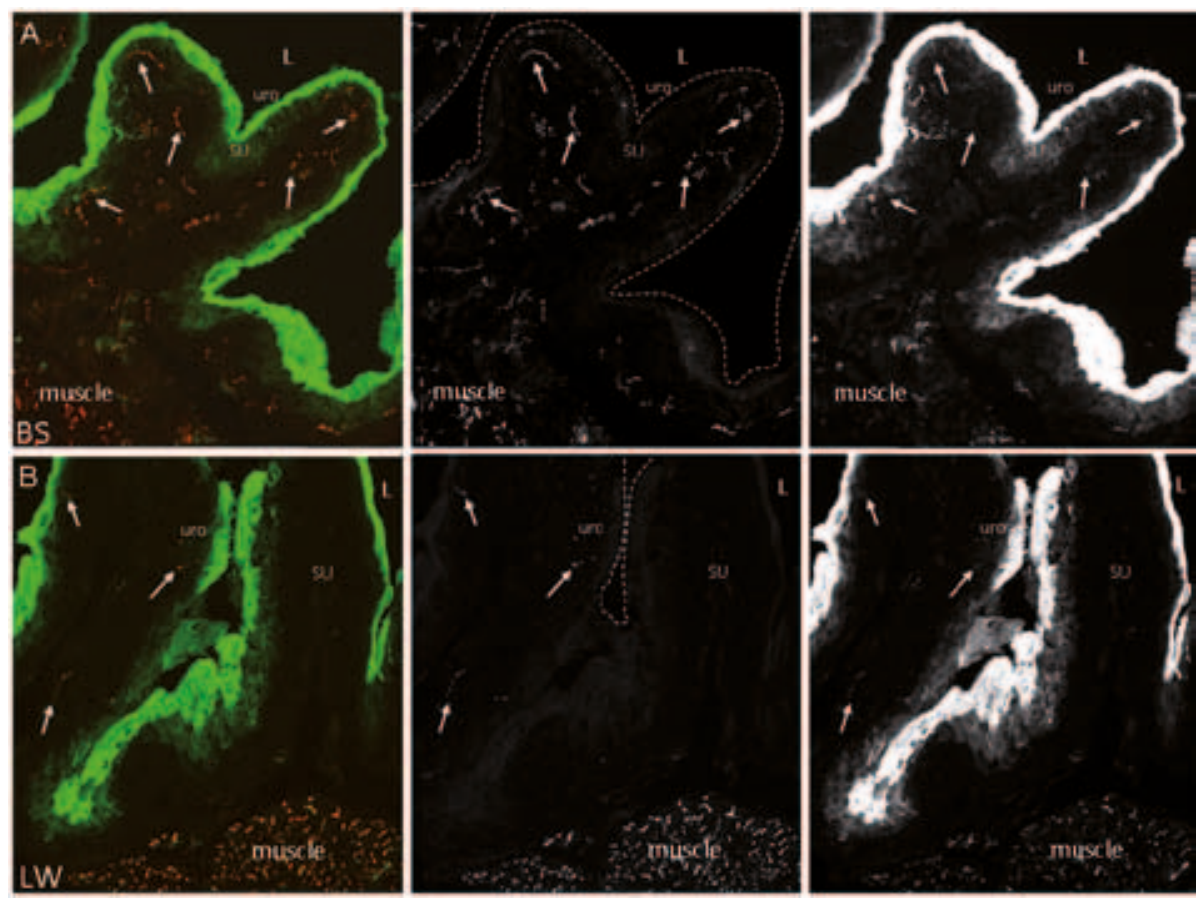

Figure 4. Photomicrographs taken from sections of the base $(A)$ and lateral wall $(B)$ of the bladder.The sections are double stained for PGP (green) andVAChT (red). The nerves in the suburothelium (arrows) are more densely innervated in the base (A) compared to suburothelial nerves (arrows) in the lateral wall (B). Nerve distribution in the muscle is uniform throughout both bladder base and lateral wall. Calibration bar: $50 \mu \mathrm{m}$.

VAChT-IR nerve fibres densely innervate the sub-urothelium of the bladder base, here these fibres are presumed to be afferent (Figure 4, panel A, arrows). Similar to the CGRP-IR nerve fibres (Figure I), the amount of VAChT-IR nerve fibres in the lamina propria at the lateral wall was reduced, compared to the bladder base (Figure 4, panels $A$ and $B$ ).

In the muscle layers intense VAChT-IR nerve fibres innervate both the outer- and inner muscle layers. In the sub-urothelium VAChT-IR nerve fibres, with characteristic varicosities, were visible (Figure 5). As with CGRP-IR and NF-IR nerve fibres, PGP-IR nerve fibres ran in close proximity to the VAChT-IR nerve fibre (Figure 5, panel B). 

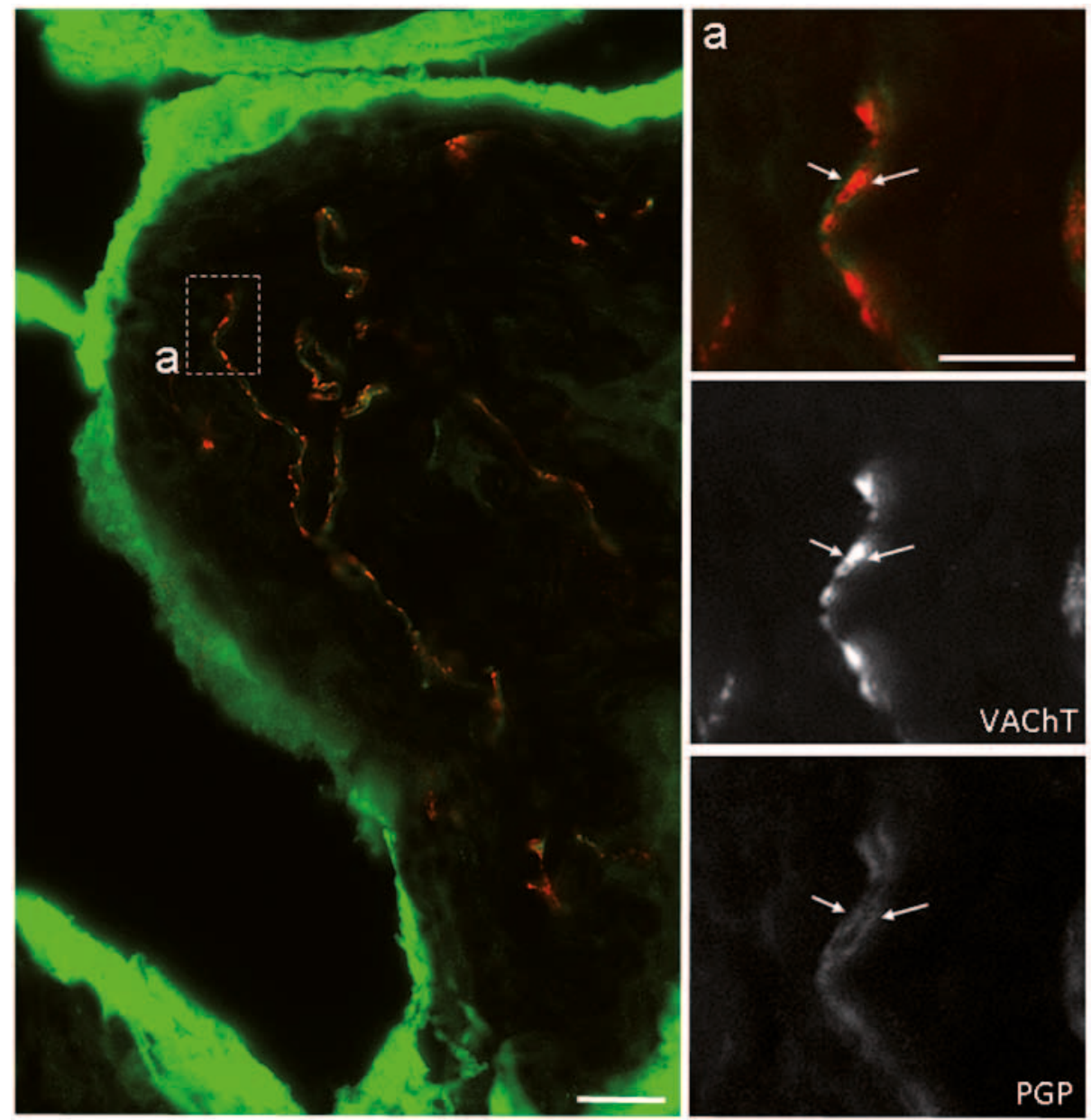

Figure 5. Photomicrographs of a bladder section. The section is double stained for PGP (green) andVAChT (red). Panel A shows nerve fibres in the suburothelium. The square is magnified in panel B.The nerve fibre stains for both PGP andVAChT. In VAChT some varicosities are visible (\# arrow). These varicosities do not show up in PGP. Panel C is a magnification of a nerve fibres end from panelA.The end stains inVAChT and PGP (+arrows) and might be a nerve terminal. Calibration bars: $20 \mu \mathrm{m}$.

nNOS-IR nerve fibres were present in all layers of the bladder wall. Most of these nerve fibres were found in the muscle layers. Like VAChT-IR nerve fibres, nNOS-IR nerve fibres in the lamina propria were also presumed to be afferent (Figure 6). Only a few fibres were found in the lamina propria with none of these fibres co-localizing with PGP-IR nerve fibres. nNOS-IR nerve fibres ran closer to the urothelium than the NF-IR nerve fibres (Figure 6, panel B and D), but not as close as the VAChT-IR or the CGRP-IR nerve fibres (Figure 5 and Figure 2). 

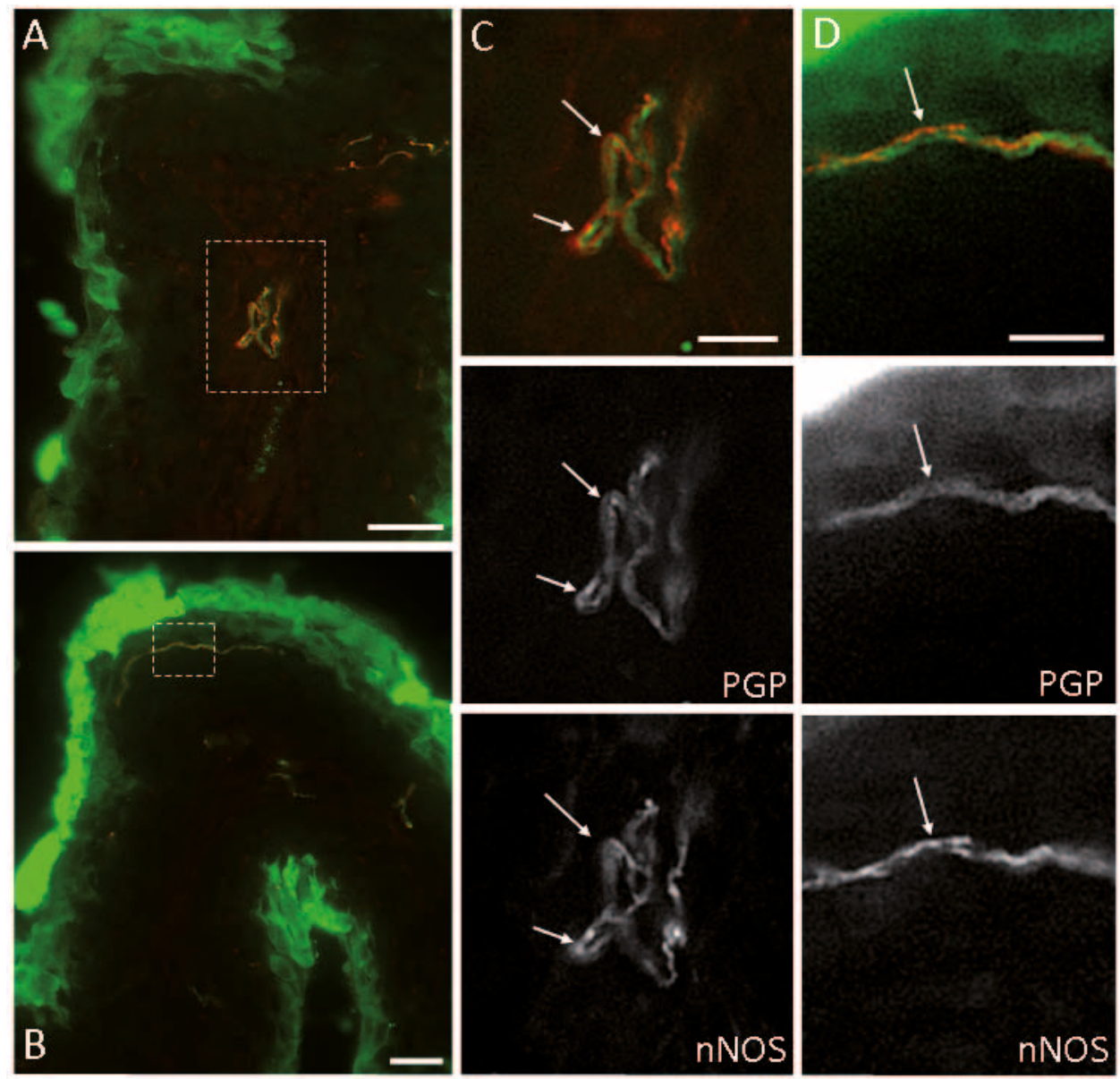

Figure 6. Photomicrographs from the suburothelium of the bladder. The section is double stained for PGP (green) and nNOS (red). The fibre shown in panel $\mathrm{A}$ is magnified in panel $\mathrm{C}$ and does not seem to co-localize (arrows). The fibre running right beneath the urothelium shown in Panel B is magnified in panel $D$ and does not show co-localization. Both fibres could be examples where ephaptic transmission could take place. Calibration bars:A and B; $50 \mu \mathrm{m}, \mathrm{C}$ and D I0 $\mu \mathrm{m}$. 

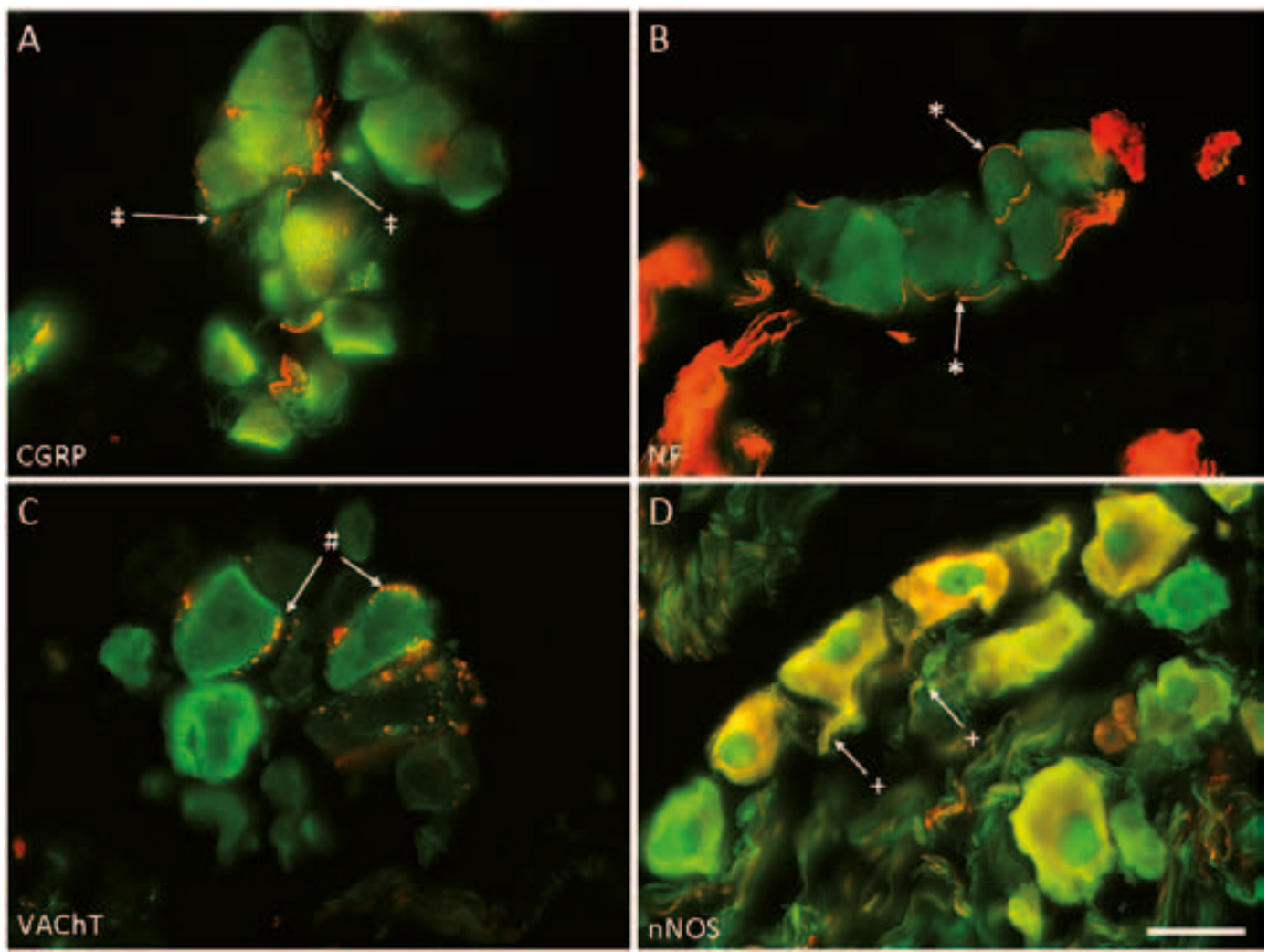

Figure 7. Ganglia on the serosal side of the bladder base. (A) CGRP-IR nerve fibres (red) in close proximity to several PGP-IR (green) ganglia, the arrows indicate possible nerve terminals. (B) NF-IR nerve fibres (red) can be seen to run between PGP-IR ganglia (green). (C)VAChT-IR varicosities (red) are seen in close to the ganglia possibly making contact. (D) nNOS-IR nerve fibres (red) co-localize with almost all the PGP-IR ganglia (green), some ganglia remain only PGP-IR. Large nerve bundles are seen running towards the bladder base and other ganglia. Note that ganglia can contain various numbers of nerve cell bodies. Calibration bar at $50 \mu \mathrm{m}$. 
Figure 8. Diagrammatic representation of the localization of nerve fibres stained by the four different antibodies in the mouse bladder. Panel A summarizes the situation in the bladder base. Panel $B$ summarizes the situation in the bladder lateral wall. Specific differences can be seen in both panel $A$ and $B, C G R P$ more densely innervates the suburothelial layer compared to the lateral wall, moreover CGRP innervation of the inner muscle is denser compared to the inner muscle in the bladder base.VAChT innervation is denser at the suburothelial layer at the bladder base compared to the lateral wall. No differences are seen between NF and nNOS when comparing bladder base and lateral wall.The circular dots represent nerve fibre endings.
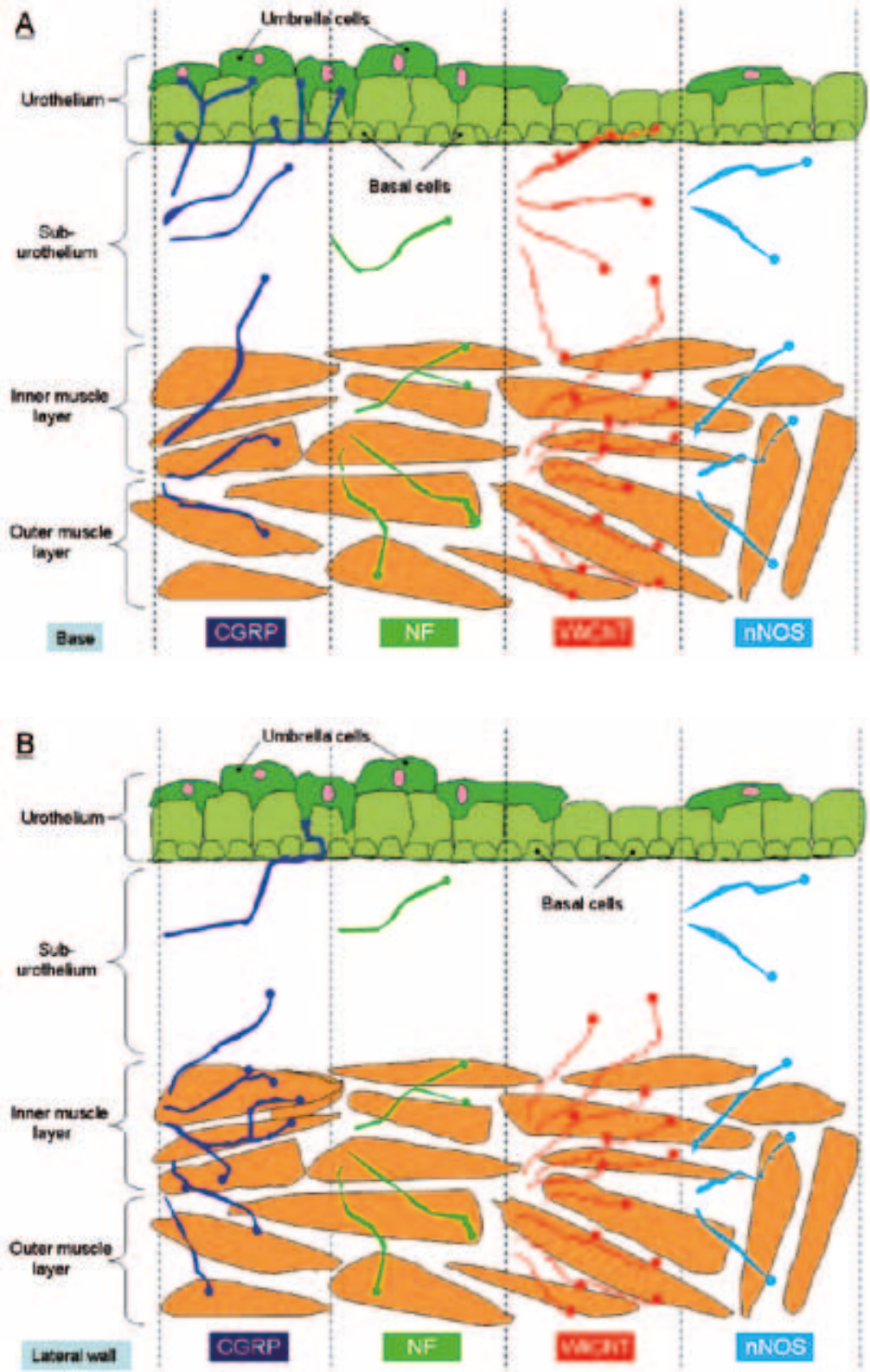
Ganglia were exclusively present on the serosal side at the bladder base. The population of ganglia was heterogeneous showing several different immunohistochemical staining patterns (Figure 7). Bundles of nerve fibres were seen running between the ganglia. CGRP and NF nerve fibres ran in proximity to the cell bodies. All neuronal cell bodies were PGP-IR, with some differences in staining intensity; most of these cell bodies were also nNOS-IR. None of the nerve cell bodies expressed CGRP-IR or NF-IR. $\mathrm{nNOS}-\mathrm{IR}$ and VAChT-IR were also visile running between the ganglia. Clusters were small, ranging from I or 2 up to 15 nerve cell bodies per ganglion.

\section{Discussion}

In this study we observed a heterogeneous immunohistochemical staining pattern for the afferent nerve fibre which might reflect a functional differentiation. Afferent nerve staining pattern and distribution within the bladder wall is schematically depicted in Figure 8. Furthermore, regional differences between the lateral wall and bladder base are shown.

CGRP-IR axonal nerve fibres endings originate at several locations in the urothelium including the basal layer, the urothelial epithelial cells and even right underneath the umbrella cells, characterizing them as primary afferent neurons. These nerve fibres are likely to be afferent $\mathrm{C}$-fibres. These fibres have been shown to be responsive to capsaicin, releasing CGRP into the bladder wall, after activation of the TRPVI receptor ". Although the cellular location of the TRPVI receptor in the bladder is still under debate, but recent literature has shown colocalization with CGRP indicating it's presence on afferent nerve fibres extending into the urothelium ${ }^{12}$. The most likely location is in the urothelial cells ${ }^{13}$. Because CGRP has been found to inhibit the phasic activity in the isolated bladder preparation these fibres could be part of a motor/sensory system ${ }^{14}$. This is emphasized by the fact that deletion of the TRPVI receptor in knock out mice resulted in diminished afferent responses to mechanical stimuli ${ }^{15}$. The lamina propria at bladder base was more densely innervated by these CGRP-IR afferent nerve fibres than the lateral wall, similar observations have been described in the guinea pig and the rat urinary bladder, which suggests that this region has an important sensory function ${ }^{5,16,17 .}$ At the lateral wall, the inner muscle layer was densely innervated by CGRP-IR nerve fibres, in contrast to the outer muscle layer. This difference in innervation of the muscle layers was not observed at the bladder base (Figure I). These CGRP-IR fibres in the inner muscle layer of the lateral wall could be mechanosenstive nerve fibres responding to stretch of the bladder wall, as the lateral wall is significantly stretched during bladder filling and phasic contractions ${ }^{18}$. Indeed in the rat Shea et al. demonstrated that administration of capsaicin elicits activity in most of the mechanosenstive nerve fibres ${ }^{2}$. Furthermore, in the mouse bladder functional research also identifies stretchresponsive muscular and muscular/urothelial fibres at the lateral wall ${ }^{8}$. Since most of the PGP-IR and CGRP-IR nerve fibres ran close to each other in the sub-urothelial space, there is a possibility of direct exchange of impulses between the nerve fibres. Two mechanisms illustrate the possible direct exchange of information between nerve fibres, e.g. ephaptic transmission and transmission via axo- 
Structural and Functional Aspects of Sensory-motor Interaction in the Urinary Bladder

axonal synapses (Figure 2, panel (b)). In 1942 Arvanitaki proposed the term; "ephaptic transmission" indicating signal transfer purely by electrical impulses between adjacent membranes of nerve fibres as opposed to synaptic transmission at sites of specialized junctions ${ }^{19}$. This transmission via axo-axonal synapses uses neurotransmitters for excitation or inhibition of adjacent nerve fibres ${ }^{20}$. In the bladder a pre-junctional synaptic interaction has been shown between cholinergic and adrenergic nerves mediated via inhibitory muscarinic receptors on adrenergic nerve terminals ${ }^{21}$ In the CNS a comparable interaction has been identified, where synapses play a role in the sensory-motor integration of an ongoing motor task ${ }^{22}$. Future studies using synaptophysin could possibly show a structural relationship between different nerve fibre types, and detect additional pre-junctional signal modulation systems within the bladder wall ${ }^{23}$. As a specific note we observed that some nerve fibres were not PGP-IR suggesting that PGP might not be a universal marker for nerve fibres. This observation was supported by another PGP $\mathrm{Ab}$, which gave similar results. This possibly indicated two different afferent nerve fibres, one PGP-IR positive and PGP-IR negative nerve fibre. NF-IR nerve fibres were primarily located in the muscle, with few fibre endings running in the lamina propria, these observations are consistent with observations in the guinea pig bladder ${ }^{6}$. NF-IR nerve fibres were not distributed throughout all layers of the bladder wall. In this study NF is used as an marker for A $\delta$ nerve fibres since in the dorsal root ganglia (DRG) NF is exclusively expressed in myelinated $A \delta$-fibres, and not in unmyelinated C-fibres ${ }^{10}$. However, the majority of bladder afferent nerve fibres is NF-negative ${ }^{24}$. In the muscle layers, these fibres are likely to be mechanosenstive responding to the slow filling of the bladder ${ }^{25}$. On the serosal side at the bladder base, both CGRP-IR afferent and NF-IR afferent axons probably project to ganglia were there signals are modulated and re-directed ${ }^{6}$. Another class of afferent fibre running near the urothelium were the VAChT-IR, cholinergic fibres, running close to the basal cell and the urothelial epithelial cell layers (Figure 5). As with all nerve fibres running near the urothelium, VAChT-IR nerve fibres were considered to be afferent: as hypothesis proposed by Gosling and Dixon states that, "a sensory role can reasonably be proposed for those peripheral nerves whose terminals are regularly observed to be unrelated to recognized neuro-effector target sites" ${ }^{3}$. Moreover, in the rat, cholinergic nerve fibres in the lamina propria, expressing acetylcholine-esterase, have been found to co-localize with CGRP within the urothelium of ureters ${ }^{26}$. The lamina propria at the bladder base was more densely innervated with VAChT-IR nerve fibres, in parallel with the distribution of CGRP-IR nerve fibres. These regional variations in innervation of the bladder base and the lateral wall have been described in the rats and in humans as well ${ }^{16,27}$. The muscle layers were uniformly innervated by VAChT-IR nerve fibres, where this marker could identify both afferent and efferent fibres ${ }^{28,29}$. Nerve fibres containing nNOS were distributed throughout the bladder but predominated in the bladder base. Most NOS-containing (nitrergic) nerve fibres also contain VAChT and can therefore be considered cholinergic ${ }^{30}$. These nerve fibres were primarily found in the muscle layers with few nerve fibres running in the suburothelial layer. Dixon et al. have shown that nNOS is sparse 
in cholinergic nerves of the lateral wall, but present in the majority of these nerves at the bladder base ${ }^{27}$. In the muscle the bulk of cholinergic fibres are presumed to be efferent motor neurons responsible for initiating and sustaining a detrusor contraction ${ }^{29}$. At the bladder base cholinergic fibres may release NO known to induce smooth muscle relaxation in the urethra by activating guanylate cyclase and increasing cyclic guanosine monophosphate (cGMP) levels in the smooth muscle cell ${ }^{31}$. The latter observation may reflect a neurotransmitter mediated mechanism preceding micturition. Cholinergic stimulation gives rise to relaxation of smooth muscle at the bladder neck as well as a contraction of the detrusor body ${ }^{17}$. The function of the nNOS nerve fibres found in the lateral wall is less clear since not detrusor smooth muscle cells but interstitial cells have been seen to produce cGMP in response to NO ${ }^{32,33}$. The role of these cells is still uncertain possibly playing a role in the generation of non voiding activity seen during bladder filling ${ }^{32}$. Ganglia were seen at the serosal side of the bladder; all were PGP-IR and most were also nNOS-IR. Varicosities of CGRP-IR and VAChT-IR nerve fibres, possibly indicating nerve terminals, and NF-IR nerve fibres were running in close proximity to the ganglia (Figure 7). These ganglia could be relay stations transferring input received from afferent nerve fibres in the bladder to the CNS as suggested by similar observations in the guinea pig bladder and also seen in other organ systems ${ }^{6,34}$. In addition, ganglia could also function in local motor-sensory networks of the bladder. Efferent output of these ganglia could be directed towards the interstitial cells or the smooth muscle cells. Together with the urothelium, these structures might play a role in the generation and modulation of non voiding activity seen during bladder filling ${ }^{18,35}$. Indeed, activating CGRP-IR afferent nerve fibres decreases non voiding activity by inducing a smooth muscle response ${ }^{14}$. This response could be mediated by ganglia receiving afferent input from CGRP-IR nerve fibres. As the inhibition is more pronounced during rapid emptying these CGRP-IR nerve fibres could be activated by the release of NO or ATP from the urothelium via the TRPVI receptor ${ }^{36,37}$. On the other hand adding $\mathrm{NO}$ to the isolated bladder preparation increased this non voiding activity ${ }^{38}$. The mechanism of action is unclear but may involve cGMP produced by interstitial cells and cell in the sub-urothelial layer in response to $\mathrm{NO}$. In the lamina propria $\mathrm{NO}$ may inhibit the excitability of bladder afferents, increasing the micturition threshold and decreasing the micturition frequency ${ }^{39}$. Alternatively, non voiding activity can be increased by infusion of volume in the bladder, activating suburothelial cholinergic nerve fibres or suburothelial interstitial cells via the $M 3$ receptor by the release of $A C h$ from the urothelium ${ }^{40}$. This could induce a release of ACh from VAChT-IR nerve fibres at the ganglia initiating a response to the detrusor via motor nerve fibres. Non voiding activity could be important in the generation of sensation as rapid stretch of the bladder wall produces afferent discharge of mechanosensitive stretch receptors ${ }^{7,38}$. Indeed, during normal functioning, the bladder is continuously bombarded by a vast amount of stimuli, potentially generating and enormous amount of afferent impulses to the CNS, however not all impulses leaving the urinary bladder elicit conscious sensation ${ }^{7}$. This 'afferent noise' is generated by different afferent nerve fibres, of which some are described in this 
study. In summary, using immunohistochemistry several sub populations of afferent nerve fibres were recognized in the mouse bladder and regional differences were identified. Based on our working hypothesis, that all nerve fibres in the lamina propria are considered to be afferent, we found at least four different types of afferent nerve fibre; CGRP-IR, NF-IR, VAChT-IR and nNOS-IR. At the bladder base the suburothelium was densely innervated by all nerve fibres types, indicating that bladder sensation is predominant at this location. The dense innervation of CGRP-IR nerve fibres in the inner muscle at the lateral wall implicates a possible mechanosensitive role for these nerve fibres at this location. The NF-IR nerve fibres are probably also mechanosensitive, as they were primarily found in the muscle layers. The majority of VAChT-IR nerve fibres in the muscle will most likely be efferent nerve fibres responsible for the detrusor contraction. At the bladder base nNOS-IR nerve fibres will most likely induce smooth muscle relaxation whereas nNOS-IR nerve fibres found at the lateral wall probably modulate the activity of interstitial cells. The ganglia located at the serosal side of the bladder were all PGP-IR and mostly nNOS-IR. All nerve fibres types ran in close proximity suggesting that these ganglia function as local motor-sensory networks receiving input from multiple different afferent nerve fibres and directing efferent output to the CNS and the bladder wall (figure 8). Further research breaching between functional and structural research is paramount to gain insight into the role of these different afferent nerve fibres during normal bladder functioning and in relation to bladder pathology, such as the overactive bladder syndrome. 


\section{References}

I. Fowler, C.J., Griffiths, D., de Groat,W. C.:The neural control of micturition. Nat Rev Neurosci, 9:453, 2008

2. Shea, V. K., Cai, R., Crepps, B. et al.: Sensory fibers of the pelvic nerve innervating the Rat's urinary bladder. J Neurophysiol, 84: 1924, 2000

3. Gosling, J. A., Dixon, J. S.: Sensory nerves in the mammalian urinary tract. An evaluation using light and electron microscopy. J Anat, II7: 133, 1974

4. Andersson, K. E.: Bladder activation: afferent mechanisms. Urology, 59: 43, 2002

5. Grol, S., van Koeveringe, G. A., de Vente, J. et al.: Regional differences in sensory innervation and suburothelial interstitial cells in the bladder neck and urethra. BJU Int, 2008

6. Gillespie, J. I., Markerink-van Ittersum, M., de Vente, J.: Sensory collaterals, intramural ganglia and motor nerves in the guinea-pig bladder: evidence for intramural neural circuits. Cell Tissue Res, 325: 33, 2006

7. Gillespie, J. I., van Koeveringe, G. A., de Wachter, S. G. et al.: On the origins of the sensory output from the bladder: the concept of afferent noise. BJU Int, 103: 1324, 2009

8. Xu, L., Gebhart, G. F.: Characterization of mouse lumbar splanchnic and pelvic nerve urinary bladder mechanosensory afferents. J Neurophysiol, 99: 244, 2008

9. Lagou, M., De Vente, J., Kirkwood, T. B. et al.: Location of interstitial cells and neurotransmitters in the mouse bladder. BJU Int, 97: 1332, 2006

10. Lawson, S. N., Perry, M. J., Prabhakar, E. et al.: Primary sensory neurones: neurofilament, neuropeptides, and conduction velocity. Brain Res Bull, 30: 239, 1993

II. Maggi, C. A., Lecci, A., Santicioli, P. et al.: Cyclophosphamide-induced cystitis in rats: involvement of capsaicin-sensitive primary afferents. Agents Actions, $38 \mathrm{Spec}$ No: C28, 1993

12. Yu, W., Hill, W. G., Apodaca, G. et al.: Expression and distribution of transient receptor potential (TRP) channels in bladder epithelium. Am J Physiol Renal Physiol, 300: F49

13. Birder, L. A., Kanai, A. J., de Groat, W. C. et al.: Vanilloid receptor expression suggests a sensory role for urinary bladder epithelial cells. Proc Natl Acad Sci U S A, 98: 13396, 200 I

14. Gillespie, J. I.: Inhibitory actions of calcitonin gene-related peptide and capsaicin: evidence for local axonal reflexes in the bladder wall. BJU Int, 95: 149, 2005

15. Daly, D., Rong, W., Chess-Williams, R. et al.: Bladder afferent sensitivity in wild-type and TRPVI knockout mice. J Physiol, 583: 663, 2007

16. Mohammed, H. A., Santer, R. M.: Distribution and changes with age of calcitonin gene-related peptideand substance P-immunoreactive nerves of the rat urinary bladder and lumbosacral sensory neurons. Eur J Morphol, 40: 293, 2002

17. Zhou, Y., Ling, E. A.: Colocalization of nitric oxide synthase and some neurotransmitters in the intramural ganglia of the guinea pig urinary bladder. J Comp Neurol, 394: 496, 1998

18. Gillespie, J. I., Harvey, I. J., Drake, M. J.: Agonist- and nerve-induced phasic activity in the isolated whole bladder of the guinea pig: evidence for two types of bladder activity. Exp Physiol, 88: 343, 2003

19. Arvanitaki, A.: Effects evoked in an axon by the activity of a contiguous one. Journal of Neurophysiology, 5: 89, 1942 
Structural and Functional Aspects of Sensory-motor Interaction in the Urinary Bladder

20. Gray, E. G.: Electron microscopy of excitatory and inhibitory synapses: a brief review. Prog Brain Res, 31: 14I, 1969

21. Mattiasson, A., Andersson, K. E., Elbadawi, A. et al.: Interaction between adrenergic and cholinergic nerve terminals in the urinary bladder of rabbit, cat and man. J Urol, 137: 1017, 1987

22. Clarac, F., el Manira, A., Cattaert, D.: Presynaptic control as a mechanism of sensory-motor integration. Curr Opin Neurobiol, 2: 764, 1992

23. Rasminsky, M.: Ephaptic transmission between single nerve fibres in the spinal nerve roots of dystrophic mice. J Physiol, 305: I5I, 1980

24. Yoshimura, N., Erdman, S. L., Snider, M. W. et al.: Effects of spinal cord injury on neurofilament immunoreactivity and capsaicin sensitivity in rat dorsal root ganglion neurons innervating the urinary bladder. Neuroscience, 83: 633, 1998

25. Janig, W., Morrison, J. F.: Functional properties of spinal visceral afferents supplying abdominal and pelvic organs, with special emphasis on visceral nociception. Prog Brain Res, 67: 87, 1986

26. Sann, H., McCarthy, P. W., Mader, M. et al.: Choline acetyltransferase-like immunoreactivity in small diameter neurones of the rat dorsal root ganglion. Neurosci Lett, 198: 17, 1995

27. Dixon, J. S., Jen, P. Y., Gosling, J. A.: The distribution of vesicular acetylcholine transporter in the human male genitourinary organs and its co-localization with neuropeptide $Y$ and nitric oxide synthase. Neurourol Urodyn, 19: 185, 2000

28. Nandigama, R., Bonitz, M., Papadakis, T. et al.: Muscarinic acetylcholine receptor subtypes expressed by mouse bladder afferent neurons. Neuroscience, 168: 842

29. Andersson, K. E., Hedlund, P.: Pharmacologic perspective on the physiology of the lower urinary tract. Urology, 60: 13, 2002

30. Andersson, K. E., Wein, A. J.: Pharmacology of the lower urinary tract: basis for current and future treatments of urinary incontinence. Pharmacol Rev, 56: 581, 2004

31. Andersson, K. E., Persson, K.: Nitric oxide synthase and the lower urinary tract: possible implications for physiology and pathophysiology. Scand J Urol Nephrol Suppl, 175: 43, 1995

32. Gillespie, J. I., Drake, M. J.: The actions of sodium nitroprusside and the phosphodiesterase inhibitor dipyridamole on phasic activity in the isolated guinea-pig bladder. BJU Int, 93: 85I, 2004

33. Smet, P. J., Jonavicius, J., Marshall, V. R. et al.: Distribution of nitric oxide synthase-immunoreactive nerves and identification of the cellular targets of nitric oxide in guinea-pig and human urinary bladder by cGMP immunohistochemistry. Neuroscience, 7I: 337, 1996

34. Hardwick, J. C., Mawe, G. M., Parsons, R. L.: Evidence for afferent fiber innervation of parasympathetic neurons of the guinea-pig cardiac ganglion. J Auton Nerv Syst, 53: 166, 1995

35. Kanai, A., Roppolo, J., Ikeda, Y. et al.: Origin of spontaneous activity in neonatal and adult rat bladders and its enhancement by stretch and muscarinic agonists. Am J Physiol Renal Physiol, 292: FI065, 2007

36. Avelino, A., Cruz, F.: TRPVI (vanilloid receptor) in the urinary tract: expression, function and clinical applications. Naunyn Schmiedebergs Arch Pharmacol, 373: 287, 2006 
37. Birder, L. A., de Groat, W. C.: Mechanisms of disease: involvement of the urothelium in bladder dysfunction. Nat Clin Pract Urol, 4: 46, 2007

38. McCarthy, C. J., Zabbarova, I. V., Brumovsky, P. R. et al.: Spontaneous contractions evoke afferent nerve firing in mouse bladders with detrusor overactivity. J Urol, I8I: 1459, 2009

39. Persson, K., Igawa, Y., Mattiasson, A. et al.: Effects of inhibition of the L-arginine/nitric oxide pathway in the rat lower urinary tract in vivo and in vitro. Br J Pharmacol, 107: 178, 1992

40. Grol, S., Essers, P. B., van Koeveringe, G. A. et al.: M(3) muscarinic receptor expression on suburothelial interstitial cells. BJU Int, 104: 398, 2009 

Chapter 4

\section{Non-voiding Activity of the Guinea Pig Urinary Bladder}

Bart T. Biallosterski, Gommert A. v Koeveringe, Philip E. v Kerrebroeck, James I. Gillespie and Stefan G. de Wachter

PMID: 21683402

Journal of Urology 201I 
Structural and Functional Aspects of Sensory-motor Interaction in the Urinary Bladder

\begin{abstract}
Purpose

Non voiding contractions (NVCs) of the detrusor occur in up to $70 \%$ of healthy individuals. These contractions increase in pathological detrusor function and may be associated with afferent activity. We examine NVCs in the urethane-anaesthetized guinea pig bladder and study the effect of filling rate and intravesical volume.
\end{abstract}

\title{
Materials and Methods
}

I4 guinea pigs were anesthetized and the bladder catheterised in the dome. In 6 guinea pigs bladder infusion was continuous, two physiological filling rates of $25 \mu \mathrm{L} / \mathrm{min}(0,75 \mathrm{HD})$ and $50 \mu \mathrm{L} / \mathrm{min}(\mathrm{I}, 5$ HD) were used. In 8 additional guinea pigs isovolumetric cystometry was performed filling the bladder with increments, recording at low, medium and high intravesical volume.

Results

NVCs were apparent in all animals. Contractions increased in frequency and amplitude as the bladder filled. Different phases were identified, immediately after a void no NVCs were observed followed by continuous activity, first with small contractions and later with both small and large contractions. Small NVCs show a phasic pattern in frequency, while the frequency of large NVCs either slowly increases or remains stable. Frequency and amplitude of NVCs were higher with faster filling rate and higher intravesical volume.

\section{Conclusions}

NVCs are present in the anaesthetized guinea pig. Under normal physiological conditions NVCs increase in amplitude and frequency with the increase in filling rate and intravesical volume. Small and large NVCs differ in frequency pattern and occur at different periods of bladder filling. This may illustrate different afferents functioning during bladder filling, which could be important in pathology. 


\section{Introduction}

For decades, the bladder was believed to be "silent", e.g. without any transient pressure increases during the filling phase, and presence of detrusor overactivity was considered to be pathological. These involuntary detrusor contractions may lead to symptoms such as urgency, increased frequency of voiding and incontinence, which seriously affect the quality of life and ability to work. However ambulatory urodynamics has shown non voiding detrusor contractions during the filling phase in up to $69 \%$ of healthy volunteers ${ }^{1,2}$, suggesting that these contractions may be a normal physiological mechanism. Currently, the differences in non voiding contractions (NVCs) between normal and pathological states are unknown. These NVCs have been shown to occur in vitro in the isolated whole bladder preparation ${ }^{3}$, and in that model, the NVC's can be increased by ATP, NO, Acetylcholine and bladder volume or decreased by PDEs and CGRP ${ }^{4-9}$. These data suggest that the NVCs are generated and processed at least in part within the bladder wall itself. In rats, the NVC have been studied in vivo, showing that they increase in amplitude and frequency as the bladder fills $^{10}$. The exact role or purpose of the NVCs is still unknown, but if they are able to induce afferent activity, they could serve as a "primary sensory mechanism" within the bladder. Enhancement of this activity might then result in increased bladder sensations leading to urgency ". The current study evaluates and describes NVCs in the bladder of the anaesthetized guinea pig. Furthermore, the effect of different filling rates and the effect of intravesical volume is investigated.

\section{Materials and Methods}

All experimental procedures were approved by the local ethical committee of the Maastricht University for animal experiments according to the Dutch governmental guidelines. In total I4 male Hartley guinea pigs (294 g. \pm 46 SD, Charles River Laboratories) were used to perform in vivo cystometry. The guinea pigs were anesthetized with a solution of urethane $(20 \% \mathrm{w} / \mathrm{v}$ in saline $\mathrm{pH} 7,4 ;$ I,5 g./kg i.p.) given as a single dose. The guinea pig was placed supine on a heated pad to maintain body temperature and the bladder was exposed via a low midline abdominal incision. Two polyethylene-50 catheters (I.D. 0,58 mm O.D. 0,965 mm, PE-50, Becton Dickinson, Franklin Lakes, $\mathrm{NJ}$, USA) were passed through a small opening at the apex of the bladder dome and secured with a purse string suture (5-0 monocryl, Ethicon Inc., Somerville, New Jersey, USA). One catheter was connected to an infusion pump (Vickers Medical, Treonic IP4 syringe pump, Basingstoke, England) and the other was connected to a pressure transducer (DTX Plus, Becton Dickinson, Franklin Lakes, NJ, USA).The transducer output was amplified, digitized at $200 \mathrm{~Hz}$ and recorded using a data capture system (Acqknowledge version 3.7.3, Biopac systems, Inc., Goleta, USA). The pressure range of this apparatus was $0,02-180 \mathrm{cmH}_{2} \mathrm{O}$. The transducer was calibrated before each experiment. A 30 minute rest period was included before starting bladder filling. Then the bladder was filled continuously and two filling and voiding cycles were recorded before the experimental protocols were started. A NVC was considered any increase in detrusor pressure without urine leakage, which was visually recorded. 
In part I, the effect of different filling rates on the development of NVCs was studied in 6 animals. Saline was continuously infused at room temperature, and three voiding cycles were recorded. During bladder filling, the amplitude and time at peak amplitude were determined for every NVC. Since no data are available on the physiological urine output in the guinea pig, we based the filling rate on data from the rat [12], filling the bladder at two physiological rates; $25 \mu \mathrm{L} / \mathrm{min}(0,75 \mathrm{HD})$ and $50 \mu \mathrm{L} / \mathrm{min}(\mathrm{I}, 5 \mathrm{HD})$, used in the same guinea pig.

In part 2, the effect of bladder distension on the development of NVCs was studied in 8 animals during isovolumetric conditions. Before the start of the isovolumetric measurements, the volume at which the recordings were made was determined by the micturition volume threshold as measured during two consecutive fillings at $25 \mu \mathrm{L} / \mathrm{min}$. Isovolumetric volumes were defined as 25 (low), 50 (medium) and $75 \%$ (high) of the micturition volume threshold. Intravesical pressure was then recorded for at least 10 minutes at each predefined volume. Natural diuresis was considered to be negligible as i.p. urethane dramatically decreases urine production ${ }^{13}$, which was also supported by the observations that basal bladder pressure did not change during the isovolumetric recordings.

\section{Data analysis}

Paired-samples t-tests were used for analysis with $\mathrm{P}<0.05$ set as level of significance for paired filling and for isovolumetric data (SPSS, version I5, Armonk, New York, USA).

\section{Results}

\section{Part I Continuous bladder filling}

During the continuous bladder filling, the filling phase can be divided into two phases; a 'silent' phase and an 'activity' phase (Figure IA). These phases were observed both with the slow and fast filling rates. The 'silent' phase is characterised by the absence of NVCs (Figure I, 'silent phase', inset I in (A) and (B)). At the end of the 'silent' phase, the 'activity' phase starts with NVCs occurring at irregular intervals. These NVCs then become larger and more regular, increasing in amplitude (Figure I,'activity phase', insets I, 2 and 3 in both (A) and (B)).The filling phase ends with a fast and large voiding contraction (Figure I). 
A

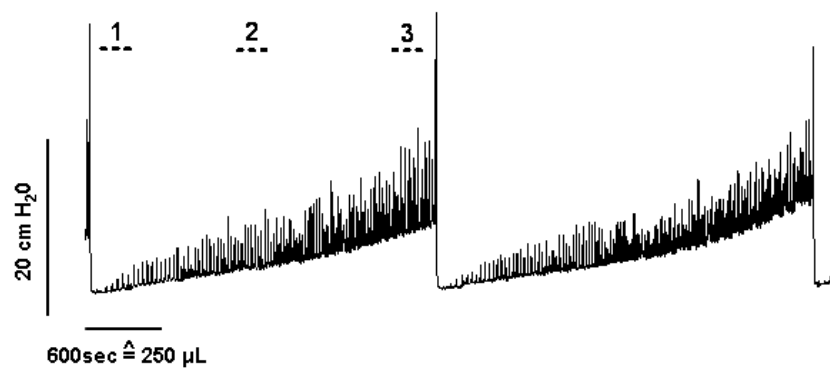

1

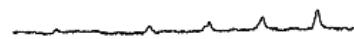

'Lumaldan'

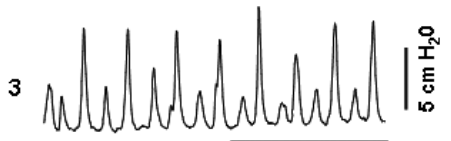

$120 \mathrm{sec} \hat{=} 50 \mu \mathrm{L}$

\section{B}
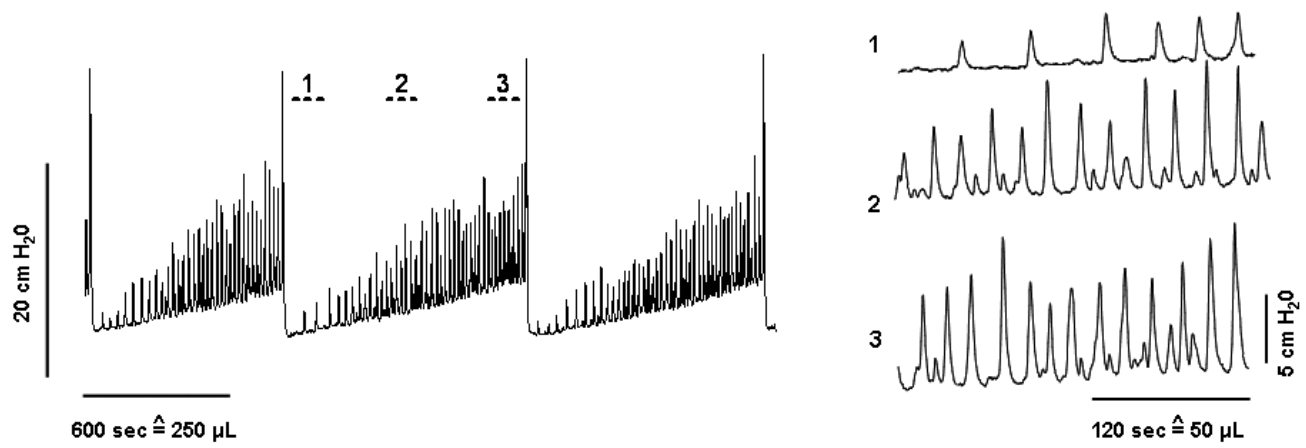

Figure I. A, continuous filling cycles at slow filling rate $(25 \mu \mathrm{L} / \mathrm{min})$. Superimposed upon the gradual increasing basal pressure one can clearly distinguish the non voiding contractions occurring during the filling cycles. Insets I, 2, 3 illustrate sequential time periods throughout a fill, emphasizing the rise in amplitude and frequency of NVCs. B, 4 filling cycles in the same animal at a fast filling rate $(50 \mu \mathrm{L} / \mathrm{min})$. Filling time is significantly shorter and NVC amplitude is significantly increased (see insets). 
A
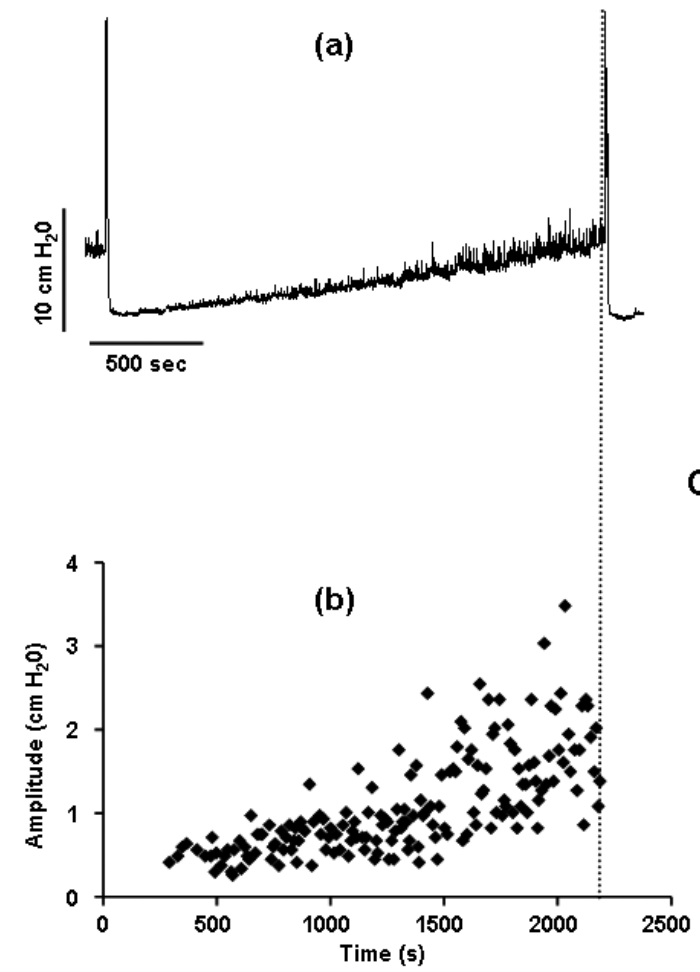

B

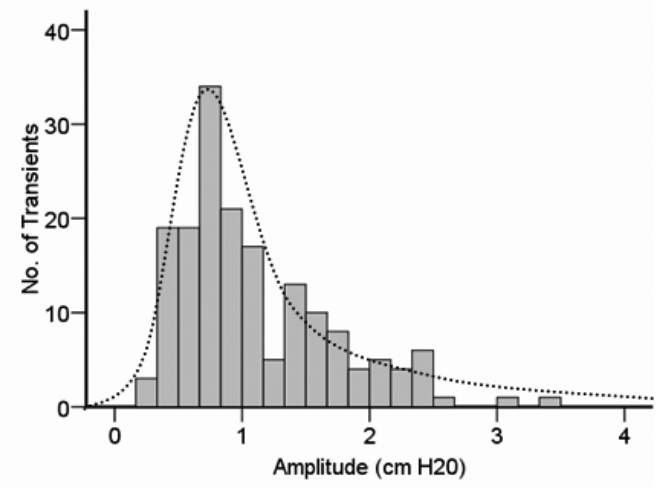

C

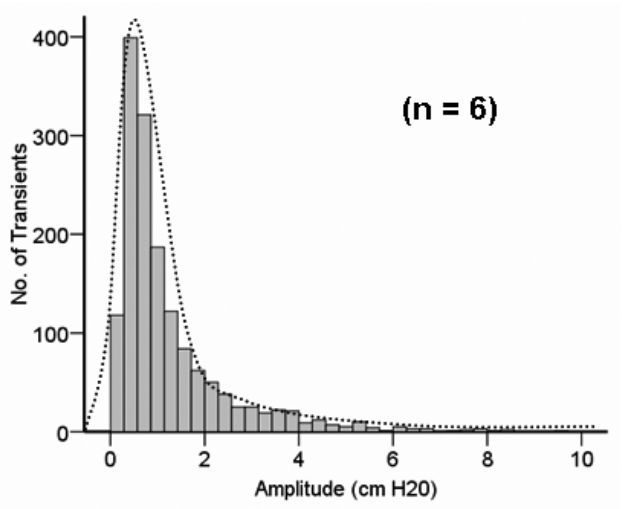

Figure 2. Amplitude analysis of the NVCs at slow filling rate. A, raw data (a) and amplitude distribution scatter plot (b) relating the increase in amplitude to the filling time. In the beginning of a cycle primarily small NVCs are present, towards the end of a fill this distribution changes, with the presence of larger NVCs. The dotted line shows the end of the fill, initiated by the start of micturition. B, NVC histogram (same guinea pig), C, NVC histogram of pooled data from 6 guinea pigs. Ordinates represent the number of transients at specific amplitude; the abscissa denotes the amplitude of the transients. 
Figure 2 illustrates the distribution of the amplitude of NVCs at a slow, physiological filling rate (25 $\mu \mathrm{L} / \mathrm{min})$. NVCs increase in amplitude when intravesical volume increases (Figure 2A, (b)). An amplitude distribution histogram is shown in fig. $2 \mathrm{~B}$ and shows that NVC amplitude is not normally distributed (median 0,9 $\pm 0,60 \mathrm{SD}$ ). This observation was consistent in all animals (Figure $2 \mathrm{C}, \mathrm{n}=6$, median 0,8 \pm I,25 SD, $\mathrm{p}<0,0 \mathrm{I}$, Shapiro-Wilk's W normality test).

From the start of the activity phase to the end the average increase in frequency of the NVC is approximately 3 fold as the bladder fills (ratio = 3,33 $\pm 3,48$ SD). Figure 3 shows the frequency distribution of the NVCs during bladder filling with the slow filling rate. On top of an overall gradual increase in frequency episodes with increased NVC activity can be distinguished, whereby the NVCs frequency increases and the NVC amplitude decreases (Figure 3A, marked). This phasic pattern was identified in all animals during all bladder filling phases with the two different filling rates. To study this pattern more in detail the population of NVC was split based on the median amplitude into small and large NVC (Figure $3 \mathrm{C}$ and D). The instantaneous frequency of small NVC showed an undulating pattern, whereas the instantaneous frequency of large NVCs gradually increases over time (Figure 3, panel C and D, respectively).

The basal pressure rise during the slow and fast filling was not different (Table I, Figure 4, panel A). However during fast filling a difference in NVC amplitude was noted, being most pronounced towards the end of the fill phase (Figure 4, B). A comparison of the average instantaneous frequency of NVCs at slow and fast filling rates does not show any significant differences.

Table I. Urodynamic parameters of 6 guinea pigs.

\begin{tabular}{llll}
\hline Cystometry Parameters & Slow & Fast & P-value \\
\hline Infused volume at start of Act. phase $(\mu \mathrm{l} \pm \mathrm{SD})$ & $107,24 \pm 56,07$ & $124,63 \pm 80,83$ & 0,48 \\
Max. infused volume $(\mu \mathrm{l} \pm \mathrm{SD})$ & $1006,98 \pm 262,65$ & $1099,82 \pm 408,25$ & 0,06 \\
Micturition pressure $(\mathrm{cm} \mathrm{H} 20 \pm \mathrm{SD})$ & $24,19 \pm 4,40$ & $24,49 \pm 4,63$ & 0,75 \\
Micturition threshold pressure $(\mathrm{cm} \mathrm{H} 20 \pm \mathrm{SD})$ & $8,60 \pm 1,70$ & $8,37 \pm 1,84$ & 0,63 \\
Basal pressure increase $(\mathrm{cm} \mathrm{H} 20 \pm \mathrm{SD})$ & $5,30 \pm 1,38$ & $4,79 \pm 1,70$ & 0,27 \\
\hline
\end{tabular}


A
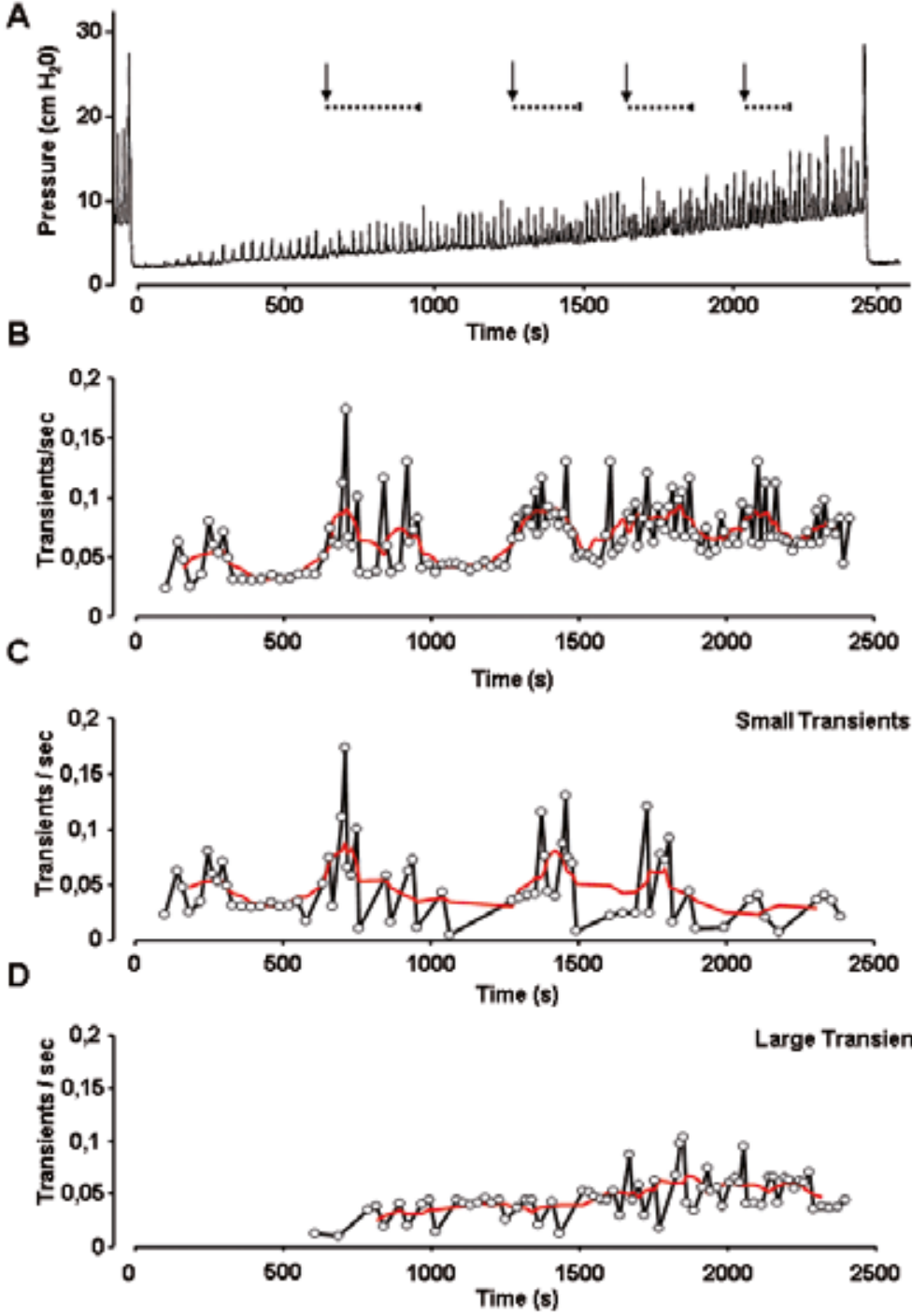

Figure 3. Frequency analysis of NVCs at slow filling rate.(A) Raw trace of slow filling rate NVCs are clearly present (see also Fig. IA). (B) Instantaneous frequency plot of the NVCs. Instantaneous frequency fluctuates and gradually increases during the fill. The red line indicates a moving average ( 7 consecutive NVCs) revealing a phasic pattern, indicating bursts of increasing frequency. Dotted lines in A indicate the increased number of NVCs (e.g. the increased frequency). B and C, the instantaneous frequency of small and large amplitude NVCs respectively. Red lines indicate the moving averages. The two groups were split according to the median $\left(0,89 \mathrm{~cm} \mathrm{H}_{2} 0\right)$. Phasic pattern within the instantaneous frequency are largely due to small NVCs, large NVCs underlie the slow rise in frequency. 
A
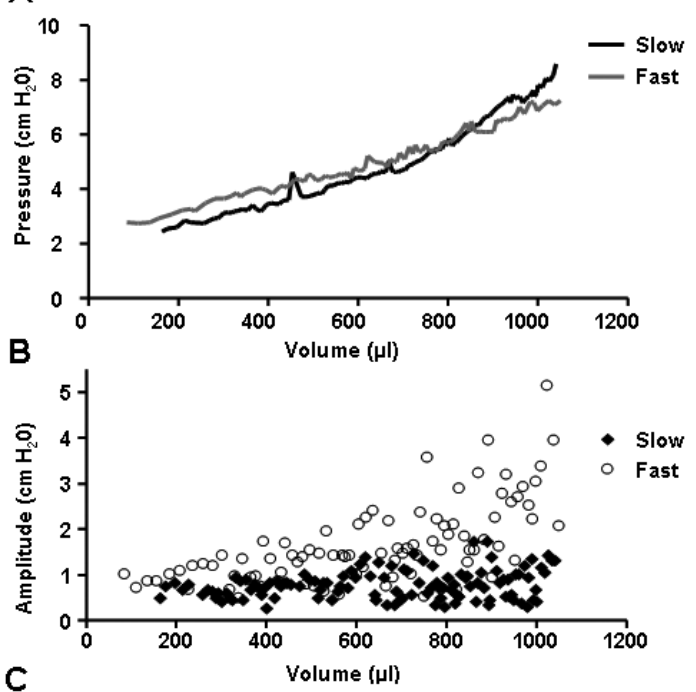

C

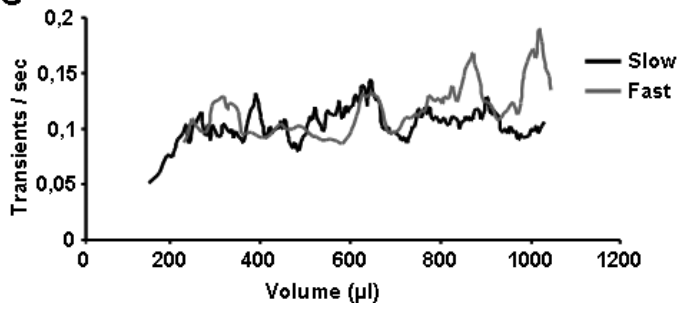

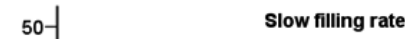

D
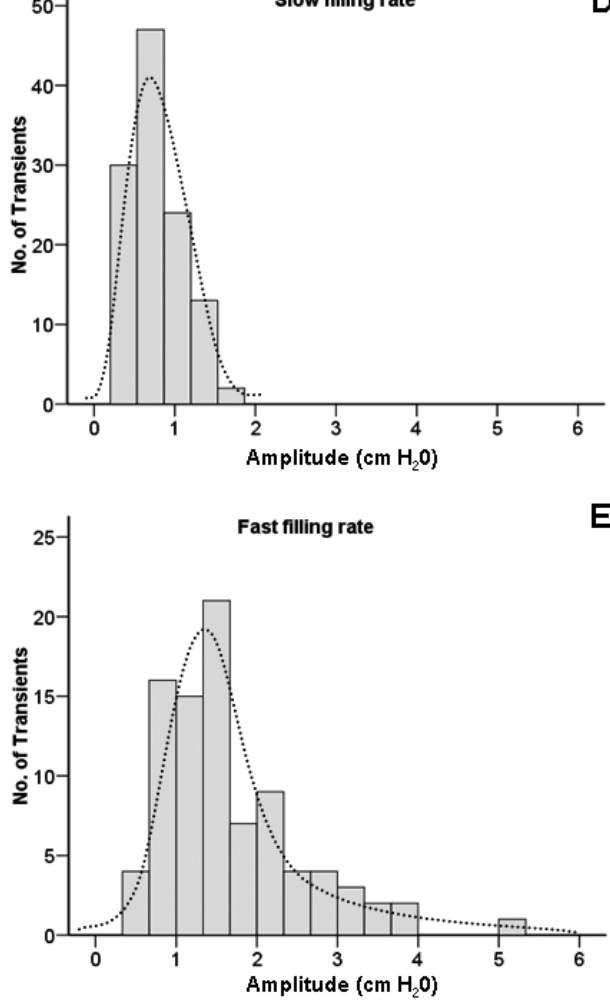

Figure 4. Effect of filling rate on NVCs in the same guinea pig. A, Basal pressure as a function of infused volume for slow and fast filling rates. B, NVC amplitude distribution scatter plot of the same two filling cycles. NVCs of the fast filling rate cycle (open circles) are bigger, especially towards the end of the fill, when compared to the slow filling rate (closed black circles). C, average instantaneous frequency. Towards the end of the fill the frequency of the NVCs in the fast rate filling increases more compared to the slow rate filling. D and E, NVC histograms of the slow and fast rate filling respectively.

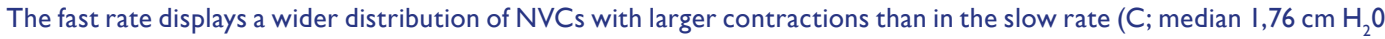
and D; median I,27 $\mathrm{cm} \mathrm{H}_{2} 0$ ) 
A

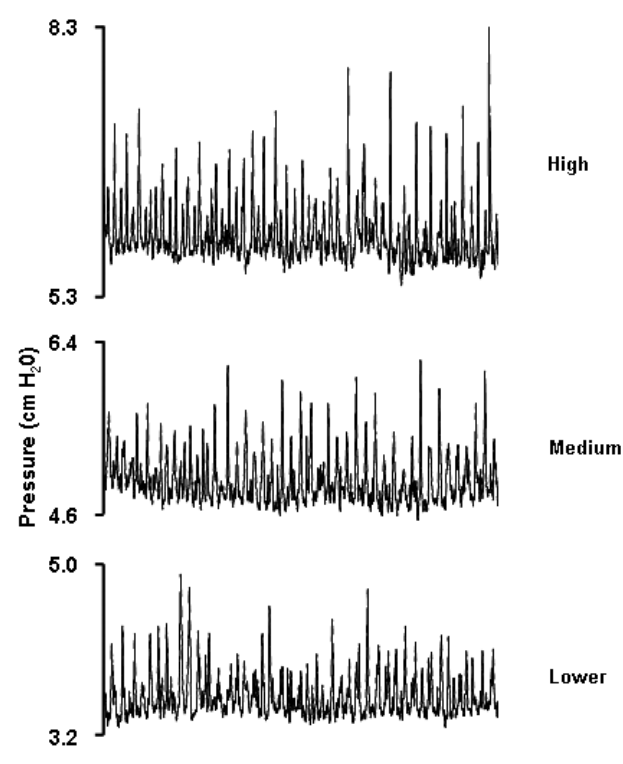

B

Av Amplitude

W. Av frequency

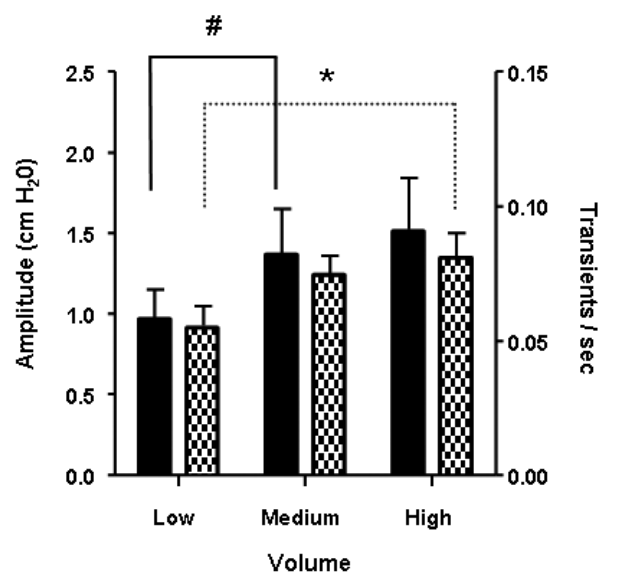

Figure 5. The effect of volume on NVCs.A, Isovolumetric recordings at low, medium and high intravesical volume. B, Bar chart comparing the NVCs at the three different volumes. Black bars indicate the rise in average amplitude (\# marker; low versus medium; $p=0,039)$. Dotted bars indicate the rise in instantaneous frequency $(*$ marker; low versus high; $p=0,025)$. The average basal pressure of the isovolumetric periods only reaches significant difference comparing low versus high $(n=8, p=0,009)$.

\section{Part 2, Isovolumetric recordings}

Bladder pressure was recorded at low, medium or high intravesical volume. Figure 5, panel A displays raw data of three isovolumetric conditions, showing that at higher volumes the NVC amplitude and frequency is significantly higher than at low volume (Figure 5, B). These data show that intravesical volume, apart from changes during filling, determines NVC amplitude and frequency. The average basal pressure of the isovolumetric periods only reaches significant difference comparing low versus high volume $(n=8, p=0.009)$. Furthermore, similar to the filling experiments, a phasic frequency pattern in the small NVC (< median amplitude) is observed at every volume and animal. The large NVCs have a constant frequency that does not increase over time (Figure 6). 

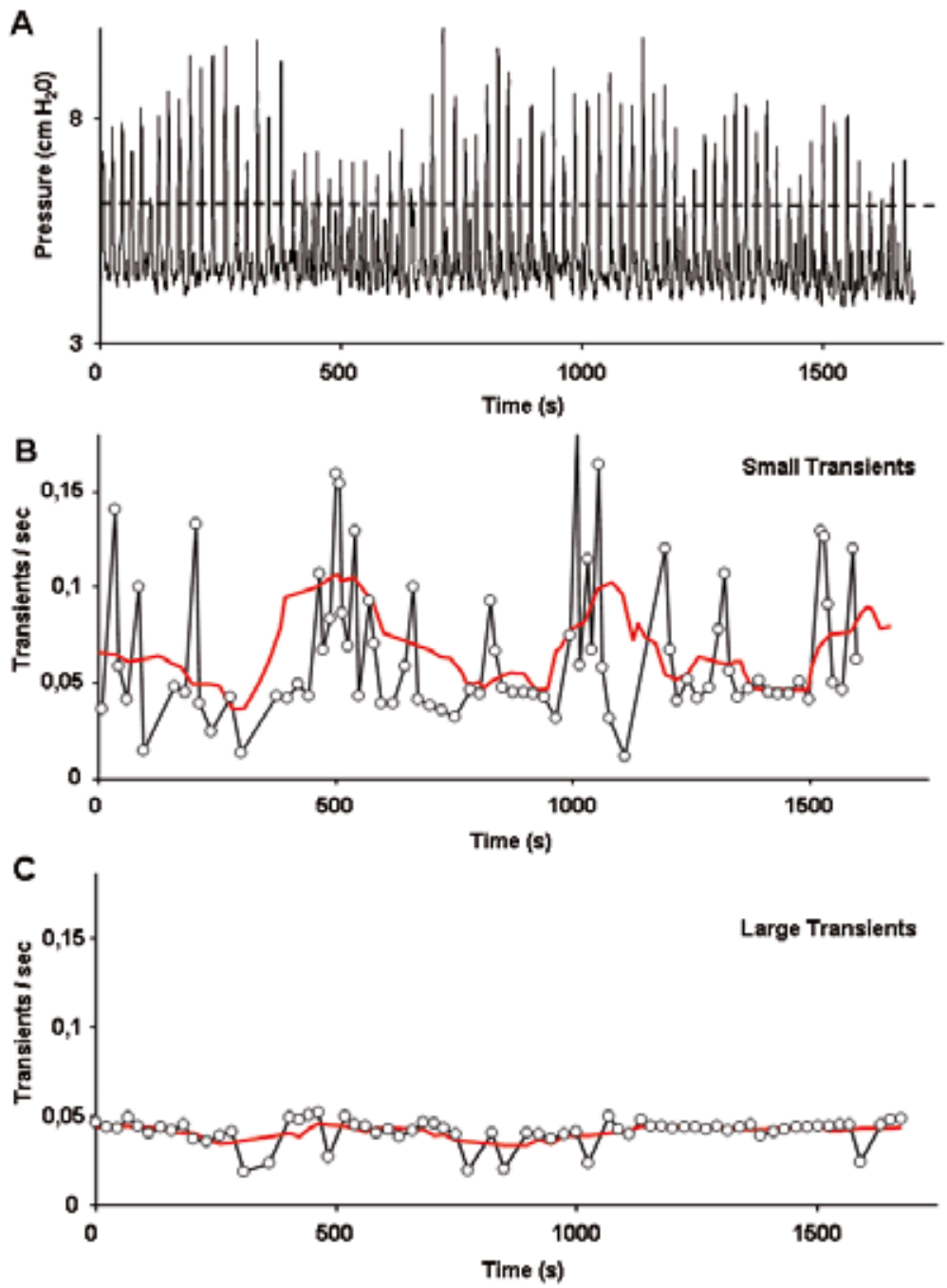

Figure 6. A, part of a medium volume isovolumetric trace. B and C, instantaneous frequency split into small and large amplitude NVCs respectively. Dashed line in A displays the median, which is the cut off point between small and large contractions (median amplitude $\mathrm{I}, 9 \mathrm{~cm} \mathrm{H}_{2} 0$ ). In accordance with the filling experiments the small NVCs display a phasic pattern in instantaneous frequency. Red line displays the moving average. C, with large NVC no phasic pattern or gradual increase in frequency is visible. Red line is the moving average. 
Structural and Functional Aspects of Sensory-motor Interaction in the Urinary Bladder

\section{Discussion}

The current study demonstrates the presence of NVCs during the filling phase in the anaesthetized guinea pig. In general, the NVCs progressively increase in amplitude and frequency as the bladder fills. In the awake rat different phases in non voiding activity have been described ${ }^{10}$. In the guinea pig no phases could be identified or separated, except a phase without NVCs and a phase with NVCs. Furthermore, in the rat the frequency of NVCs decreases just before micturition, which is not observed in the guinea pig. It is not clear whether these different observations are due to difference in species or use of anaesthesia. Ex vivo, the isolated whole guinea pig bladder also shows NVCs, which are small $\left(0,2-3 \mathrm{~cm} \mathrm{H}_{2} 0\right)$ and regular, and increase in frequency and amplitude as bladder volume increases ${ }^{3}$.Since the bladder is disconnected from the central nervous system, the generation and modulation of the NVCs must be an intrinsic property of the bladder wall. The current in vivo isovolumetric data show a phasic pattern in the frequency of NVCs. Small amplitude NVCs seem to be the source of these bursts of increased frequency (Figure 6). This phasic pattern in frequency is also seen with gradual filling of the bladder (Figure 3).Again, small amplitude NVCs are the source of this phasic pattern. Such a pattern is absent in the isolated whole bladder ${ }^{3}$.This suggests that it is evoked by spinal reflexes and might play a role in 'sensing' bladder volume. In the isovolumetric data the frequency of large amplitude NVCs is stable and does not increase, whereas in the filling experiments the frequency of large NVCs gradually increases with increasing volume. Previous afferent recording studies have shown that NVCs may elicit phasic firing of bladder afferents, suggesting that this form of bladder activity might underlie some form of motor-generated sensory function ${ }^{11,14-17}$. Both ex vivo and in vivo analysis of bladder filling at physiological rates revealed at least two different types of afferent activity ${ }^{16-18}$. One group showed phasic firing, which mirrored spontaneous contractions, while the other showed a continuous increasing firing rate during filling as a function of stretch ${ }^{16-18}$. Filling at a higher physiological rate shows similar afferent firing patterns ${ }^{16}$. However, at supra-physiological filling rate all afferents displayed the same pattern of continuous increased firing as the bladder fills.As filling rate increased the sensitivity of afferents toward bladder filling decreased ${ }^{18,19}$.

In the guinea pig, using various stimuli several functionally distinct populations of afferents have been identified, activated by different mechanisms ${ }^{20}$.These can be stretch-sensitive (muscle mechanoreceptors and tension-mucosal mechanoreceptor) or stretch-insensitive (mucosal mechanoreceptors and chemoreceptors) ${ }^{19,21}$. In vivo, two types of stretch-sensitive afferents were reported, one with firing linearly related to intravesical pressure, while the second type, firing in a phasic pattern, reached a plateau or even decreased at higher pressure ${ }^{16}$. This last type of afferent has been suggested to act like "volume receptors", sensing bladder distension ${ }^{22}$. This type of afferent fiber could be involved in the generation of small NVCs, underlying the phasic pattern as described in this study.

In conclusion, this study shows that NVC are present in the guinea pig bladder in vivo, and that small amplitude NVCs exhibit a specific frequency pattern. The exact role of these NVCs is currently unknown, but they may be related to sensory function as their pattern is only present when the bladder is connected to the spinal cord. How this correlates to humans is still unclear, as no method is yet available to accurately measure small bladder pressure increases in humans. 


\section{References}

I. van Waalwijk van Doorn, E. S., Remmers, A., Janknegt, R. A.: Conventional and extramural ambulatory urodynamic testing of the lower urinary tract in female volunteers. J Urol, 147: 1319, 1992

2. Robertson, A. S.: Behaviour of the human bladder during natural filling: the Newcastle experience of ambulatory monitoring and conventional artificial filling cystometry. Scand J Urol Nephrol Suppl, 20I: 19, 1999

3. Drake, M. J., Harvey, I. J., Gillespie, J. I.: Autonomous activity in the isolated guinea pig bladder. Exp Physiol, 88: 19, 2003

4. Drake, M. J., Hedlund, P., Harvey, I. J. et al.: Partial outlet obstruction enhances modular autonomous activity in the isolated rat bladder. J Urol, 170: 276, 2003

5. Gillespie, J. I., Harvey, I. J., Drake, M. J.: Agonist- and nerve-induced phasic activity in the isolated whole bladder of the guinea pig: evidence for two types of bladder activity. Exp Physiol, 88: 343, 2003

6. Gillespie, J. I.: The autonomous bladder: a view of the origin of bladder overactivity and sensory urge. BJU Int, 93: 478, 2004

7. Gillespie, J. I.: Noradrenaline inhibits autonomous activity in the isolated guinea pig bladder. BJU Int, 93 : 40I, 2004

8. Gillespie, J. I.: Modulation of autonomous contractile activity in the isolated whole bladder of the guinea pig. BJU Int, 93: 393, 2004

9. Finney, S. M., Stewart, L. H., Gillespie, J. I.: Volume-induced responses in the isolated bladder: evidence for excitatory and inhibitory elements. BJU Int, 102: II54, 2008

10. Streng, T., Hedlund, P., Talo, A. et al.: Phasic non-micturition contractions in the bladder of the anaesthetized and awake rat. BJU Int, 97: 1094, 2006

II. Coolsaet, B. L., Van Duyl, W. A., Van Os-Bossagh, P. et al.: New concepts in relation to urge and detrusor activity. Neurourol Urodyn, 12: 463, 1993

12. Davies, B., Morris, T.: Physiological parameters in laboratory animals and humans. Pharm Res, 10: 1093 , 1993

13. Watt, J. A., Dickinson, R. G.: The effect of diethyl ether, pentobarbitone and urethane anaesthesia on diflunisal conjugation and disposition in rats. Xenobiotica, 20: 289, 1990

14. McCarthy, C. J., Zabbarova, I. V., Brumovsky, P. R. et al.: Spontaneous contractions evoke afferent nerve firing in mouse bladders with detrusor overactivity. J Urol, I8I: I459, 2009

15. Habler, H. J., Janig, W., Koltzenburg, M.: Myelinated primary afferents of the sacral spinal cord responding to slow filling and distension of the cat urinary bladder. J Physiol, 463: 449, 1993

16. Shea, V. K., Cai, R., Crepps, B. et al.: Sensory fibers of the pelvic nerve innervating the Rat's urinary bladder. J Neurophysiol, 84: 1924, 2000

17. Yu, Y., de Groat, W. C.: Sensitization of pelvic afferent nerves in the in vitro rat urinary bladder-pelvic nerve preparation by purinergic agonists and cyclophosphamide pretreatment. Am J Physiol Renal Physiol, 294: FII46, 2008

18. De Wachter, S., De Laet, K., Wyndaele, J. J.: Does the cystometric filling rate affect the afferent bladder response pattern? A study on single fibre pelvic nerve afferents in the rat urinary bladder. Neurourol Urodyn, 25: 162, 2006 
Structural and Functional Aspects of Sensory-motor Interaction in the Urinary Bladder

19. Moss, N. G., Harrington, W. W., Tucker, M. S.: Pressure, volume, and chemosensitivity in afferent innervation of urinary bladder in rats. Am J Physiol, 272: R695, 1997

20. Zagorodnyuk, V. P., Gibbins, I. L., Costa, M. et al.: Properties of the major classes of mechanoreceptors in the guinea pig bladder. J Physiol, 585: 147, 2007

21. Zagorodnyuk, V. P., Costa, M., Brookes, S. J.: Major classes of sensory neurons to the urinary bladder. Auton Neurosci, 126-127: 390, 2006

22. Morrison, J.: The activation of bladder wall afferent nerves. Exp Physiol, 84: I3I, 1999 


Chapter 5

\section{Changes in Bladder Innervation in a Mouse Model of Alzheimer's Disease}

Bart T. Biallosterski, Stefan G.G. de Wachter, Gommert A. van Koeveringe, Philip E.V. Van

Kerrebroeck, Jan de Vente, Monique T. Mulder and James I. Gillespie

PMID: 20025962

Journal of Chemical Neuroanatomy 2010 
Structural and Functional Aspects of Sensory-motor Interaction in the Urinary Bladder

\begin{abstract}
Purpose

The aims of this study were to compare the structure of bladders from a transgenic mouse model of Alzheimer's disease with age matched control animals and to explore the idea that any structural differences might be related to functional bladder changes associated with the condition.
\end{abstract}

\title{
Materials and Methods
}

Two groups of mice were used. Transgenic animals in which the murine Amyloid Precursor Protein (APP) gene has been partly replaced by the human APP including both the Swedish and London mutations and that overexpress a mutant of the human Presenilin I gene (PSIMI46L) driven by the PDGF promoter. The transgenic mice (APP ${ }^{\text {SL }} /$ PSI ${ }^{\text {MI } 46 L}$ ) aged $24+3$ months were used. The second group was an age matched control group of C57 black mice. The bladders from each group were isolated, fixed in $4 \%$ paraformaldehyde and prepared for immunohistochemistry. Antibodies to the vesicular acetylcholine transporter (VAChT) and neuronal nitric oxide synthase (nNOS) were used to identify neural structures.

\section{Results}

Cholinergic nerves $\left(\mathrm{VAChT}^{+}\right)$were observed in the inner and outer muscle bundles of APP ${ }^{\text {SL/PSIMI46L }}$ and control mice. No major differences were noted in the distribution of these fibres. In contrast, there was a distinct difference in the innervation of the sub-urothelial layer. In App I ${ }^{\text {SL/PSIMI46L }}$ mice there were numerous VAChT and nNOS positive fibres in sharp contrast to the paucity of similar nerves in control animals. VAChT and nNOS did not appear to co-localise in the same nerve fibres within the lamina propria. Pairs of nerve fibres, $\mathrm{nNOS}^{+}$and $\mathrm{VAChT}^{+}$, were observed to be intertwined and run in close proximity. A particularly unusual feature of the APP ${ }^{S L} /$ PSI $^{\text {MI }}{ }^{\text {LL }}$ mouse bladder was the presence of neurones within the bladder wall. These nerve cell bodies were seen in all ApP ${ }^{S L} / P S I^{M / 46 L}$ mouse bladders. The neurones could be found singly or in small ganglion like groups of cells and were located in all layers of the bladder wall (sub-urothelium, in the lamina propria adjacent to the inner muscle and within the inner muscle and outer muscle layers). No nerve cells or small ganglia were noted in any of the control bladders studied.

\section{Conclusions}

There are structural differences in the bladders of APP ${ }^{S L} / P S I^{M I 46 L}$ mice compared to control animals. These differences are associated with sub-urothelial nerves which, because of their location, are likely to be sensory fibres. This may lead to a changed sensory processing from the APP ${ }^{\text {SL/PSIMI46L }}$ bladders. The physiological role of the intra-mural neurones and ganglia is not known. It is speculated that they may be associated with peripheral motor/sensory mechanisms linked to the generation and modulation of sensation. 


\section{Introduction}

Dementias, including Alzheimer's disease, are disabling conditions that can severely affect the quality of life for those who suffer. For many such patients incontinence and more so urgency are major additional problems which seriously affects on their care and lifestyle 1,2. It is generally accepted that dementia related incontinence is linked to cognitive impairment and reduced awareness. Less emphasis has been placed on possible changes in the lower urinary tract of such patients that might contribute to and exacerbate the problem. Experimental investigation of this problem in patients with dementia is difficult from moral and ethical grounds. Transgenic animals have provided an important approach to understanding the changes in dementias. Animals have been bred in which specific mutations have been engineered which mimic, in part, the changes seen in dementias including Alzheimer's disease. One such model involves a double mutation: One mutation is to the Amyloid Precursor Protein (APP) which includes both the Swedish and London mutations (KM6706/67INL and V7I7I, ${ }^{3}$ ) and the second to the PSIMI46L mutation (PSI MI46L, ${ }^{4}$ ). These double transgenic mice (APP ${ }^{\text {SL/PSIMI46L) }}$ ) exhibit numerous changes in their central nervous system ${ }^{3-6}$. Including increased amyloid deposits leading to plaque formation, with enlarged cholinergic terminals associated with and confined to the periphery of compact plaques. The model also shows activated astrocytes and microglia clusters. Since many of these changes are seen in patients, post mortem, with Alzheimer's disease the model is considered an appropriate one with which to study the cellular and molecular elements of Alzheimer's disease and its consequences ${ }^{7}$.

In this study we have used this double transgenic mouse model to explore the idea that there are peripheral changes in the bladder of these animals that might contribute to the overall pathophysiological changes that occur. The intention was to explore the idea that peripheral changes in the lower urinary tract might contribute to or exacerbate lower urinary tract dysfunction in patients with dementia. This study focuses on the distribution of nerve fibres in the bladder wall using nitric oxide synthase ( $\mathrm{nNOS}$ ) which is widely distributed in the lower urinary tract and may be important as an inhibitory neurotransmitter released by afferent nerves ${ }^{8,9}$. Vesicular acetylcholine transporter (VAChT) is used as a marker of cholinergic nerves. As there is an intimate relationship between the cholinergic innervation and nitric oxide synthase in the bladder, we investigated the effect of $\mathrm{NO}$ signalling in cholinergic fibres ${ }^{10}$. We were interested in studying the distribution of cholinergic fibres in normal mice and in a mice-model for AD.

\section{Materials and Methods}

All experimental procedures were approved by the local ethical committee of the Maastricht University for animal experiments according to the Netherlands' governmental guidelines. Mice with gene-targeted expression of the APP mutant encoding the Swedish/London-FAD mutations (APPSL) were generated as described by Köhler et al. ${ }^{3}$. The APPSL mice were crossbred with PSI(MI46L) transgenic mice ${ }^{4}$ and back crossed on a C57B|6/J background. 
Structural and Functional Aspects of Sensory-motor Interaction in the Urinary Bladder

Two groups of ApP ${ }^{\text {SL }} / P S I^{\text {MI46L }}$ mice, male or female, were used; C57BI6/J control mice aged 21 to 28 month old $(n=6)$ and a double transgenic mouse model of Alzheimer's disease $(n=10)$ aged 24 months to 26 months old. The double transgenic mice were developed at the department of Psychiatry and Neuropsychology at Maastricht University. Genetic screening was performed to ensure that a copy of each of the aforementioned mutations was present. Two separate single PCR biological assays were carried out using a thermal cycler (BIO-RAD thermal cycler, Bio-Rad Laboratories, Hercules, CA, USA). Primers were chosen for the specific mutations. The animals were housed in a temperature and light (I2h light/dark cycle) controlled room and allowed free access to food and water. The mice were killed by cervical dislocation. The urinary bladder was removed and placed in ice-cold Krebs solution containing $121.1 \mathrm{mM} \mathrm{NaCl}, 1.87 \mathrm{mM} \mathrm{KCl}$, $1.2 \mathrm{mM} \mathrm{CaCl}_{2}, \mathrm{I} .15 \mathrm{mM} \mathrm{MgSO}_{4}, 25 \mathrm{mM} \mathrm{NaHCO}_{3}$, I.I7 mM KH $\mathrm{PO}_{4}, 11.0 \mathrm{mM}$ glucose, bubbled with $5 \% \mathrm{CO}_{2}$ and $95 \% \mathrm{O}_{2}(\mathrm{pH} 7.4)$. Each bladder was divided in two pieces and fixed in an ice-cold solution of $4 \%$ freshly prepared depolymerised paraformaldehyde in 0.I M phosphate buffer $(\mathrm{pH} 7.4)$ for $120 \mathrm{~min}$ at $4^{\circ} \mathrm{C}$. After this the tissues were washed at $4{ }^{\circ} \mathrm{C}$ in 0. IM phosphate buffer containing $10 \%$ sucrose $(24 \mathrm{~h}), 20 \%$ sucrose $(24 \mathrm{~h})$ and $30 \%$ sucrose $(24 \mathrm{~h})$. The tissues from the lateral wall were snap-frozen with isopentane cooled in liquid nitrogen placed in Tissue-Tek O.C.T. compound (Bayer Corporation, Pittsburgh, PA, USA), or frozen into O.C.T. compound using carbon dioxide, to form a single block. Cryostat sections $(10 \mu \mathrm{m})$ were cut in a manner so that each section was perpendicular to the urothelial surface. Sections were then thawed onto chrome-alum-gelatincoated slides and processed for immunohistochemistry.

\section{Immunohistochemistry}

Sections were dried for $60 \mathrm{~min}$ at room temperature followed by three washes with Tris-buffered saline (TBS; $\mathrm{pH}$ 7.6) for $5 \mathrm{~min}$. and thereafter incubated overnight with primary antibodies at $4{ }^{\circ} \mathrm{C}$. Primary antibodies were diluted in TBS containing $0.3 \%(\mathrm{v} / \mathrm{v})$ Triton X-100 (TBS-T). The rabbit antibody against Vesicular Acetylcholine Transporter (VAChT, dilution I:I000, SigmaAldrich) was used to visualize cholinergic fibres. Neuronal nitric oxide synthase was visualized using a sheep anti-nNOS antibody (nNOS, dilution I:1000, generous gift from Dr. P.C. Emson). After overnight incubation with the primary antibodies, sections were washed in TBS-T, TBS and TBS-T; each wash step lasted 15 min. Rabbit primary antibody was visualized using Alexa Fluor 488 donkey anti-rabbit IgG $(\mathrm{H}+\mathrm{L})$ conjugate (Molecular Probes), diluted I: 100 in TBS-T. Sheep primary antibody was visualized with Alexa Fluor 594 donkey anti-sheep IgG conjugate (Molecular Probes), diluted I:I00. Sections were incubated with the secondary antibodies for $90 \mathrm{~min}$ at RT in the dark. After washing, the sections were mounted with TBS-glycerol (80\%). Typically for each bladder the staining was done in duplicate and repeated at least on 2 separate days. Only the lateral wall was studied and all observations were accumulated from the different slides and from different bladders. 
Sections were photographed using an Olympus AX70 microscope using $\times 10, \times 20$ and $\times 40$ (oil) objectives. For the detection of Alexa 488 fluorescence we used a narrow band-pass MNIBA-filter and for the detection of Alexa 594 we used a filter with a narrow excitation band the U-M4I007A filter ((525 nm - $560 \mathrm{~nm})$, both filters are from Chroma Technologies, Rockingham, Vt, USA). The microscope was equipped with a cooled CCD Olympus Digital video camera F-view. Images were stored digitally as 16 bit images by using the computer program cell- $\mathrm{P}^{\circledR}$ (Olympus, Germany). The number of grey values was reduced using a linear function to 4095 . We also examined the slides with confocal microscope technique, several photomicrographs in the article are acquired with this microscope, using a Bio-Rad MRC600 confocal microscope (Bio-Rad Laboratories Ltd, Hemel Hempstead, UK) equipped with an air-cooled Argon- Krypton mixed gas laser and mounted onto an Axiophotemicroscope (Zeiss), using oil-immersion objectives (40X, NA 1/4 3DI.3 or 63X, NA1/4 3DI.4). The laser-scanning microscope was used in dual wavelength excitation at 488 and $568 \mathrm{~nm}$. Optical sections were recorded in the Kallman filtering mode using 4-8 scans for each picture. Z-series were generated by collecting a stack consisting of optical sections using a step size between 0.18 and $0.46 \mathrm{~mm}$ in the $z$-direction. Images were arranged with the program Microsoft Powerpoint 2003 (Microsoft Corporation, Seattle, WA, USA).

\section{Results}

Figure I shows photomicrographs of sections from the bladder wall from a control mouse (A) and an APP ${ }^{S L} / P S I^{M I 46 L}$ mouse (B). The sections were stained using an antibody to vesicular acetylcholine transporter (VAChT) in order to identify cholinergic nerve fibres. A dense cholinergic innervation is seen in the muscle layers of control and APP ${ }^{S L} / P S I^{M I 46 L}$ bladders $(m)$. No major differences in the inner muscle layer were noted between control and APP ${ }^{\mathrm{SL}} / \mathrm{PSI} \mathrm{II}^{\mathrm{M} / 6 \mathrm{~L}}$ bladders, although positive neurons were observed in the lamina propria adjacent to the inner muscle layer (Figure IB).

In contrast, there was a clear difference in the number of nerve profiles in the lamina propria in the $A_{P P}$ SL/PSI ${ }^{M I 46 L}$ compared to controls. In the control bladders few positive nerves were found while in the APP ${ }^{S L} / P S I^{M I 46 L}$ bladders there was a high density of sub-urothelial nerves expressing VAChT or $\mathrm{nNOS}$ (\# Figure IA and B). In addition, the APP ${ }^{S L} / P S I^{M I 46 L}$ bladders had single nerve cells (* Figure IB) which were not found in any of the control animals. These differences are shown in greater detail in the subsequent figures. 

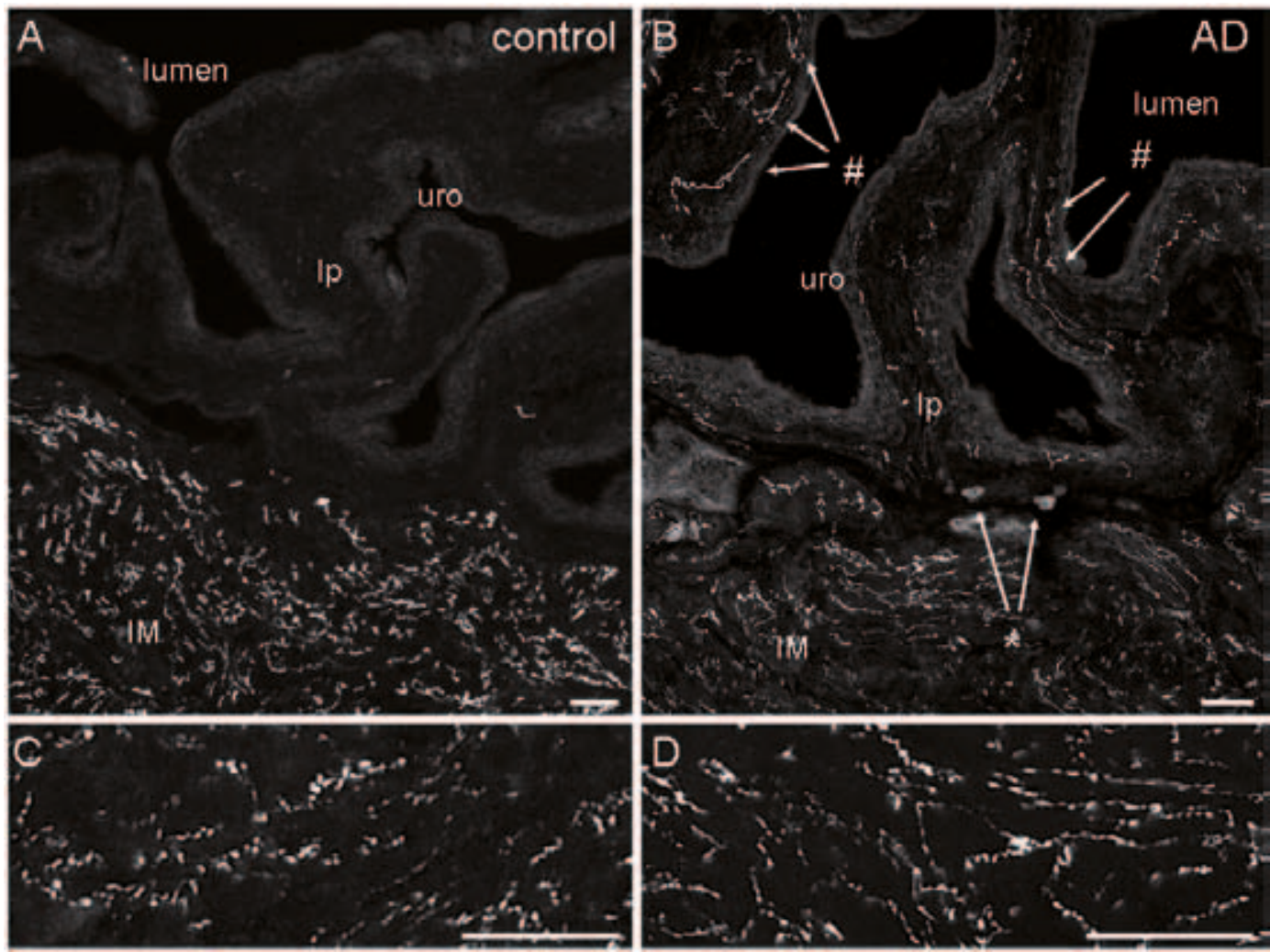

Figure I. Photomicrographs of vesicular acetylcholine transporter (VAChT) associated with nerve fibres in the lamina propria and inner muscle layer of the bladders of 24 month old C 57 black mice (A: control) and the double transgenic model $A_{\text {AP }}{ }^{\text {SL }}$ PSI II46L (B: Alzheimer's disease model). In each panel the urothelium (uro), lamina propria (IP) and inner muscle (IM) layers are shown. NB the absence of structures staining with $\mathrm{VAChT}$ associated with the urothelium and sub-urothelium in the control mice. This is in contrast to the presence of numerous structures in the APP ${ }^{\text {SL/PSI }}{ }^{\text {I146L }}$ mice (\#). In panel B

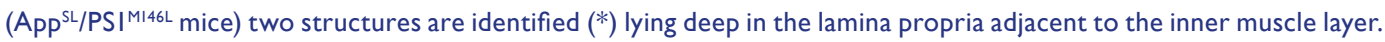
These are single nerve cell bodies as described in detail in Figures 4 and 5. Panels $C$ and $D$ show the uniform distribution of VAChT in the muscle layers of both mice. Calibration bars in all panels are $30 \mathrm{~mm}$. 

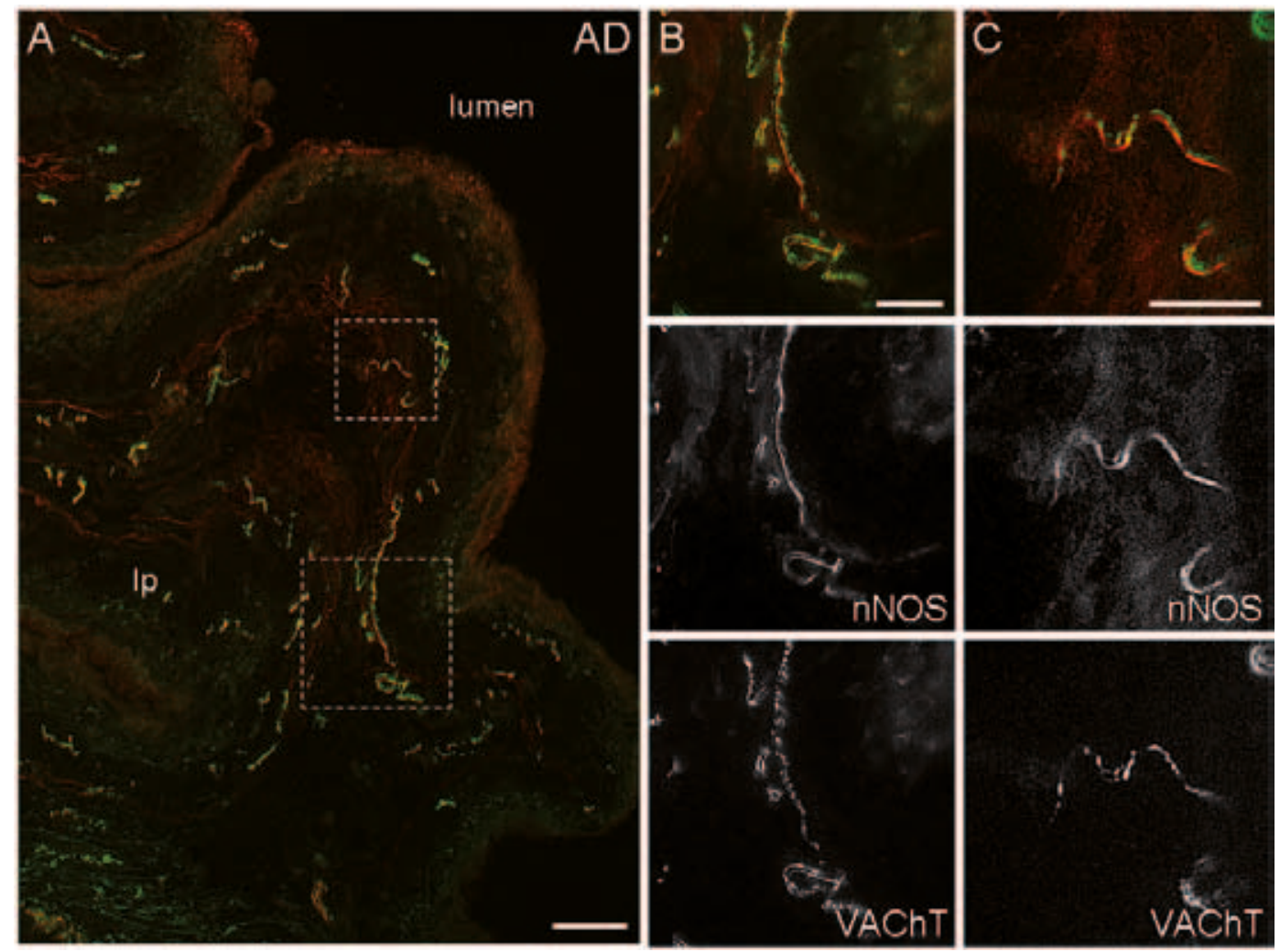

Figure 2. Photomicrographs of nerve fibres in the lamina propria of ApP ${ }^{S L} / P S I^{M I 46 L}$ mice (Ip). The section was stained with antibodies to VAChT (green) and nNOS (red). Panel A; image at low magnification demonstrating the density of innervation of this region of the wall. Panels $B$ and $C$ are regions in panel $A$ delineated by the dashed squares at higher magnification. VAChT and $\mathrm{nNOS}$ fibres are seen as distinct structures with little co-localisation indicative of two distinct populations of nerves in this region. Calibration bars: $20 \mathrm{~mm}$ in $\mathrm{A}$ and $10 \mathrm{~mm}$ in $\mathrm{B}$ and $\mathrm{C}$.

nNOS and VAChT fibres were demonstrated in the lamina propria of the APP ${ }^{\text {SL/PSIMI46L }}$ mice (Figure 2). Typically, small bundles containing 2-3 fibres are found but often single axons could be seen. The fibres appear to be at least two distinct types: $\mathrm{VAChT}^{+}$and $\mathrm{nNOS}^{+}$fibres. No definitive fibres are noted which co-localised $\mathrm{VAChT}^{+}$and $\mathrm{nNOS}^{+}$. A common structural feature is the close apposition of two nerves, one $\mathrm{VAChT}^{+}$and the other $\mathrm{nNOS}^{+}$. These fibre pairs are seen to run in close proximity to each other as they traversed the lamina propria to innervate the urothelium. Since no efferent system has yet been described for the urothelium these $\mathrm{VAChT}^{+}$and nNOS ${ }^{+}$fibres and intertwined nerves could be afferent. As in previous studies on the mouse bladder no ganglia or nerve cell bodies were seen within the lamina propria or the muscle layers. 


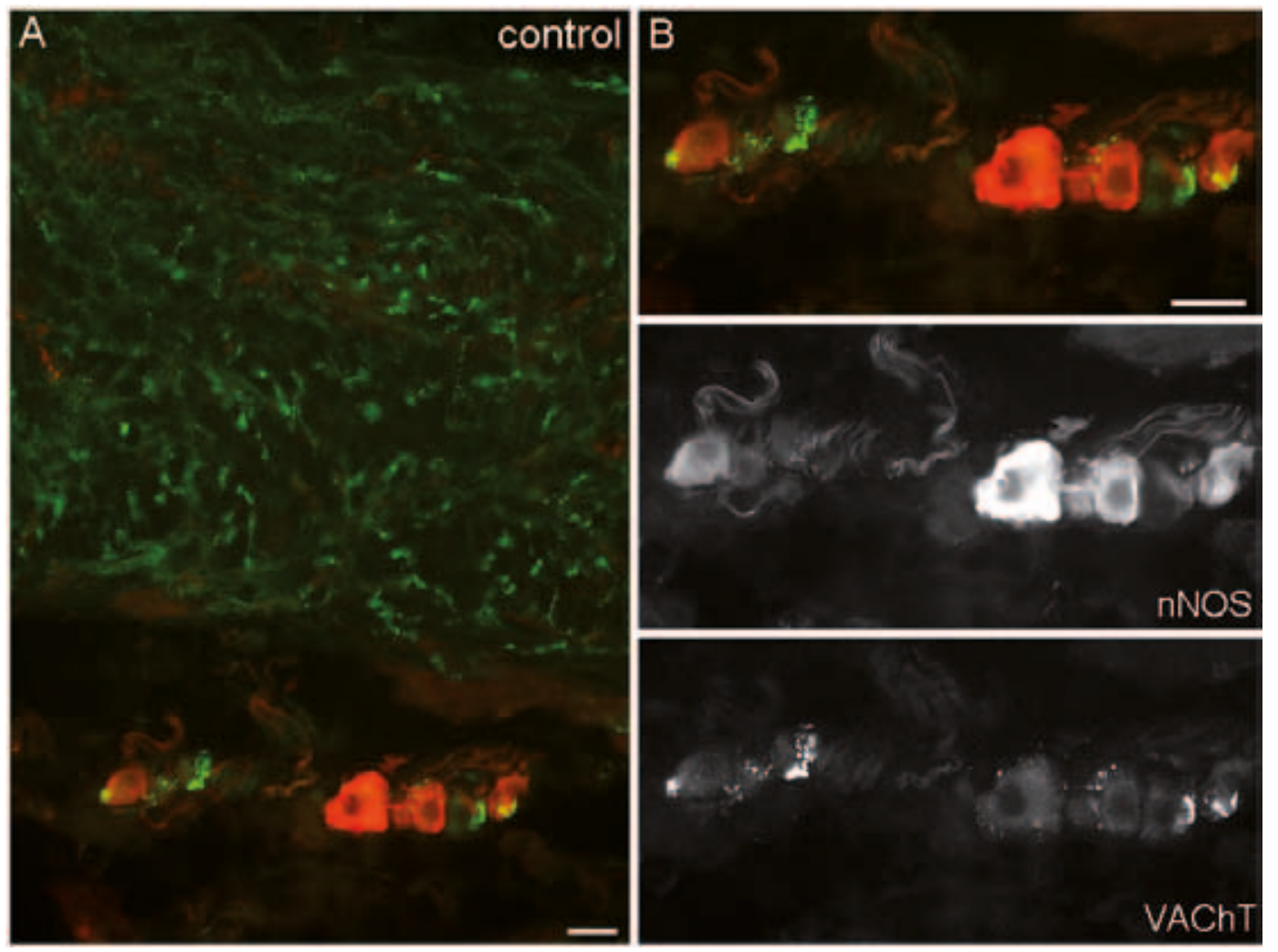

Figure 3. Photomicrograph of a small external ganglion associated with an aged control mouse. The section is stained for VAChT (green) and $\mathrm{nNOS}$ (red). The collection of nerve cell bodies is seen on the outer surface of the bladder wall. This region is shown at higher magnification in B where the individual images of the VAChT and $\mathrm{nNOS}$ stainings are illustrated. Calibration bars: $20 \mathrm{~mm}$ in A and $25 \mathrm{~mm}$ in B.

Small ganglia are observed on the outer surface of the bladder (Figure 3). These ganglia appear to consist of different types of neurones with some being strongly positive for $\mathrm{nNOS}$. Some nerve cell bodies are also associated with $\mathrm{VAChT}^{+}$structures that may be the terminals of cholinergic fibres (Figure 3B). 

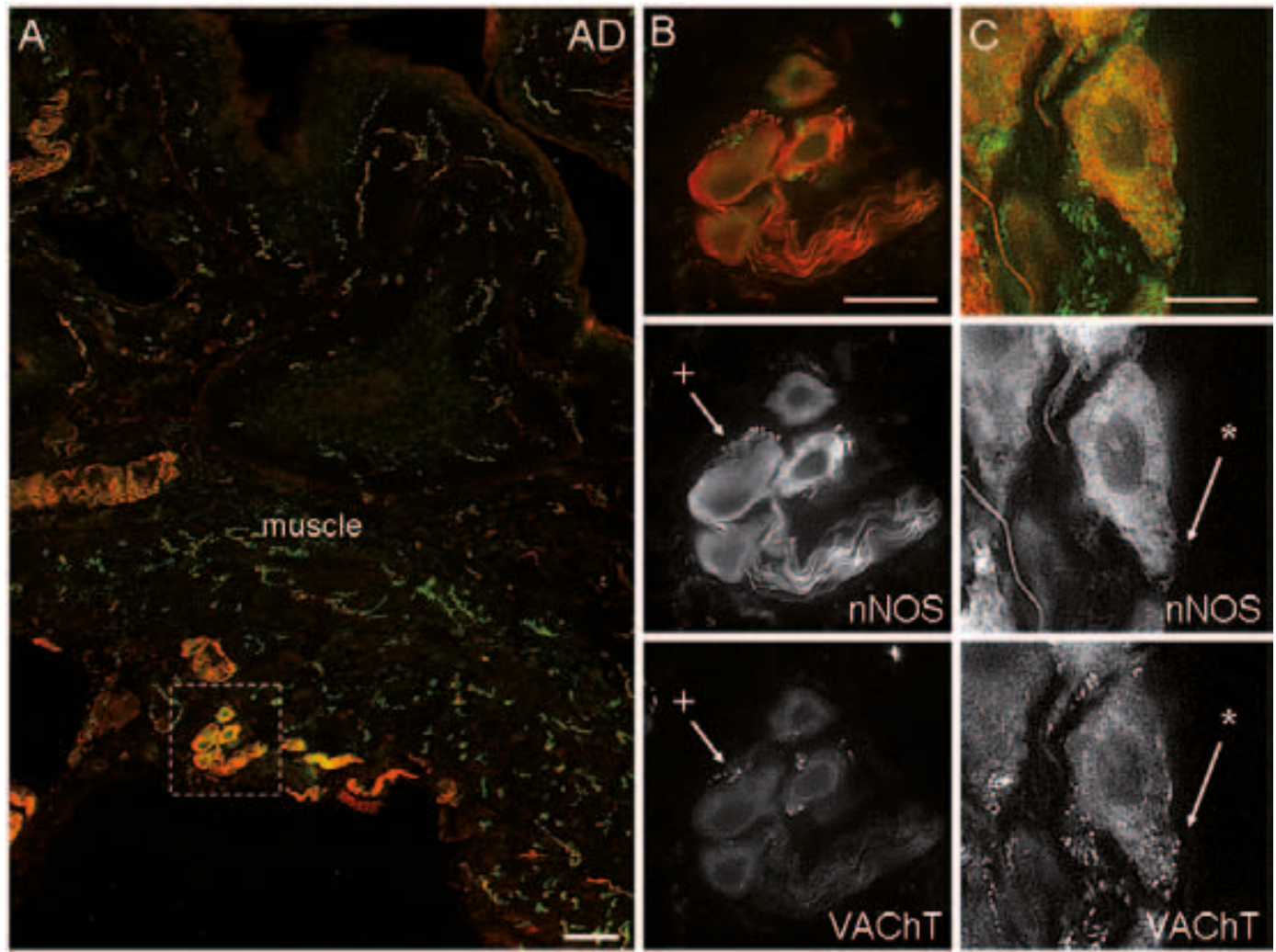

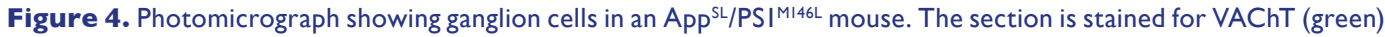
and $\mathrm{nNOS}$ (red). The region marked by the dashed square is shown at higher magnification in B. 4 nerve cell bodies are clearly seen with their nuclei. + Arrow points to a region on one of the cell bodies where punctuate staining for VAChT and $\mathrm{nNOS}$ can be seen. This staining may represent nerve terminal(s) on the cell body. $\mathrm{C}$ shows a nerve cell in an outer ganglion of a different APP ${ }^{S L} / P S I^{M 146 L}$ mouse. The ganglion displays co-localisation of VAChT and nNOS antibodies. The lower panel clearly shows the punctuate VAChT staining close to the nerve cell body $(*)$. The upper panel shows a nNOS positive nerve fibre with no VAChT staining present. Calibration bars: $30 \mathrm{~mm}$ in A, $20 \mathrm{~mm}$ in B and $15 \mathrm{~mm}$ in C.

Similar ganglia are observed on the outer surface of the bladder in the APP ${ }^{\text {SL }}$ PSI ${ }^{\text {MI } 46 L}$ mice (Figure 4). These ganglia are distinctive and typically existed as small groups of several nerve cell bodies. Discrete spots of VAChT fluorescence are often seen associated with neurones within these ganglia suggesting that they also might receive a cholinergic input (Figure $4 \mathrm{~B}+$ arrows and $C *$ arrows). In Figure 4C the ganglion displays distinct co-localisation of the VAChT and nNOS. 

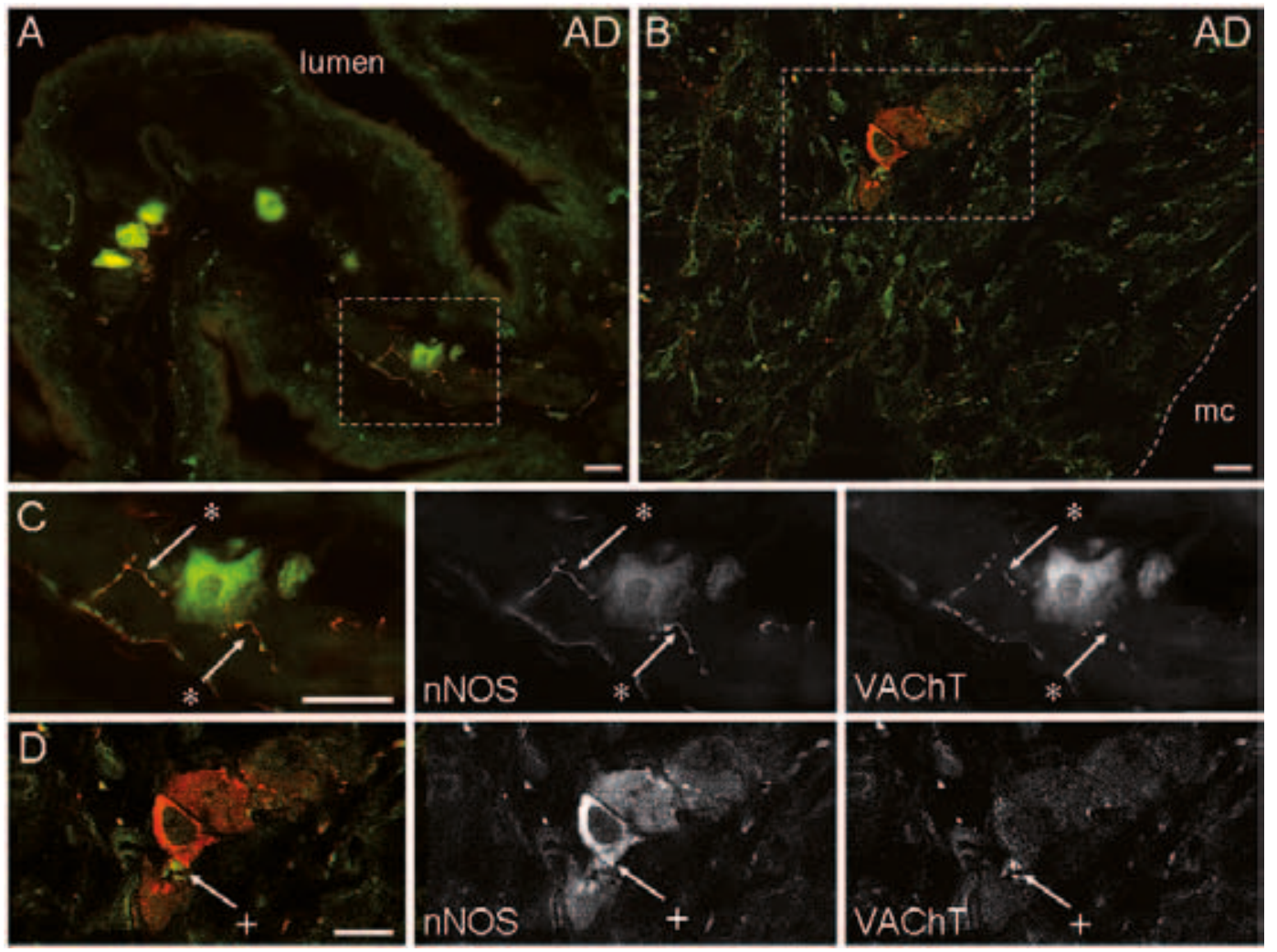

Figure 5. Examples of ganglion cells lying within the lamina propria and the muscle layer of the APP ${ }^{S L} / P S I^{M 146 L}$ mouse: A, close to the urothelium B, in the muscle. The sections are double stained for VAChT (red) and nNOS (green). C and D show magnified regions from $A$ and $B$ respectively. In $C$ nerve fibres are seen to come in close contact with the ganglion cells and in DVAChT-positive terminals are seen close to the ganglion cell body. The muscle coat is delineated by the dashed line and the abbreviation MC. Calibration bars: $20 \mathrm{~mm}$ in A, and B, C and D.

An unusual feature of the bladders from APP ${ }^{S L} / P S I^{M I 46 L}$ mice is the occurrence of nerve cell bodies in the bladder wall. Examples of these neurones are shown in Figures 5. In Figure 5 A and B examples are shown of cells in the in the lamina propria immediately below the urothelium and in the muscle respectively. Regions of these images are shown at higher magnification in panels $C$ and $D$. In both $\mathrm{C}$ and $\mathrm{D}$ nNOS ${ }^{+}$nerve cell bodies are seen in close proximity to $\mathrm{VAChT}^{+}$fibres and terminals. In the example shown in panel B, 3 neuronal cell bodies are clearly seen in close proximity. Close examination of this group at higher magnification and inspection of the individual images (panel D) shows that only two cell bodies are $\mathrm{nNOS}^{+}$suggesting that this population of intra-mural neurones may be heterogeneous. 

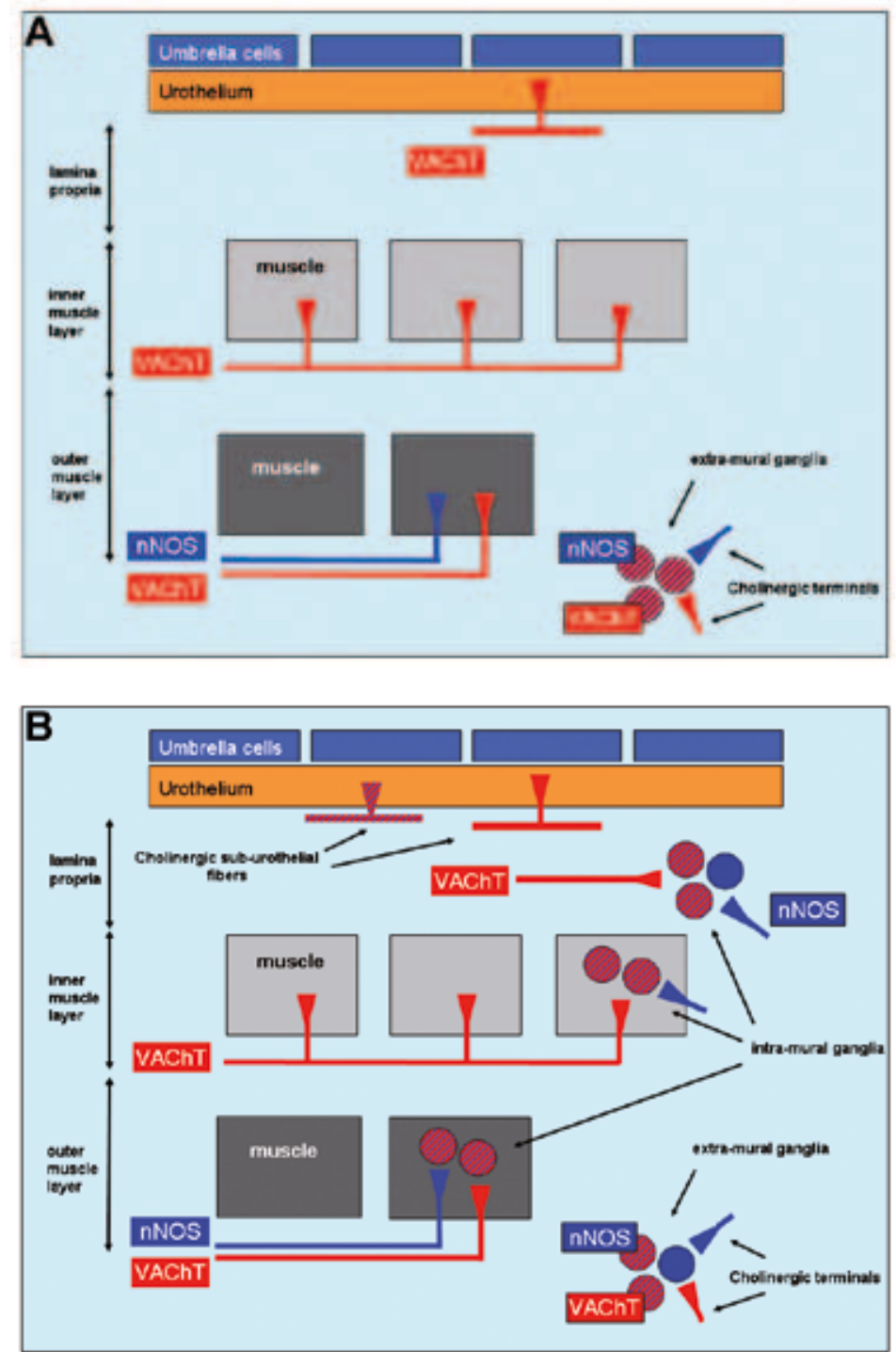

Figure 6. Cartoon consisting of two panels illustrating the major observations described in the text. Panel A displays the agematched control mice and panel B the ApP ${ }^{S L} / P S I^{M I 46 L}$ mice. Describing from the top, the urothelium, down to the muscle coat one can see an increased density of nerve fibres in the lamina propria in the ApPL/PSI ${ }^{\text {MI } 46 L}$ mice, in this layer one can also see the presence of nerve cell bodies (ganglia) and the appearance of terminals on the ganglia. Further down in de muscle layers no clear difference in density of the cholinergic innervation is seen. Here again nerve cell bodies can be seen in the APP ${ }^{\text {SL/PS I }}{ }^{\text {I46L }}$ mice. In both the aged matched control mice and the APP ${ }^{\text {SL }} / \mathrm{PS}$ I $^{\mathrm{MI}}{ }^{46 \mathrm{~L}}$ mice nerve cell bodies (ganglia) are found just of the bladder at the muscle coat.With most of these ganglia, nerve fibres possibly emanating terminals can be seen in close proximity. 
Structural and Functional Aspects of Sensory-motor Interaction in the Urinary Bladder

\section{Discussion}

The present observations demonstrate structural differences in the bladder wall of the App ${ }^{S L /}$ PSI ${ }^{\text {MI } 46 L}$ Alzheimer's mice compared to age matched controls. The major findings are summarised diagrammatically in Figure 6.

There are three major observations in this study. Firstly, the cholinergic innervation of the smooth muscle layers in the bladder wall, as shown by the $\mathrm{VACHT}$-IR, is not distinctly different between the $\mathrm{APP}^{\mathrm{SL}} / \mathrm{PSI} \mathrm{II}^{\mathrm{MLL}}$ mice and age matched controls (figure I, panels $\mathrm{C}$ and $\mathrm{D}$ ). This suggests that the ability of the bladder to contract will not be impaired in the transgenic mice allowing them to maintain normal voiding contractions. Therefore, the primary changes may not be directly associated with the efferent control of the bladder. The second major observation is a clear increase in the number of $\mathrm{VAChT}^{+}$and $\mathrm{nNOS}^{+}$nerve fibres within the lamina propria. It is generally assumed that nerves in this region of the bladder wall are sensory fibres contributing to the afferent output of the bladder. If this is so, the presence of $\mathrm{VAChT}^{+}$and $\mathrm{NOS}^{+}$fibres in the ApP ${ }^{S L} / \mathrm{PSI}{ }^{\text {MI }}{ }^{6 L}$ mice suggest alterations in the afferent system. The physiological consequences of such changes are not known but a higher density of afferent nerves might result in a different processing in or different sensory outflow from the bladder.

There are several different types of afferent fibres emanating from the bladder ${ }^{11-14}$. Some of these fibres are activated during normal bladder filling, whereas others may be activated by 'noxious' stimuli and may contribute to detrusor overactivity and overactive bladder symptoms ${ }^{15}$. It is still unclear which fibres only contribute to spinal reflexes and which give rise to conscious perception ${ }^{16}$. The altered expression of the VAChT and nNOS fibres in the lamina propria raises the interest to study the distribution of other nerve fiber types. For example Calcitonin Gene Related Peptide (CGRP) and transient potential receptor vanilloid I (TRPVI) fibers would be good candidates to study because they have been implicated in the occurrence of overactive bladder symptoms and Alzheimer patients have an increased prevalence of overactive bladder symptoms ${ }^{2,17-19}$. However, from immunohistochemistry studies we cannot say anything about the function of the putative afferent fibres in the APP ${ }^{S L} / P_{S} I^{M / 46 L}$ mice.

The afferent fibres, contributing to the perception of bladder volume, are a sub-set of sensory fibres $^{16}$. A similar heterogeneity in the nerves within the lamina propria and specifically associated with the urothelium has been reported in the normal guinea pig bladder ${ }^{20}$. As a third observation $\mathrm{VAChT}^{+}$fibres seen in the APP ${ }^{\mathrm{SL}} / \mathrm{PSI} \mathrm{II}^{\mathrm{M} 6 \mathrm{~L}}$ mice may be making contact with intramural ganglia (Figures 3 - 5). This anatomical arrangement has led to the suggestion that there may be local reflexes operating in the bladder wall ${ }^{20,21}$. Since there are no intra-mural ganglia in the normal mouse or rat bladder the concept of such local intra-mural reflexes in these species has not been considered ${ }^{22}$. There is, therefore, an important question regarding when and how the nerve cell bodies and ganglia appear in the ApP ${ }^{\mathrm{SL}} / \mathrm{PSI} \mathrm{MI}^{\mathrm{I} 6 \mathrm{~L}}$ mice.

We can conceive at least two possibilities. It may be that the genetic changes leading to the generation of the neurones occur at the embryonic stage and persist into adult life. There is no 
evidence for or against this at the present time but the data can and should be obtained. Secondly the nerves and ganglia may appear late in life along with the other changes seen in these animals associated with Alzheimer's disease. De novo generation of neurones is not a common phenomenon and therefore this explanation seems less likely and at this stage it is not plausible. Nerve cell bodies and small ganglia are found in the bladder in humans, pigs and guinea pigs ${ }^{20,23-25}$. Single nerve cell bodies are found but more often they are observed in small groups. The observation that they are associated with nerve fibres with terminal like structures has led to the suggestion that they receive synaptic inputs ${ }^{20,23,26}$. The presence of cholinergic terminals might imply that the intra-mural ganglia may receive both excitatory and inhibitory input where they may be a part of a motor/sensory system ${ }^{27,28}$. The properties and physiological role of these cells seen in the bladders of the APP ${ }^{S L} / P S I^{M I 46 L}$ mice are not yet clear. They may represent small intra-mural ganglia and perform a motor role similar to that proposed in the guinea pig ${ }^{20}$.

From this data we cannot infer anything about the origin or development of these differences nor can we say anything about their physiological consequences or extrapolate these findings to the human conditions of dementia or Alzheimer's. However, there are differences in the afferent and possibly the motor-sensory system in the mouse model, which may have some importance. These findings may aid in elucidating some of the underlying pathology for the lower urinary tract dysfunction in humans with dementia and Alzheimer's disease and illustrate the importance of the peripheral nervous. They may also help to stimulate research on changes in the peripheral nervous system in animals models initially designed to study disease of the central nervous system. Structural immunohistochemical studies of bladder tissue of patients with Alzheimer's disease and physiological studies on the present mouse model is a next step to take. 
Structural and Functional Aspects of Sensory-motor Interaction in the Urinary Bladder

\section{References}

I. Mattson, M. P.: Pathways towards and away from Alzheimer's disease. Nature, 430: 63I, 2004

2. Ransmayr, G. N., Holliger, S., Schletterer, K. et al.: Lower urinary tract symptoms in dementia with Lewy bodies, Parkinson disease, and Alzheimer disease. Neurology, 70: 299, 2008

3. Kohler, C., Ebert, U., Baumann, K. et al.: Alzheimer's disease-like neuropathology of gene-targeted APPSLxPSImut mice expressing the amyloid precursor protein at endogenous levels. Neurobiol Dis, 20: 528, 2005

4. Duff, K., Eckman, C., Zehr, C. et al.: Increased amyloid-beta42(43) in brains of mice expressing mutant presenilin I. Nature, 383: 710, 1996

5. Vanmierlo, T., Bloks, V. W., van Vark-van der Zee, L. C. et al.: Alterations in Brain Cholesterol Metabolism in the APPSLxPSI mut mouse, a Model for Alzheimer's Disease. J Alzheimers Dis, 2009

6. Vanmierlo, T., Rutten, K., Dederen, J. et al.: Liver $X$ receptor activation restores memory in aged AD mice without reducing amyloid. Neurobiol Aging, 2009

7. Rasmusson, D. X., Brandt, J., Steele, C. et al.: Accuracy of clinical diagnosis of Alzheimer disease and clinical features of patients with non-Alzheimer disease neuropathology. Alzheimer Dis Assoc Disord, 10: 180,1996

8. Fowler, C. J., Griffiths, D., de Groat, W. C.: The neural control of micturition. Nat Rev Neurosci, 9: 453, 2008

9. Hedlund, P.: Nitric oxide/cGMP-mediated effects in the outflow region of the lower urinary tract--is there a basis for pharmacological targeting of cGMP? World J Urol, 23: 362, 2005

10. de Vente, J., Markerink-van Ittersum, M., van Abeelen, J. et al.: NO-mediated cGMP synthesis in cholinergic neurons in the rat forebrain: effects of lesioning dopaminergic or serotonergic pathways on nNOS and cGMP synthesis. Eur J Neurosci, 12: 507, 2000

II. Shea, V. K., Cai, R., Crepps, B. et al.: Sensory fibers of the pelvic nerve innervating the Rat's urinary bladder. J Neurophysiol, 84: 1924, 2000

12. Morrison, J.: The activation of bladder wall afferent nerves. Exp Physiol, 84: I3I, 1999

13. Iggo, A.: Tension receptors in the stomach and the urinary bladder. J Physiol, 128: 593, 1955

14. De Laet, K., De Wachter, S., Wyndaele, J. J.: Systemic oxybutynin decreases afferent activity of the pelvic nerve of the rat: new insights into the working mechanism of antimuscarinics. Neurourol Urodyn, 25: 156, 2006

15. Wyndaele, J. J., De Wachter, S.: The sensory bladder (I): an update on the different sensations described in the lower urinary tract and the physiological mechanisms behind them. Neurourol Urodyn, 27: 274, 2008

16. Gillespie, J. I., van Koeveringe, G. A., de Wachter, S. G. et al.: On the origins of the sensory output from the bladder: the concept of afferent noise. BJU Int, 2009

17. Silva, C., Silva, J., Castro, H. et al.: Bladder sensory desensitization decreases urinary urgency. BMC Urol, 7: 9, 2007 
18. Liu, L., Mansfield, K. J., Kristiana, I. et al.: The molecular basis of urgency: regional difference of vanilloid receptor expression in the human urinary bladder. Neurourol Urodyn, 26: 433, 2007

19. Smet, P. J., Moore, K. H., Jonavicius, J.: Distribution and colocalization of calcitonin gene-related peptide, tachykinins, and vasoactive intestinal peptide in normal and idiopathic unstable human urinary bladder. Lab Invest, 77: 37, 1997

20. Gillespie, J. I., Markerink-van Ittersum, M., de Vente, J.: Sensory collaterals, intramural ganglia and motor nerves in the guinea-pig bladder: evidence for intramural neural circuits. Cell Tissue Res, 325: 33, 2006

21. Smet, P.J., Edyvane, K. A., Jonavicius, J. et al.: Neuropeptides and neurotransmitter-synthesizing enzymes in intrinsic neurons of the human urinary bladder. J Neurocytol, 25: II2, 1996

22. Gabella, G., Davis, C.: Distribution of afferent axons in the bladder of rats. J Neurocytol, 27: 14I, 1998

23. Smet, P. J., Jonavicius, J., Marshall, V. R. et al.: Distribution of nitric oxide synthase-immunoreactive nerves and identification of the cellular targets of nitric oxide in guinea-pig and human urinary bladder by cGMP immunohistochemistry. Neuroscience, 7I: 337, 1996

24. Dixon, J. S., Jen, P. Y., Gosling, J. A.: A double-label immunohistochemical study of intramural ganglia from the human male urinary bladder neck. J Anat, 190 (Pt I): 125, 1997

25. Pirker, M. E., Montedonico, S., Rolle, U. et al.: Regional differences in nitrergic neuronal density in the developing porcine urinary bladder. Pediatr Surg Int, 2I: I6I, 2005

26. Zhou, Y., Ling, E. A.: Colocalization of nitric oxide synthase and some neurotransmitters in the intramural ganglia of the guinea pig urinary bladder. J Comp Neurol, 394: 496, 1998

27. Gillespie, J. I.: The autonomous bladder: a view of the origin of bladder overactivity and sensory urge. BJU Int, 93: 478, 2004

28. Drake, M. J., Harvey, I. J., Gillespie, J. I.: Autonomous activity in the isolated guinea pig bladder. Exp Physiol, 88: 19, 2003 

Chapter 6

\section{Changes in Voiding Behaviour in a Mouse Model of Alzheimer's Disease}

Bart T. Biallosterski, Jos Prickaerts, Mohammad S. Rahnama'i, Stefan G.G. de Wachter, Gommert A. van Koeveringe and Celine Meriaux

Frontiers in Aging Neuroscience, 2015 
Structural and Functional Aspects of Sensory-motor Interaction in the Urinary Bladder

\begin{abstract}
Objectives

To determine whether the transgenic mouse model of Alzheimer's disease (AD), APPSL/PSIMI46L mouse, shows alteration of the urinary bladder function and anxiety, as for patients with $A D$, we examined the urinary marking behaviour in relation to affective behaviour.

\section{Materials and Methods}

At 18 months of age voiding behaviour of APPSL/PSIMI46L (APP/PSI) and wild type (WT) mice was assessed by using a modified filter paper assay in combination with video tracing, with the cage divided into a centre and corner zones. Anxiety-related behaviour and locomotion were respectively tested in an elevated zero maze and an open field.
\end{abstract}

\title{
Results
}

The APP/PSI mice urinated more in the centre zone than the WT mice. The total volume of markings was significantly lower in the APP/PSI mice. In both groups, the average volume of a marking in the corner zone was larger than in the centre zone. In the elevated zero maze task, the APP/PSI mice spent less time in the open arms of the arena than the WT mice. In the open field task, the APP/PSI mice covered a longer distance than the WT mice.

\section{Conclusions}

Our findings show that the APP/PSI mice have a different voiding behaviour compared to the WT mice, i.e. urinating with small volumes and voiding in the centre of the cage. We have seen increased locomotor activity and anxiety-related behaviours as factors in the change in voiding pattern, attributing to impairment of voiding functioning in the APP/PSI mouse. 


\section{Introduction}

In neurodegenerative disorders, like dementia and Alzheimer's disease (AD), patients can suffer from impairment of bladder function'. Incontinence often emerges when dementia has developed into a moderate disease stage $^{2}$. In general, people with sufficient cortical function commence voiding at an acceptable moment after going to a suitable location with the appropriate measures taken i.e. in a toilet and partially undressed. However, patients with dementia and AD especially can demonstrate uninhibited and unembarrassed voiding at an inappropriate place and/or time. This behaviour is often referred to as functional incontinence, and could be caused by dysfunctions at several levels of control. The general belief is that this incontinence is not derived from an abnormality in the lower urinary tract or its innervation, but from deficiencies in cholinergic neurotransmission in the cortical and subcortical areas of the central nervous system which then consequently leads to problems in locomotion and cognitive decline ${ }^{3}$. Cognitive severity and decline in dementia have been shown to significantly correlate to behavioural alterations, such as anxiety ${ }^{4}$. Several studies have linked anxiety to voiding dysfunction ${ }^{4,5}$. Many patients with AD also show signs of detrusor overactivity upon cystometric evaluation ${ }^{1,6}$. In the latter condition, dysfunctions in the integrated (neuro)-physiology of the bladder itself may contribute to this disabling condition. This is supported by a previous report from our group in which structural changes have been found in the bladder of a transgenic mouse model of $A D^{7}$. Consequently, all these factors could be responsible for the altered voiding behaviour seen in patients with AD. To gain insight into bladder function rodents are often studied using metabolic cages, allowing voiding frequency and volume to be measured while urine can be collected and analysed ${ }^{8,9}$. However, this method does not provide any information on the voiding behaviour of freely moving animals. Because AD is correlated with both anxiety and incontinencel, we wanted to investigate both the affective and the voiding behaviour of transgenic APPSL/PSI ${ }^{\text {MI } 46 L}$ (APP/PSI) mice, a mouse model of AD. We have previously used these mice, which have well-established cognitive impairment, clearly recognizable plaques and tangles and structural bladder changes, ${ }^{70}$. We hypothesize that this mouse model of AD displays an altered voiding behaviour compared to wild type (WT) mice. Voiding behaviour of APP/PSI and WT is compared by using a modified filter paper assay in combination with video-assisted tracing in time of moving and voiding patterns.

\section{Methods and Materials}

Animals were housed individually within a temperature-controlled environment with reversed I2hour light/I2-hour dark cycle (lights off from $7.00 \mathrm{~h}$ ) and standard chow and water available ad libitum at all times. All animals were subjected to one experiment per day for three consecutive days. The experiments were performed in the following order, (I) 'Voiding behaviour task' (VBT), (2) 'Elevated zero maze task' (EZM), and (3) 'Open Field task' (OF), respectively. This study was approved by the Animal Ethics Board of Maastricht University, The Netherlands. 


\section{Animals}

Mice with gene-targeted expression of the APP mutant encoding the Swedish/London-FAD mutations were generated as described previously" and crossbred with PSI ${ }^{\text {II46L }}$ transgenic mice to generate APPSL/PSI ${ }^{\mathrm{MI} 46 \mathrm{~L}}$ mice (APP/PSI). One-year-old wild type (WT) C57BL/6NCrl mice were purchased from Charles River Laboratories (L'Arbresle, France) and allowed to age in the same animal facility. At 18 months of age animals were divided into two groups, APP/PSI ( $n=7 ; 5$ females, 2 males) and WT ( $n=10$, males) mice were taken into experiment.

\section{Voiding behaviour task}

Mice were habituated to the test cage for approximately five hours, 24 hours prior to testing to minimize the increased urinary marking when mice are placed in a novel environment ${ }^{12}$. Animals were placed individually in standard cages for five hours $\left(332 \times 150 \times 130 \mathrm{~mm}\right.$ - floor area $\left.290 \mathrm{~cm}^{2}\right)$ with the floor lined with filter paper (Bench coat paper, VWR international, Amsterdam, The Netherlands). Animals were raised of the floor by $30 \mathrm{~mm}$ to avoid direct contact with the filter paper by placing a standardized metal walking grid in the cage (grid II44B-I50, Techniplast, Buguggiate, Italy) (Fig. I, panel A). Urinary markings on the paper were allowed to dry for 12 hours, after which they were illuminated with ultraviolet light, and photographed ${ }^{13}$. The experiment was recorded on video with a low level light from below illuminating the filter paper. For analysis of behaviour the cage was divided into three zones, the two corners were analysed together as corner zone and the third zone, the centre as the centre zone. The surface area of these zones was equal (Figure I, panel B).

The frequency and pattern of urinary markings were quantified within each zone, the volume for each marking calculated and the total distance moved and overall time spend in each zone recorded. In addition, the time point of each marking was recorded allowing easy identification and discrimination of different overlapping markings. In analogy with $\mathrm{Hu}$ et al. a linear relationship was found between spot area and the volume of the urinary marking, resulting in a ratio of $8.84 \mu \mathrm{l} / \mathrm{cm}^{2}$ (Figure 2) ${ }^{14}$. 
A

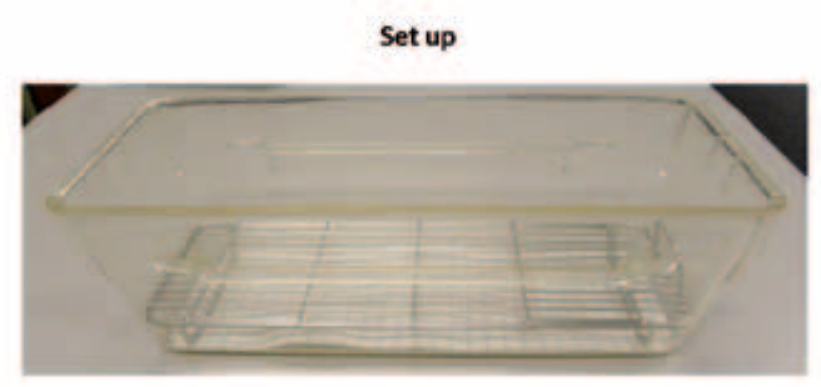

B

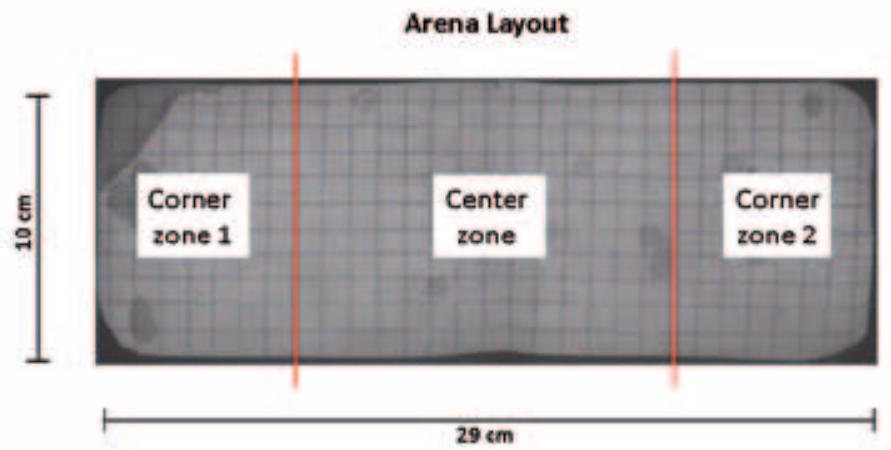

A

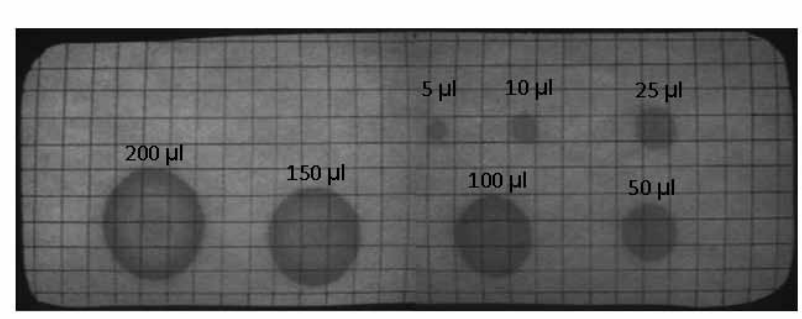

B

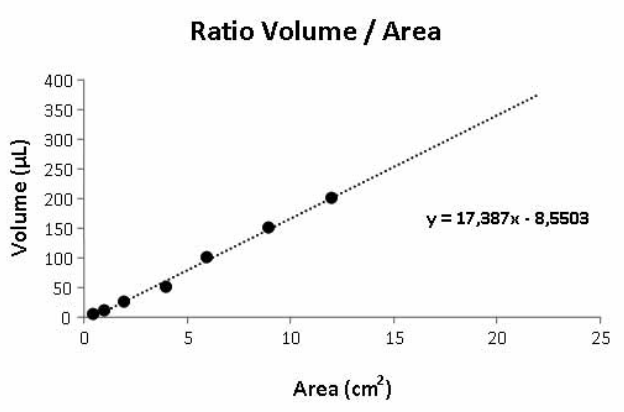

Figure I. Standard set up and arena layout for voiding behaviour task (VBT). (A) This photograph shows the cage, grid and filter paper as used in theVBT.The mouse is placed in the centre of the cage at the start of the experiment.A metal cage top closes the cage and provides for food and water. (B) Arena layout. UV photograph showing filter paper on the bottom of the conventional mouse cage.The floor of the cage measures $29 \mathrm{~cm}$ by $10 \mathrm{~cm}$; grid spacing is $1 \mathrm{~cm}^{2}$ in both directions. The total area of corner zones I and 2 combined equal the area of the centre zone.

\section{Figure 2. Volumetric} calibration. (A) UV photograph of the filter paper showing standardized volumes of urine with the corresponding area. (B) Scatter plot illustrating the relationship between volume and surface area of the urinary markings. The dotted line illustrated the linear trend (equation shown in the graph). 


\section{Elevated zero maze task}

The EZM was performed in order to study anxiety-related behaviour ${ }^{15}$. The test was carried out on a maze constructed of black plastic, transparent for infrared light. The circular runway was $50 \mathrm{~cm}$ in diameter, with a pathway width of $5 \mathrm{~cm}$ placed $20 \mathrm{~cm}$ above floor level. The maze was equally divided in two opposite open and two opposite closed parts enclosed by $50 \mathrm{~cm}$ high sidewalls. To prevent falls, a $5 \mathrm{~mm}$ high rim lined the open parts. A mouse was placed on one open arm, facing one of the closed arms of the maze and was allowed to explore the arena for five minutes. At the end of each experiment the surface was cleaned with $70 \%$ ethanol. Mice were tracked under low light conditions with an infrared video tracking system (Ethovision, Noldus, The Netherlands) to determine the total distance moved and time spent in zones (closed or open). The partitioning of time between open and closed regions was the principal index of anxiety/fearfulness.

\section{Open field task}

The OF was conducted to analyse spontaneous locomotor behaviour. The assessment was conducted in a square, Plexiglas box $(25 \mathrm{~cm} \times 25 \mathrm{~cm} \times 30 \mathrm{~cm})$, with an open top and grey floor. The floor of the arena was divided into centre, periphery, and corner zones, defined by lines spaced $5 \mathrm{~cm}$ from the sidewalls. A mouse was placed in the centre of the arena and allowed to move freely. The movements and position of the animals were recorded and registered automatically by a computerized system (EthoVision, Noldus, The Netherlands) in order to determine the time spent in the different zones and the total distance moved during the 10 minutes trial. After each experiment the surface was cleaned with $70 \%$ ethanol.

\section{Statistics}

All data are represented as mean and standard error of mean (SEM). All statistic analyses were done using the Statistical Package for the Social Sciences (SPSS I5.0 software, Chicago, IL, USA). Chi-square analysis was conducted for the VBT to compare the location of urinary markings within the groups. Between the groups, urinary markings, inter-marking interval were compared using a non-parametric Mann-Whitney $U$ test. The accepted level of statistical significance was determined on $\mathrm{p}<0.05$ for all analyses. The EZM and OF were analysed over time using the unpaired 2-sided Student $T$-test with location and distance moved as variables. One animal from the WT group was excluded from further analysis because of extreme anxiety-related behaviour in the EZM (continuous freezing). 


\section{Results}

\section{Voiding behaviour task}

There was no difference found in body weight between the groups (WT vs. APP/PSI; $25.8 \pm 1.3$ grams vs. $24.2 \pm$ I.I grams, $p=0.26 \mathrm{I}$ ).

Figure 3 shows photographs of filter paper of the voiding behaviour task (VBT) of APP/PSI mouse (A) or WT mouse (B). Under UV light the urinary markings were clearly visible as dark halos on a light background. In general, two types of urinary marking behaviour could be identified from the video tracking analysis. Common were non-circular markings seen in the corner zone, whereby the mouse (both WT and APP/PSI) pushed its backside against a wall of the cage and urinated (Figure 3 , * arrows). In contrast, circular markings occurred away from the walls and could be found anywhere in the cage. Video tracking analysis showed that they appeared when the mice made a short pause in their locomotion (Figure 3, \# arrows).

A

WT

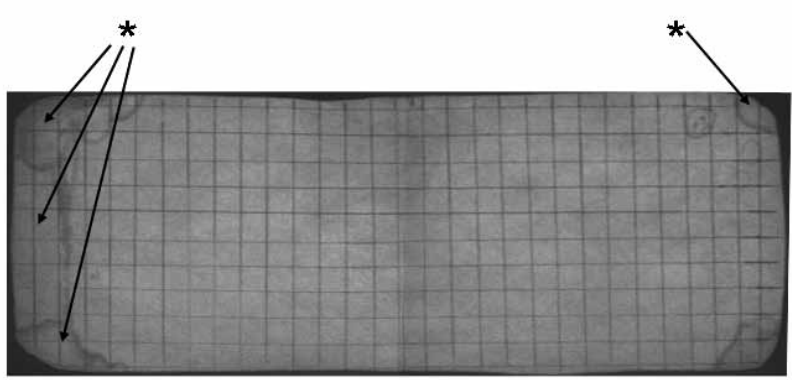

B

APP/PS1

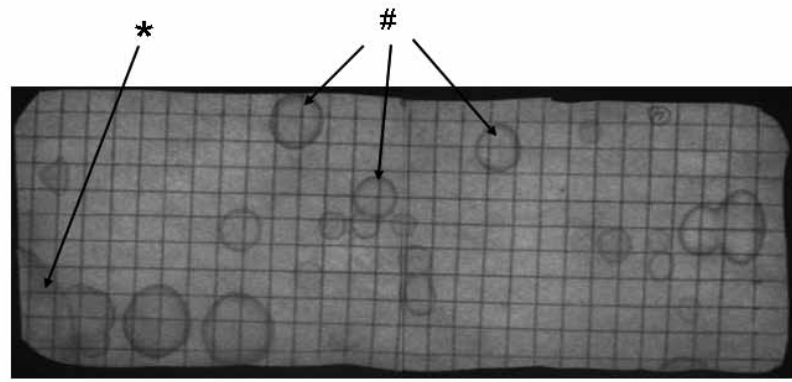

Figure 3. Representative photographs of a voiding behaviour task (VBT) filter paper of a WT mouse and an APP/PS I mouse. The VBT of the WT mouse has a distinct pattern with a high marking density in both corner zones (*arrows), whereas no urinary markings are visible in the centre zone. In the APP/PSI mouse urinary markings occur all across the floor area, in both corner zones but also in the centre zone (\# arrows). Similar to the WT mouse non-circular urinary markings were observed in the corner zones (*arrow). These results were representative for all other mice in both APP/PSI and WT groups. 
Upon analysis theVBT of the APP/PSI mice appeared significantly different fromWT mice. Firstly, the number of markings in the centre zone of the APP/PSI mice was significantly higher compared to theWT mice (Figure 4A,WT vs.APP/PSI; I.0 0.4 vs. $3.3 \pm 0.7, \mathrm{p}=0.009$ ). Conversely,APP/PSI mice urinated less frequently in the corner zone compared toWT mice (Figure 4A,WT vs.APP/PSI; 7.9 \pm 0.7 vs. $3.5 \pm 1.0, p=0.003)$. No difference was found between the individual mice of the APP/PSI group.

Secondly, the total volume of markings for the APP/PSI mice was significantly lower than for the WT mice (WT vs. APP/PSI; 548.9 $\pm 5.7 \mu \mathrm{L}$ vs. $348.7 \pm 9.4 \mu \mathrm{L}, \mathrm{P}=0.036$. In corner zone, the total volume of markings for WT mice was significantly larger than for APP/PSI mice whereas in centre zone, the opposite was observed (Fig. 4B, corner WT vs. APP/PSI; $508.7 \pm 6.2 \mu \mathrm{L}$ vs. $259.9 \pm 16.4 \mu \mathrm{L}, \mathrm{P}=0.014$ and centre; WT vs. APP/PSI; $40.2 \pm 10.4 \mu \mathrm{L}$ vs. $88.9 \pm 5.09 \mu \mathrm{L}, \mathrm{P}=0.28)$.

Concerning the average volume of a marking, in the WT group, no significant difference was observed between the corner zone and the centre zone (Figure 4D, corner vs. centre; $64.4 \pm$ $6.2 \mu \mathrm{L}$ vs. $40.2 \pm 10.4 \mu \mathrm{L}, \mathrm{P}=0.18)$. Interestingly, in the APP/PSI group, the average volume of a marking in the corner zone was also significantly greater than in the centre zone (Fig. 4D, corner vs. centre; $74.2 \pm 16.4 \mu \mathrm{L}$ vs. $26.7 \pm 5.1 \mu \mathrm{L}, \mathrm{P}=0.009$ ), showing that although these mice urinated evenly throughout the cage, the larger voids were done in the corner zone.

No significant difference in the inter-marking interval was observed between the groups, initially the frequency of marking was relatively high, after which it gradually decreased (Figure 4C). This could indicate some recurrent scent marking behaviour, e.g. re-familiarisation with the environment of the cage ${ }^{12}$. 
A

Number of spots in zone

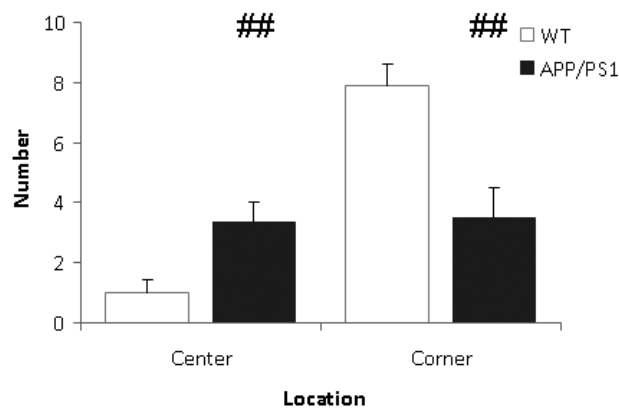

C

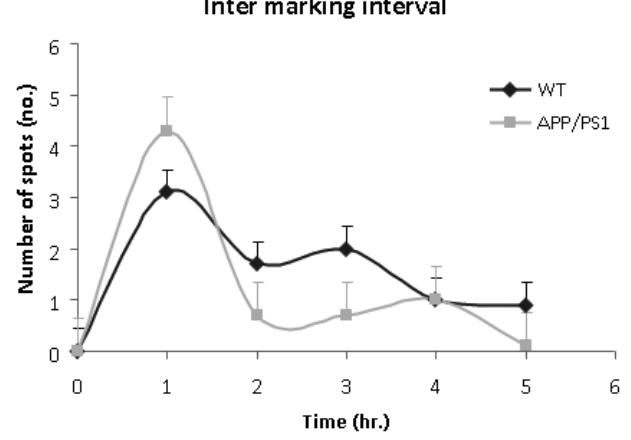

B

Total volume of spots in zone

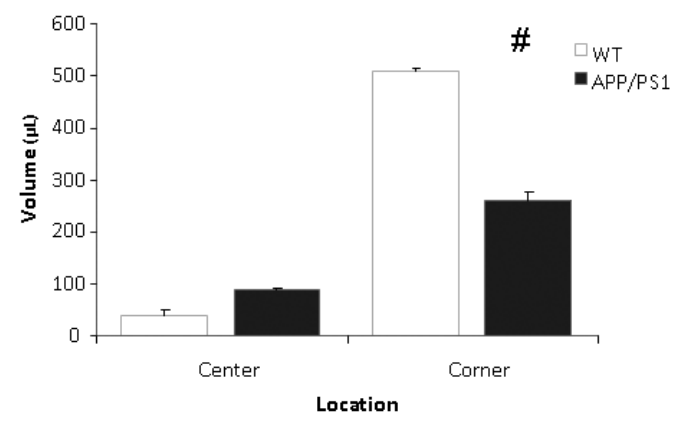

D

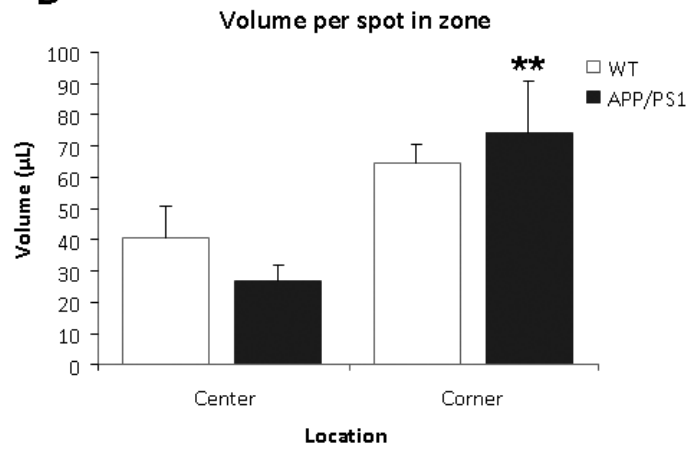

Figure 4. Voiding Behaviour Task (VBT); Voiding parameters. (A) The average number of markings in a zone. In corner zone, the number of urinary markings was significantly higher for the WT mice than the APP/PSI mice. The APP/ PSI mice urinated significantly more in the centre zone compared to the WT mice. Values are represented as means \pm SEM for the number of animals analysed (WT $n=9$ and APP/PSI $n=7$; different from WT \#\# $p<0.01$ ). (B) The total volume of urinary markings in a zone. Compared to APP/PSI mice, the volume of urinary markings for WT mice were larger in the corner zone but not in the centre zone. Values are represented as means \pm SEM for the number of animals analysed (WT $n=9$ and APP/PSI $n=7$; different from WT\# $p<0.05$ ). (C) The inter-marking interval shows the average number of markings per hour. No difference was observed between the groups, the frequency of micturition was relatively high initially and decreased gradually during the trial. Values are presented as means \pm SEM. (D) The average volume of a spot in a zone. In both centre and corner zones, there was no significant difference in average volume of urinary markings between the WT and APP/PSI mice. In APP/PSI group, the average volume of a marking in the corner zone was larger than in the centre zone. Values are represented as means \pm SEM (APP/PSI group, corner vs. centre, $\left.{ }^{* *} \mathrm{p}<0.0 \mathrm{I}\right)$. 
A

Time in zone

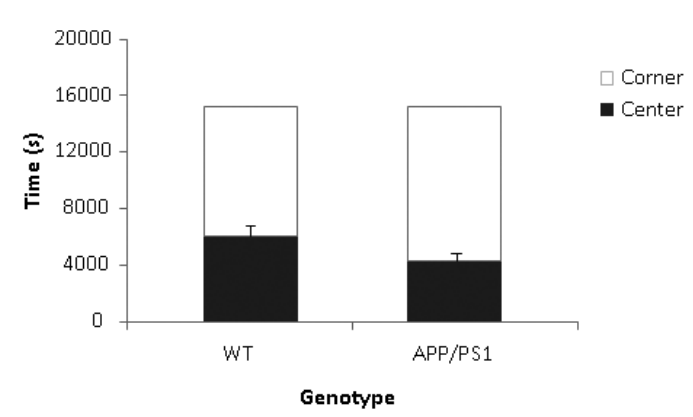

B

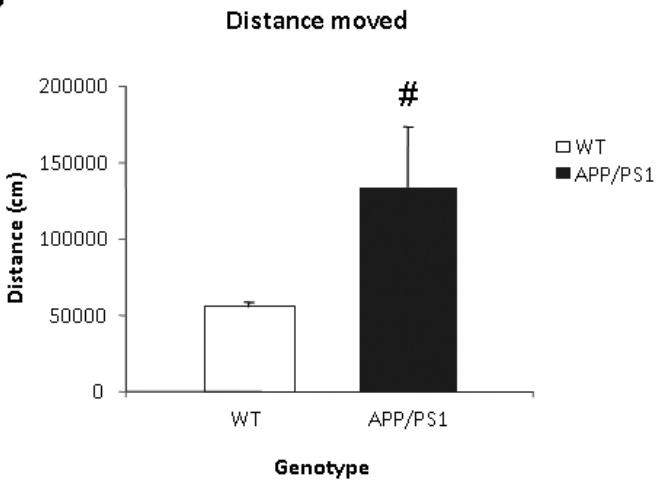

Figure 5. Voiding Behaviour Task (VBT); Behavioural parameters. (A) The time spent (in s) in the centre and corner zones of the VBT. There was no significant difference in time spent in the centre or corner zones between the WT or APP/PSI groups. (B) Mean total distance moved $(\mathrm{cm})$ in the VBT. The APP/PSI mice moved significantly more than the WT group. WT $n=9$ and APP/PSI $n=7$. Data represent means \pm SEM $(\# p<0.05)$.

Video tracking indicated that APP/PSI mice did not spend significantly more time in the centre than the WT group (Figure 5A). However, the APP/PSI mice moved significantly more distance than the WT group (Figure 5B).

\section{Elevated zero maze task}

Anxiety related behaviour was examined in the elevated zero maze task (EZM). This task was conducted one day after the VBT. Data were expressed as time spent in the open arms. Significant differences were found between the groups in the time spent in the open arms. The WT mice spent significantly more time in the open arms of the arena compared to the APP/PSI mice $(P=0.012)$ (Figure 6A).

\section{Open field task}

General locomotor behaviour was examined using the open field task (OF) one day after the EZM task. The data were expressed as total distance moved. The APP/PSI mice covered significantly more distance compared to the WT mice $(p=0.002)$ (Figure 6B). 
A

Elevated Zero Maze

Time in Open Arms

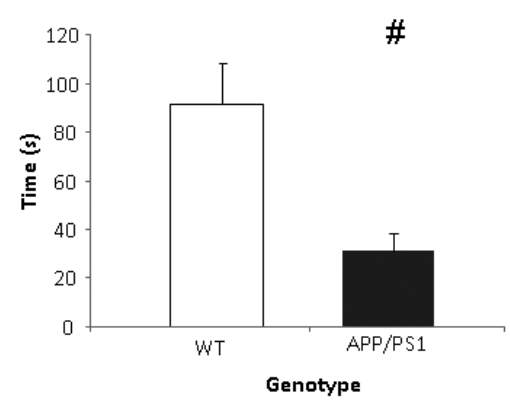

C

Time in zone

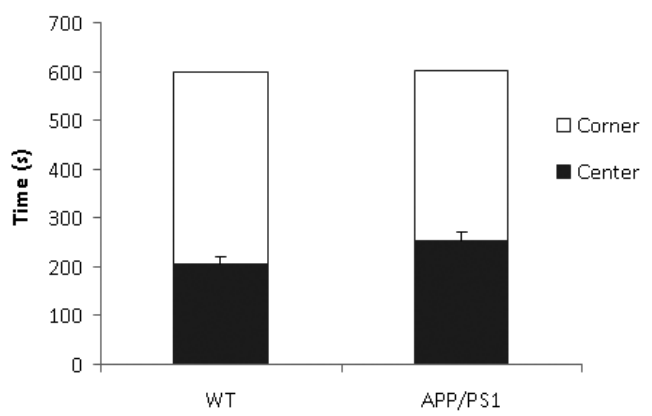

B

Open Field

Distance moved

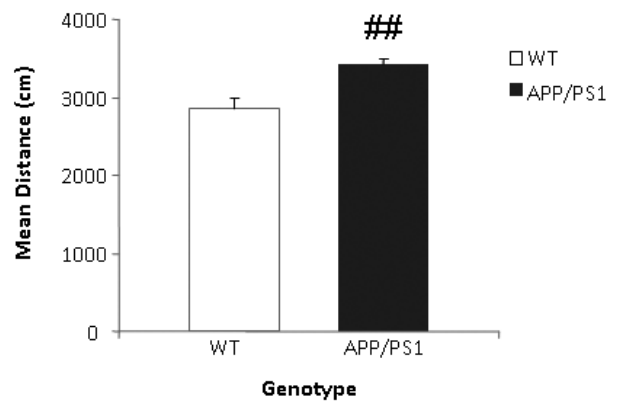

Figure 6. Elevated Zero Maze (EZM) and Open Field (OF) tasks. (A) The time spent (in s) in the open arms of the EZM. The WT group spends significantly more time in the open arms of the arena $(\# p<0.05)$. (B) The mean total distance moved (in $\mathrm{cm}$ ) in the OF. The APP/PSI mice moved more than the WT mice. WT $\mathrm{n}=9$ and APP/PSI $\mathrm{n}=7$. Data represent means \pm SEM (\#\# $\mathrm{p}<0.01)$. 
Structural and Functional Aspects of Sensory-motor Interaction in the Urinary Bladder

\section{Discussion}

The association of Alzheimer disease (AD) and voiding disorders has been known for a long time $^{16}$. Currently, no animal data are available linking Alzheimer's AD to voiding behaviour and/or bladder dysfunction. In this study we have focused on urinary marking, anxiety-related and general locomotor behaviour of a mouse model of AD. To our knowledge this is the first study to describe an altered voiding behaviour in a rodent model of $A D$.

Our results show that the WT mice demonstrated a well-controlled and organized marking pattern with markings occurring almost exclusively in the corner zones of the cage, creating the non-circular marking, a pattern considered to be normal ${ }^{17}$. In contrast, the APP/PSI mice showed circular markings in the centre of the cage. This evenly distributed marking pattern indicates the APP/PSI mice have no specific preference for location of urination (Figure 3). As urgency urinary incontinence is known to occur in $\mathrm{AD}^{4}$, we hypothesize that these markings could also be a sign of abnormal urination, possibly indicating some form of urinary incontinence.

The results of the EZM show that APP/PSI mice have elevated anxiety levels, since they spent less time in the open arms of the maze. These APP/PSI mice are known to have cognitive deficits as well ${ }^{10}$, which might contribute to the observed increased anxiety. Importantly, anxiety has been described as one of the most frequent and severe behavioural disturbances in AD and is associated with voiding dysfunction ${ }^{4,5}$. Consequently, increased anxiety levels might therefore explain the altered voiding behaviour of the APP/PSI mice.

In accordance with Vanmierlo et al., we also found that the APP/PSI mice moved a significantly larger distance in the arena during both the VBT and the OF tasks ${ }^{10}$. This locomotor behaviour in APP/PSI mice could be similar to the increased wandering behaviour as observed in AD patients ${ }^{18}$. Interestingly, the regions in the brain responsible for locomotor control, e.g. the basal ganglia and the medial frontal lobe, overlap with the frontal micturition centre. Lesions in these basal ganglia cause both motor and micturition disorders, a combination which is also seen in AD patients ${ }^{19}$.

Unexpectedly, in this study, the APP/PSI mice voided less than WT mice. This could be due to several factors; e.g. increased anxiety suppressing the APP/PSI mice to void, decreased fluid intake due to behavioural impairment resulted in reduce urine production.

Another possible explanation is that structural changes in the neural control mechanisms of the lower urinary tract could give rise to aberrant afferent activity leading to an altered voiding behaviour. Interestingly, our group has described histological changes in the urinary bladder of the APP/PSI mice indicating changes at the peripheral level ${ }^{7}$. In these mice, ganglia were found on an aberrant location, in the bladder wall itself, and the number of intramural afferent nerve fibers was increased ${ }^{7}$. How and when these changes occurred is still unclear and subjects to further research. In summary, the current study shows an altered voiding behaviour in a mouse model of $A D$. These alterations in the APP/PSI mice could be explained by changes in anxiety-related and general locomotor behaviour, specific of AD. Indeed previous studies suggest that behavioural changes are an important factor in incontinence in $\mathrm{AD}^{2}$. However, we hypothesize that the altered voiding 
behaviour is due to multi-factorial changes in behaviour as well as in the control of the urinary system leading to "functional incontinence". To some extend the altered voiding behaviour could be caused by an increased level of afferent activity of the lower urinary tract. The mechanisms remain largely unclear but are most likely caused by dysfunctions at both central and peripheral levels of control.

For future research, the non-invasive method of evaluating voiding behaviour used in this study should be combined with functional experiments, using cystometry. This can give more insight into the function and functional changes of the bladder in this animal model. Moreover, a newly developed rat model of AD could be an interesting model in terms of translational research to the human condition ${ }^{20}$. These models will be useful to elucidate whether functional changes develop first at the peripheral level or at the central level, i.e. due to changes in the bladder itself and/or in the brain in AD. The exact underlying mechanism between AD and altered voiding behaviour needs to be elucidated in future research. 
Structural and Functional Aspects of Sensory-motor Interaction in the Urinary Bladder

\section{References}

I. Ransmayr, G. N., Holliger, S., Schletterer, K. et al.: Lower urinary tract symptoms in dementia with Lewy bodies, Parkinson disease, and Alzheimer disease. Neurology, 70: 299, 2008

2. Han, D., Wang, Y.: Urinary Incontinence in Dementia. Incont Pelvic Floor Dysfunct, 2: 63, 2008

3. Jirovec, M. M., Wells, T.J.: Urinary incontinence in nursing home residents with dementia: the mobilitycognition paradigm. Appl Nurs Res, 3: I12, 1990

4. Serra, L., Perri, R., Fadda, L. et al.: Relationship between cognitive impairment and behavioural disturbances in Alzheimer's disease patients. Behav Neurol, 23: 123, 2010

5. Fan, Y. H., Lin, A. T., Wu, H. M. et al.: Psychological profile of female patients with dysfunctional voiding. Urology, 7I: 625, 2008

6. Sugiyama, T., Hashimoto, K., Kiwamoto, H. et al.: Urinary incontinence in senile dementia of the Alzheimer type (SDAT). Int J Urol, I: 337, 1994

7. Biallosterski, B. T., de Wachter, S. G., van Koeveringe, G. A. et al.: Changes in bladder innervation in a mouse model of Alzheimer's disease. J Chem Neuroanat, 39: 204, 2010

8. Stechman, M. J., Ahmad, B. N., Loh, N. Y. et al.: Establishing normal plasma and 24-hour urinary biochemistry ranges in $\mathrm{C} 3 \mathrm{H}, \mathrm{BALB} / \mathrm{c}$ and $\mathrm{C} 57 \mathrm{BL} / 6 \mathrm{~J}$ mice following acclimatization in metabolic cages. Lab Anim, 44: 218, 2010

9. Wood, R., Eichel, L., Messing, E. M. et al.: Automated noninvasive measurement of cyclophosphamideinduced changes in murine voiding frequency and volume. J Urol, 165: 653, 2001

10. Vanmierlo, T., Rutten, K., Dederen, J. et al.: Liver $X$ receptor activation restores memory in aged AD mice without reducing amyloid. Neurobiol Aging, 32: 1262, 2011

II. Kohler, C., Ebert, U., Baumann, K. et al.: Alzheimer's disease-like neuropathology of gene-targeted APP-SLxPSImut mice expressing the amyloid precursor protein at endogenous levels. Neurobiol Dis, 20: 528, 2005

12. Maruniak, J. A., Owen, K., Bronson, F. H. et al.: Urinary marking in male house mice: responses to novel environmental and social stimuli. Physiol Behav, 12: 1035, 1974

13. Desjardins, C., Maruniak, J. A., Bronson, F. H.: Social rank in house mice: differentiation revealed by ultraviolet visualization of urinary marking patterns. Science, 182: 939, 1973

14. Hu, P., Deng, F. M., Liang, F. X. et al.: Ablation of uroplakin III gene results in small urothelial plaques, urothelial leakage, and vesicoureteral reflux. J Cell Biol, I5I: 961, 2000

15. Shepherd, J. K., Grewal, S. S., Fletcher, A. et al.: Behavioural and pharmacological characterisation of the elevated "zero-maze" as an animal model of anxiety. Psychopharmacology (Berl), I16: 56, 1994

16. Williams, M. E., Pannill, F. C., 3rd: Urinary incontinence in the elderly: physiology, pathophysiology, diagnosis, and treatment. Ann Intern Med, 97: 895, 1982

17. Gevaert, T., Vriens, J., Segal, A. et al.: Deletion of the transient receptor potential cation channel TRPV4 impairs murine bladder voiding. J Clin Invest, II7: 3453, 2007

18. Burns, A., Jacoby, R., Levy, R.: Psychiatric phenomena in Alzheimer's disease. IV: Disorders of behaviour. Br J Psychiatry, 157: 86, 1990 


\section{Changes in Voiding Behaviour in a Mouse Model of Alzheimer's Disease}

19. Garcia-Rill, E.: The basal ganglia and the locomotor regions. Brain Res, 396: 47, 1986

20. Cohen, R. M., Rezai-Zadeh, K., Weitz, T. M. et al.: A transgenic Alzheimer rat with plaques, tau pathology, behavioral impairment, oligomeric abeta, and frank neuronal loss. J Neurosci, 33: 6245, 2013 



\section{Chapter 7 \\ General Discussion}

\section{Study Purpose}

The aims of the studies presented in this thesis are to gain a better understanding of structural and functional aspects of the sensory information and the motor output of the urinary bladder. Several experiments have been carried out for this purpose and can be divided in two main sections of this thesis. The first part focuses on the morphological and structural changes in the urinary bladder wall of the guinea pig, addressing the spatial relationship and distribution of the prostaglandin E receptor type 2 (EP2) in the guinea pig urinary bladder and the different subtypes of afferent nerve fibres in the mouse bladder wall.

In the second part, the studies concentrate on functional bladder studies in mice. The effect of filling rate and intravesical filling on non-voiding contractions, together with the differences in the structure of bladders from transgenic mouse models of Alzheimer's disease with age matched control animals were studied. In addition, the urinary marking behaviour in relation to affective behaviour in an Alzheimer model mouse has been evaluated.

The sensation of urge in most people is a signal to which pay attention can be paid to or that can be ignored for a while. Sadly however, this is not so for a significant number of people. They have sensations that can completely dominate their life, often driving them to seek help. These patients present with, urgency, the sensation of a sudden compelling desire to void that is difficult to defer, often accompanied by urinary frequency and urinary incontinence eventually nocturia' (more than 8 micturitions in 24 hours). This symptom complex is called the overactive bladder syndrome (OAB) ${ }^{1-3}$. The underlying causes of $O A B$ remain unclear. In about $50 \%$ of patients $O A B$ is accompanied by detrusor overactivity (DO) which is defined as uncontrolled contractions of the bladder muscle during bladder filling (motor $O A B)^{4,5}$. The sensory OAB has the same symptoms but without DO shown on conventional urodynamic studies ${ }^{54,55}$.

The incidence of $O A B$ increases with age ${ }^{6}$ and because of an aging society in the Western world, $O A B$ is becoming a significant and prevalent problem. Moreover, elderly individuals especially with neurological diseases such as dementia and Alzheimer's disease become more prevalent and often have $O A B$ symptoms which reduce their quality of life and increase the complexity of their care.

Since $O A B$ symptoms have been shown to be associated with detrusor overactivity, it was concluded that drugs affecting contractility would alleviate symptoms ${ }^{16}$. Activity in the bladder smooth muscle is initiated by muscarinic receptor stimulation. The mainstay of current treatments is antimuscarinics, which have a better effect than placebo, but poor patient compliance, due to their side effects and the lack of sufficient clinical efficacy ${ }^{7,8}$. Therefore, it is desirable that alternative treatment methods are developed and made available to patients. Both the prevalence of OAB and 
Alzheimer's disease increase with age, and also the number of elderly people increases. Moreover, many patients with Alzheimer's disease have urinary incontinence and OAB symptoms. A better understanding of the structural and functional aspects of the sensory-motor interaction in the urinary bladder and the changes in bladder morphology and behaviour in Alzheimer's disease, could bring about a better understanding of the underlying pathological mechanisms and hopefully will be a focus for better future treatment modalities.

\section{Animal Models}

Legal, ethical and moral issues prevent that experiments, crucial for the understanding of the mechanisms involved in the bladder can be carried out in human subjects. Moreover, human bladder tissue is difficult to gain, especially from patients affected with Alzheimer's disease. Therefore, we have used the guinea pig bladder as our study model. The guinea pig bladder has similarities in structure as well as comparable bladder cystometric parameters to the human bladder ${ }^{9-12}$ and has been used extensively in our and other laboratories ${ }^{13-16}$. The use of the same animal model for experiments makes comparison of data easier. Therefore, the guinea pig bladder was the first choice for the experiments as presented in this thesis.

In addition, in order to study specific changes in Alzheimer's disease, we have used a transgenic mouse model. In this mouse model for Alzheimer's disease, related pathology has been mimicked without over-expression of the amyloid precursor protein (APP ${ }^{S L}$ ) but dependent on the co-expression of mutant human presenilinI protein (PSI $\left.{ }^{\mathrm{I} I 46}\right)^{17}$. Gene-targeted mice display a similar pattern of plaque distribution and show similar features of Alzheimer disease-like pathology, like glial reaction around compact plaques, dilated cholinergic terminals in the cortex, and the absence of overt neuro-degeneration ${ }^{17}$.

\section{The role of Prostaglandin in the bladder and the choice of studying EP2}

From all prostanoids, $\mathrm{PGE}_{2}$ has been put forward as the most likely candidate to contribute to bladder overactivity. ${ }^{18}$ This is due to the previous finding that $\mathrm{PGE}_{2}$ infused into the bladder reduces bladder capacity. ${ }^{19-22}$. We chose to study EP2 receptor, as EPI, EP3 and EP4 immunohistochemical characterization in the bladder had been addressed before ${ }^{13,23,24}$. The EP2 receptor distribution in the guinea pig urothelium was already explored ${ }^{14}$, but EP2 receptor distribution in the muscle layer was not studied yet using immunohistochemical techniques. Therefore, we designed experiments, using visualisation techniques to pinpoint the target and production site of PGs in order to answer our first research question on the localization and the role of EP2 receptors in the bladder and on the possible use of these receptors as a target in the treatment of lower urinary tract dysfunctions. 


\section{EP2 receptor expression in the detrusor}

The EP2 receptor is expressed in the urothelium and in the sub-urothelial interstitial cells (SU-ICs) ${ }^{14}$. In our study, we have shown that EP2 receptors are also expressed on intramuscular interstitial cells (IM-ICs) and surface muscle interstitial cells (SM-IC's) of the inner- and the outer-muscle layer. In addition, our results show a punctuate low intensity expression of EP2 in guinea pig detrusor muscle bundles. This punctuate appearance deserves more future attention as to whether this might be due to certain areas serving a specific function, for example in cell to cell contacts.

In the muscle, each of the EP receptors is known to act through different intracellular pathways that involve calcium transport and the stimulation or inhibition of adenylate cyclase to elicit muscle contraction or relaxation depending on the EP subtypes ${ }^{25}$. In general, EPI and EP3 are thought to cause contraction of the smooth muscle, whereas EP2 and EP4 are thought to cause relaxation ${ }^{26,27}$. Networks of IC's with short and long processes, are visible from the urothelium and sub-urothelium through the muscle layers and form connections with other IC's nodes. There is a clear COX I expression in the basal urothelial layer as also reported in earlier papers ${ }^{13,14}$, suggesting the possibility that a PG signal, presumably mediated and enhanced by ATP release from the urothelium can be picked up by EPI receptors and be spread further via the IC network ${ }^{13,14}$. Our data demonstrate COX I and EP2 immuno-reactivity, in close proximity to each other or even co-expressing within the same cell. Further research is necessary in order to determine the physiological role of this EP2 receptor configuration. However, a spread and augmentation of a signal mediated by PG via EP2 is unlikely as in most smooth muscle preparations, other than the urinary bladder, EP2 has been shown to cause muscle relaxation ${ }^{26}$.

\section{Sub-populations of afferent nerve fibres}

Through experiments carried out in mouse bladders, we explored the immunohistochemical staining pattern for the most prevalent afferent nerve fibres which might reflect a functional differentiation. Furthermore, regional differences between the lateral wall and bladder base were studied.

\section{Calcitonin gene related peptide (CGRP)}

CGRP immune reactive axonal nerve fibre endings are located at several positions in the urothelium including the basal layer, the urothelial epithelial cells and even right underneath the umbrella cells, characterizing them as primary afferent neurons. These nerve fibres are likely to be afferent C-fibres. These fibres have been shown to be responsive to capsaicin, releasing CGRP into the bladder wall, after activation of the transient receptor potential cation channel subfamily $\mathrm{V}$ member I receptor (TRPVI) ${ }^{28}$. Although the cellular location of the TRPVI in the bladder is still under debate, but recent research has shown co-localization with CGRP indicating it's presence on afferent nerve fibres extending into the urothelium ${ }^{29}$. The most likely location is in the urothelial cells ${ }^{30}$. Because CGRP has been found to inhibit the phasic activity in the isolated bladder preparation these fibres 
Structural and Functional Aspects of Sensory-motor Interaction in the Urinary Bladder

could be part of a motor/sensory system ${ }^{31}$. This is emphasized by the fact that deletion of the TRPVI receptor in knock out mice resulted in diminished afferent responses to mechanical stimuli ${ }^{32}$.

Protein gene product 9.5 (PGP 9.5)

PGP immuno-reactive nerves were uniformly present in the muscle layers of both bladder base and lateral wall. At the lateral wall the lamina propria was sparsely innervated with PGP-IR nerve fibres compared to the bladder base. Most of the PGP 9.5 and CGRP immuno reactive nerve fibres run close to each other in the sub-urothelial space, which raises the possibility of direct exchange of impulses between the nerve fibres.

\section{Neuro filament (NF)}

NF immune-reactive nerve fibres were not distributed throughout all layers of the bladder wall. In this study NF is used as a marker for $A \delta$ nerve fibres since in the dorsal root ganglia (DRG) NF is exclusively expressed in myelinated $\mathrm{A} \delta$-fibres, and not in unmyelinated $\mathrm{C}$-fibres ${ }^{33}$. However, the majority of bladder afferent nerve fibres is NF-negative ${ }^{34}$. In the muscle layers, these fibres are likely to be mechanosenstive responding to the slow filling of the bladder ${ }^{35}$. On the serosal side of the bladder base, both CGRP-immuno reactive afferent and NF-immuno reactive afferent axons probably project to ganglia were there signals are modulated and re-directed ${ }^{36}$.

Vesicular acetylcholine transporter (VAChT)

Another class of afferent fibre running near the urothelium were the VAChT-immunoreactive, cholinergic fibres, running close to the basal cell and the urothelial epithelial cell layers. As with all nerve fibres running near the urothelium, VAChT positive nerve fibres were considered to be afferent. As hypothesis proposed by Gosling and Dixon states that, "a sensory role can reasonably be proposed for those peripheral nerves whose terminals are regularly observed to be unrelated to recognized neuro-effector target sites" ${ }^{37}$. Moreover, in the rat, cholinergic nerve fibres in the lamina propria, expressing acetylcholine-esterase, have been found to co-localize with CGRP within the urothelium of ureters ${ }^{38}$. The lamina propria at the bladder base was more densely innervated with VAChT-IR nerve fibres, in parallel with the distribution of CGRP-IR nerve fibres. These regional variations in innervation of the bladder base and the lateral wall have been described in the rats and in humans as well ${ }^{39,40}$. The muscle layers were uniformly innervated by VAChT positive nerve fibres, where this marker could identify both afferent and efferent fibres ${ }^{41,42}$.

Neuronal Nitric oxide synthases (nNOS)

Nerve fibres containing nNOS were distributed throughout the bladder but predominated in the bladder base. Most NOS-containing (nitrergic) nerve fibres also contain VAChT and can therefore be considered cholinergic ${ }^{43}$. These nerve fibres were primarily found in the muscle layers with few nerve fibres running in the suburothelial layer. Dixon et al. have shown that nNOS is sparse in cholin- 
ergic nerves of the lateral wall, but present in the majority of these nerves at the bladder base ${ }^{40}$. In the muscle the bulk of cholinergic fibres are presumed to be efferent motor neurons responsible for initiating and sustaining a detrusor contraction ${ }^{42}$. At the bladder base cholinergic fibres may release $\mathrm{NO}$ known to induce smooth muscle relaxation in the urethra by activating guanylate cyclase and increasing cyclic guanosine monophosphate (cGMP) levels in the smooth muscle cell ${ }^{44}$. The latter observation may reflect a neurotransmitter mediated mechanism preceding micturition. Cholinergic stimulation gives rise to relaxation of smooth muscle at the bladder neck as well as a contraction of the detrusor body ${ }^{45}$. The function of the $\mathrm{nNOS}$ nerve fibres found in the lateral wall is less clear since not the detrusor smooth muscle cells but interstitial cells have been seen to produce cGMP in response to $\mathrm{NO}{ }^{46,47}$. The role of these cells is still uncertain possibly playing a role in the generation of non-voiding activity seen during bladder filling ${ }^{48}$.

\section{Intramural Ganglia}

Ganglia were seen at the serosal side of the bladder; all were PGP-IR and most were also nNOS positive. Varicosities of CGRP and VAChT positive nerve fibres, possibly indicating nerve terminals, and NF-IR nerve fibres were running in close proximity to the ganglia. These ganglia could be relay stations transferring input received from afferent nerve fibres in the bladder to the CNS as suggested by similar observations in the guinea pig bladder and also seen in other organ systems ${ }^{36,49}$. In addition, ganglia could also function in local motor-sensory networks of the bladder. Efferent output of these ganglia could be directed towards the interstitial cells or the smooth muscle cells.

Together with the urothelium, these structures might play a role in the generation and modulation of non-voiding activity seen during bladder filling ${ }^{50,51}$.

Thus, in our studies, using immunohistochemistry several sub populations of afferent nerve fibres were recognized in the mouse bladder and regional differences were identified. Based on our working hypothesis, that all nerve fibres in the lamina propria are considered to be afferent, we found at least four different types of afferent nerve fibre; CGRP, NF, VAChT and nNOS positive fibres. At the bladder base the suburothelium was densely innervated by all nerve fibres types, indicating that bladder sensation is predominant at this location. The dense innervation of CGRP positive nerve fibres in the inner muscle at the lateral wall implicates a possible mechanosensitive role for these nerve fibres at this location. The NF immune-reactive nerve fibres are probably also mechanosensitive, as they were primarily found in the muscle layers. The majority of VAChT positive nerve fibres in the muscle will most likely be efferent nerve fibres responsible for the detrusor contraction. At the bladder base $\mathrm{nNOS}$ positive nerve fibres will most likely induce smooth muscle relaxation whereas nNOS immune-reactive nerve fibres found at the lateral wall probably modulate the activity of interstitial cells. The ganglia located at the serosal side of the bladder were all PGP positive and mostly $\mathrm{nNOS}$ positive. All nerve fibres types ran in close proximity suggesting that these ganglia function as local motor-sensory networks receiving input from multiple different afferent nerve 
Structural and Functional Aspects of Sensory-motor Interaction in the Urinary Bladder

fibres and directing efferent output to the CNS and the bladder wall. The sensory information that is relayed by nerve fibres to the CNS is suggested to be modulated by the local bladder control mechanisms such as non-voiding contraction activated sensation.

\section{Non-voiding activity}

For many years, the bladder was considered to be inactive and silent (e.g. without any transient pressure increases) during the filling phase. The presence of any detrusor activity during filling phase was considered to be pathological. Furthermore, it is known that involuntary detrusor contractions may lead to symptoms such as urgency, increased frequency of voiding and incontinence.

However, ambulatory urodynamics has shown non-voiding detrusor contractions during the filling phase in up to $69 \%$ of healthy volunteers ${ }^{52,53}$, suggesting that these contractions may be a normal physiological mechanism. Currently the differences in non-voiding contractions (non-voiding contractions) between normal and pathological states are unknown. In animal model experiments, nonvoiding contractions have been shown to occur in vitro in the isolated whole bladder preparation ${ }^{54}$. In our experiments in anaesthetized guinea pigs, we demonstrated non-voiding contractions during the filling phase in the bladder. In general, the non-voiding contractions progressively increase in amplitude and frequency as the bladder fills. In the awake rat different phases in non-voiding activity have been described ${ }^{55}$. In the guinea pig no phases could be identified or separated, except a phase without non-voiding contractions and a phase with non-voiding contractions. Furthermore, in the rat the frequency of non-voiding contractions decreases just before micturition, which is not observed in the guinea pig. It is not clear whether these different observations are due to differences in species or the use of anaesthesia. Ex vivo, the isolated whole guinea pig bladder also shows non-voiding contractions, which are small $(0,2-3 \mathrm{~cm} \mathrm{H} 20)$ and regular, and increase in frequency and amplitude as bladder volume increases ${ }^{54}$. Since the bladder is disconnected from the central nervous system, the generation and modulation of the non-voiding contractions must be an intrinsic property of the bladder wall. The current in vivo isovolumetric data show a phasic pattern in the frequency of non-voiding contractions. Small amplitude non-voiding contractions seem to be the source of these bursts of increased frequency. This phasic pattern in frequency is also seen with gradual filling of the bladder. Again, small amplitude non-voiding contractions are the source of this phasic pattern. Such a pattern is absent in the isolated whole bladder ${ }^{54}$. This suggests that it is evoked by spinal reflexes and might play a role in "sensing" bladder volume. In the isovolumetric data the frequency of large amplitude non-voiding contractions is stable and does not increase, whereas in the filling experiments the frequency of large non-voiding contractions gradually increases with increasing volume. Previous afferent recording studies have shown that non-voiding contractions may elicit phasic firing of bladder afferents, suggesting that this form of bladder activity might underlie some form of motor-generated sensory function ${ }^{56-60}$. Both ex vivo and in vivo analysis of bladder filling at physiological rates revealed at least two different types of afferent activity ${ }^{56,60,61}$. One group 
showed phasic firing, which mirrored spontaneous contractions, while the other showed a continuous increasing firing rate during filling as a function of stretch ${ }^{62,65,66}$. Filling at a higher physiological rate shows similar afferent firing patterns ${ }^{60}$. However, at supra-physiological filling rate all afferents displayed the same pattern of continuous increased firing as the bladder fills. As filling rate increased the sensitivity of afferents towards bladder filling decreased ${ }^{61,62}$.

In the guinea pig, using various stimuli several functionally distinct populations of afferents have been identified, activated by different mechanisms. These can be stretch-sensitive (muscle mechanoreceptors and tension-mucosal mechanoreceptor) or stretch-insensitive (mucosal mechanoreceptors and chemoreceptors) ${ }^{62,63}$. In vivo, two types of stretch-sensitive afferents were reported, one with firing linearly related to intravesical pressure, while the second type, firing in a phasic pattern, reached a plateau or even decreased at higher pressure ${ }^{60}$. This last type of afferent has been suggested to act like "volume receptors", sensing bladder distension ${ }^{64}$. This type of afferent fiber could be involved in the generation of small non-voiding contractions, underlying the phasic pattern as described in this study. In conclusion, this study shows that non-voiding contractions are present in the guinea pig bladder in vivo, and that small amplitude non-voiding contractions exhibit a specific frequency pattern.

\section{Major differences in bladders of transgenic model mouse for Alzheimer's disease} In a mouse model of Alzheimer's disease we studied the morphological changes of the bladder wall. Our study demonstrated structural differences in the bladder wall of the APP ${ }^{\text {SL/PSI }}{ }^{\text {MI }}{ }^{46 L}$ Alzheimer's mice compared to age matched controls.

The first observation is that the cholinergic innervation of the smooth muscle layers in the bladder wall, as shown by the VACHT immuno-reactivity, is not distinctly different between the App ${ }^{\mathrm{SL} /}$ PSIMI46L mice and age matched controls. This suggests that the ability of the bladder to contract will not be impaired in the transgenic mice allowing them to maintain normal voiding contractions. Therefore, the primary changes may not be directly associated with the efferent control of the bladder.

The second major observation is a clear increase in the number of $\mathrm{VAChT}^{+}$and $\mathrm{nNOS}^{+}$nerve fibres within the lamina propria. It is generally assumed that nerves in this region of the bladder wall are sensory fibres contributing to the afferent output of the bladder. If this is so, the presence of $\mathrm{VAChT}^{+}$ and $\mathrm{NOS}^{+}$fibres in the ApP ${ }^{\mathrm{SL}} / \mathrm{PSI} \mathrm{II}^{\mathrm{MI} 6 \mathrm{~L}}$ mice suggest alterations in the afferent system. The physiological consequences of such changes are not known but a higher density of afferent nerves might result in a different processing in or different sensory outflow from the bladder. There are several different types of afferent fibres emanating from the bladder ${ }^{60,64-66}$. Some of these fibres are activated during normal bladder filling, whereas others may be activated by 'noxious' stimuli and may contribute to detrusor overactivity and overactive bladder symptoms ${ }^{67}$. It is still unclear which fibres only contribute to spinal reflexes and which give rise to conscious perception ${ }^{68}$. The altered expression of the VAChT and nNOS fibres in the lamina propria raises the interest to study the distribution 
Structural and Functional Aspects of Sensory-motor Interaction in the Urinary Bladder

of other nerve fibre types. For example Calcitonin Gene Related Peptide (CGRP) and transient potential receptor vanilloid I (TRPVI) fibres would be good candidates to study because they have been implicated in the occurrence of overactive bladder symptoms and Alzheimer patients have an increased prevalence of overactive bladder symptoms ${ }^{69-72}$. However, from immunohistochemistry studies we cannot say anything about the function of the putative afferent fibres in the APP ${ }^{\text {SL/PSIMI }}$ (46L $^{-}$ mice. The afferent fibres, contributing to the perception of bladder volume, are a sub-set of sensory fibres ${ }^{68}$. A similar heterogeneity in the nerves within the lamina propria and specifically associated with the urothelium has been reported in the normal guinea pig bladder ${ }^{36}$.

As a third observation, it can be noted that VAChT-positive fibres seen in the ApP ${ }^{\text {SL/PSIMI46L mice }}$ may be making contact with intramural ganglia. This anatomical arrangement has led to the suggestion that there may be local reflexes operating in the bladder wall ${ }^{36,73}$. Since there are no intra-mural ganglia in the normal mouse or rat bladder the concept of such local intra-mural reflexes in these species has not been considered ${ }^{74}$. There is, therefore, an important question regarding when and how the nerve cell bodies and ganglia appear in the APP ${ }^{S L} / \mathrm{PSI}^{\mathrm{MI} / 46 \mathrm{~L}}$ mice.

We can conceive at least two possibilities. It may be that the genetic changes leading to this aberrant generation of the neurones occur at an embryonic stage and persist into adult life. This possibility should be explored in the near future. Secondly the nerves and ganglia may generate later in life along with the other changes seen in these animals associated with Alzheimer's disease. De novo generation of neurones is not a common phenomenon and therefore this explanation seems less likely and at this stage it is not plausible. Contrary to this already present sleeping nerves and connections can be switched on in life due to a different transmitter balance or an adaptation to a new longer lasting bladder behaviour due to a different central nervous system control induced by Alzheimer's disease. Nerve cell bodies and small ganglia are found in the bladder in humans, pigs and guinea pigs ${ }^{36,47,75,76}$. Single nerve cell bodies are found but more often they are observed in small groups. The observation that they are associated with nerve fibres with terminal like structures has led to the suggestion that they receive synaptic inputs ${ }^{36,45,47}$. The presence of cholinergic terminals might imply that the intra-mural ganglia may receive both excitatory and inhibitory input where they may be a part of a motor/sensory system ${ }^{54,77}$. The properties and physiological role of these cells seen in the bladders of the APP ${ }^{S L} / P S I^{M 146 L}$ mice are not yet clear. They may represent small intra-mural ganglia and perform a motor role similar to that proposed in the guinea pig ${ }^{36}$.

From this data we cannot infer anything about the origin or development of these differences nor can we deduct anything about their physiological consequences or extrapolate these findings to the human conditions of dementia or Alzheimer's. However, there are differences in the afferent and possibly the motor-sensory system in the mouse model, which may have some importance. These findings may aid in elucidating some of the underlying pathology for the lower urinary tract dysfunction in humans with dementia and Alzheimer's disease and illustrate the importance of the peripheral nervous. They may also help to stimulate research on changes in the peripheral nervous 
system in animals models initially designed to study disease of the central nervous system. Structural immunohistochemical studies of bladder tissue of patients with Alzheimer's disease and physiological studies on the present mouse model is a next step to take.

\section{Changes in voiding behaviour in Alzheimer model mice}

The association of Alzheimer's disease and voiding disorders has been known for a long time ${ }^{78}$. This may partly be due detrusor overactivity as seen in patients with Alzheimer's disease, which may reflect increased sensation, e.g. urgency ${ }^{79}$. This however, is difficult to study due to cognitive impairment, which may also be a factor of incontinence, and ethical constraints. On the other hand, here is still no animal data available linking Alzheimer's disease to voiding behaviour and/or bladder dysfunction that can be seen in humans with Alzheimer's disease. This study on voiding behaviour in a mouse model of Alzheimer's disease, focused specifically on urinary marking and anxiety-related and general locomotor behaviour. We are the first to describe an altered voiding behaviour in a rodent model of Alzheimer's disease.

Our results show that wild type mice demonstrated a well-controlled and organized marking pattern. These animals showed marking patterns almost exclusively in the corner zones of the cage, creating the non-circular marking, a pattern considered to be normal ${ }^{80}$. In contrast, the

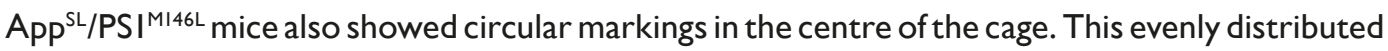
marking pattern indicated they had no specific preference for their location of urination. Since no descriptive data are available on this and urge incontinence is known to occur in Alzheimer's disease $^{81}$ we hypothesize that these markings could also be a sign of abnormal urination, possibly indicating some form of urinary incontinence or at least an aberrant voiding pattern.

The results of the elevated zero maze task show that APP ${ }^{S L} / P S I^{M I 46 L}$ mice have elevated anxiety levels, since they spent less time in the open zone of the maze. Our APP ${ }^{\text {SL/PSIMI46L }}$ mice are known to have cognitive deficits as well ${ }^{82}$, which might contribute to the observed increased anxiety. Importantly, anxiety has been described as one of the most frequent and severe behavioural disturbances in Alzheimer's disease and is associated with voiding dysfunction ${ }^{81,83}$. Consequently, the altered voiding behaviour of the APP ${ }^{S L} / P S I^{M / 46 L}$ might therefore be explained by increased anxiety levels.

In accordance with Vanmierlo et al., we also found that the ApP ${ }^{\text {SL/PSI }}{ }^{\mathrm{MI}}{ }^{46 \mathrm{~L}}$ genotype moved a significantly larger distance in the arena of both the voiding behaviour task and the open field locomotor behavioural task ${ }^{82}$. This locomotor behaviour in APP SPSI $^{\text {MI }}{ }^{\text {SLL }}$ mice could be similar to the increased wandering behaviour as observed in patients with Alzheimer's disease. Interestingly, the regions in the brain responsible for locomotor control, e.g. the basal ganglia and the medial frontal lobe, overlap with the frontal cortical micturition centre. Lesions in the basal ganglia cause both motor and micturition disorders, a combination which is also seen in Alzheimer's disease. In contrast to what we expected the APP ${ }^{S L} / P S I^{M / 46 L}$ mice in our study voided 
less frequently than wild type mice, this could be due to several factors; e.g. increased anxiety suppressing the APP ${ }^{\text {SL/PSIMI46L }}$ mice to void, decreased fluid intake due to behavioural impairment resulted in reduce urine production.

Another possible explanation is that structural changes in the neural control mechanisms of the lower urinary tract could give rise to aberrant afferent activity leading to altered voiding behaviour. This could be due to the loss of $\mathrm{MI}$ and $\mathrm{M} 2$ cholinergic processing in Alzheimer's disease both at the central and the peripheral level. Centrally, $\mathrm{MI}$ and $\mathrm{M} 2$ receptors are necessary for cognitive processing in the hippocampus and the cortex ${ }^{84}$. In the lower urinary tract $M 2$ receptors are present on smooth muscle in the bladder and $M I$ receptors are present on the parasympathetic nerve terminals at the neuromuscular junction and the parasympathetic ganglia responsible for afferent processing ${ }^{85}$. Interestingly, our group has described histological changes in the urinary bladder of the APP ${ }^{S L} / P S I^{M I 46 L}$ genotype indicating changes at the peripheral level ${ }^{86}$. In these mice, ganglia were found on an aberrant location, in the bladder wall itself, and the number of intramural afferent nerve fibres was increased ${ }^{86}$. How and when these changes occurred is still unclear and subject to further research.

The current study shows an altered voiding behaviour in a mouse model of Alzheimer's disease. These alterations could be explained by changes in anxiety-related and general locomotor behaviour in the APP/PSIMUT mice specific of Alzheimer's disease. Indeed previous studies suggest that behavioural changes are an important factor in incontinence in $A D^{87}$. However, we hypothesize that the altered voiding behaviour is due to multi-factorial changes in behaviour as well as the control and even the structure of the urinary system leading to "functional incontinence". To some extend the altered voiding behaviour could be caused by an increased level of afferent activity of the lower urinary tract. The mechanisms remain largely unclear but are most likely caused by dysfunctions at both central and peripheral levels of control.

\section{Future perspectives}

In this thesis, through morphological, functional and behavioral studies, the structural and functional aspects of the sensory-motor interaction in the urinary bladder have been studied. Our studies identified the EP2 distribution in the guinea pig bladder wall. A hypothesis has been put forward suggesting the PG signal to be spread and augmented through interstitial cells as these cells possess the ability to produce PG through COXI as well as to respond to a PG signal through the EP2 receptor. The PG system is thought to play a role in the motor sensory system and more specific in the propagation of a signal expected the bladder wall. However, functional experiments are needed in order to confirm this hypothesis and the physiological significance of PG receptors in the bladder.

In a series of experiments, the different types of nerve fibres in the mouse bladder were studied through immunohistochemistry. The data show a variety of nerves in the bladder with a distinct 
local distribution. Further experiments breaching between functional and structural research is paramount to gain insight into the role of these different afferent nerve fibres during normal bladder functioning and in relation to functional bladder pathology, such as the overactive bladder syndrome.

non-voiding activity is seen in both normal and pathological conditions. In our experiments with anaesthetized guinea pigs we demonstrated the presence of non-voiding contractions during the filling phase and found the non-voiding contractions to progressively increase in amplitude and frequency as the bladder fills. The exact role of these non-voiding contractions is currently unknown, but they may be related to sensory function as they are only present when the bladder is connected to the spinal cord. Small non-voiding contractions are also present in humans as they have been shown in up to $70 \%$ of healthy volunteers during ambulatory urodynamics ${ }^{52}$. Further insight into the modulation of this specific pattern of small NVC may provide new diagnostic and therapeutic tools to assess and treat lower urinary tract dysfunctions.

With regard to changes in bladder morphology and behavior, we have conducted a series of experiments in transgenic mice. In our first study, we explored the differences in the structure of bladders from a transgenic mouse model of Alzheimer's disease with age matched control animals. From the data found, it is not possible to explain the origin or development of these differences. It is also difficult to explain fully, the physiological consequences. However, our data show that there are differences in the afferent system and also inevitably the motor-sensory system of the mouse model, which may lead to alterations in the local bladder control. These findings could aid in elucidating the underlying pathology of lower urinary tract dysfunction in humans with dementia and Alzheimer's disease and illustrate a co-involvement of the peripheral nervous system. Our results may also help to stimulate research on changes in the peripheral nervous system in animals models initially designed to study disease of the central nervous system. Structural immunohistochemical studies of bladder tissue of patients with Alzheimer's disease and physiological studies on the present mouse model are future possibilities to be investigated. The non-invasive method to evaluate voiding behaviour used in our study has to be combined with functional testing using cystometry to give more insight into the function and functional changes of the bladder itself in this animal model in the future. The chronology of the changes has to be studied by assessment of structure and function at different ages, which then, elucidate whether the changes are genetically determined or the consequence of further development. Moreover, a newly developed rat model for Alzheimer's disease could be an interesting model in terms of translational research to the human condition ${ }^{88}$. These models will be useful to elucidate whether functional changes develop first at the peripheral level or at the central level, i.e. due to changes in the bladder itself and/or in the brain in Alzheimer's disease. The exact underlying mechanism between Alzheimer disease and altered voiding behaviour is an important area to be explored in the future. 


\section{Conclusions}

Our morphological studies in the guinea pig bladder have shown EP2 receptor immunoreactivity located on the smooth muscle cells as well as on vimentin positive surface muscle and intramuscular interstitial cells. COXI is found to be expressed in the muscle interstitial cells. Furthermore, several different types of afferent nerve fibre in the bladder wall have been studied and at the bladder base dense immunoreactivity was seen to CGRP and VAChT on nerve fibres in the lamina propria compared to the lateral wall. Overall immunoreactivity to NF- and nNOS on nerve fibres was sparse in the lamina propria throughout the bladder. Functional studies have shown non-voiding contractions in all animals. These contractions increased in frequency and amplitude with the increase in filling rate and intravesical volume.

Our studies in the Alzheimer model mice revealed nerve fibres immunoreactive to VAChT in the inner and outer muscle bundles of APP ${ }^{S L} / \mathrm{PSI}^{\mathrm{MI} / 46 \mathrm{~L}}$ and control mice with no differences in the distribution. Within the lamina propria dense VAChT and nNOS immunoreactivity was seen in App-

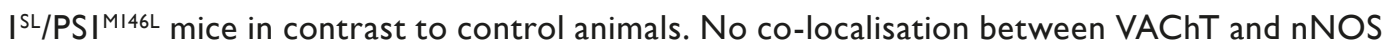
immunoreactive nerve fibres was present although we observed close proximity between them. Moreover, observational studies in the same mouse model showed anxiety-related behaviour and locomotion in an elevated zero maze task and open field task. The ApP ${ }^{\text {SL/PSIMI46L }}$ mice urinated more frequently in the centre zone compared to the control mice. However, in both groups the largest markings were found in the corner zones. The total volume of markings in the APP ${ }^{\text {SL/PSIMI46L }}$ mice was significantly lower. In both groups, the average volume of a marking in the corner zone was larger than in the centre zone. In the behavioural studies the APP ${ }^{\text {SL/PSIMI46L }}$ displayed more anxious behaviour. We concluded that APP ${ }^{\text {SL/PSIMI46L }}$ mice have a different voiding behaviour compared to control mice, i.e. urinating with small volumes and voiding in the centre of the cage. Additionally we saw increased anxiety-related behaviour.

Future research needs to extrapolate our data found in animal models to clinical studies revealing the pathophysiology of neurogenic OAB in Alzheimer's disease. We have show interesting insights revealing detailed sensory-motor neuronal networks in both mice and guinea pigs, discovered nonvoiding contractions and altered voiding behavior using various techniques. These new insights will lead to future research perspectives for $O A B$ and the aging population. 


\section{References}

I. Abrams, P., et al. The standardisation of terminology in lower urinary tract function: report from the standardisation sub-committee of the International Continence Society. Urology 61, 37-49 (2003).

2. Abrams, P., et al. Reviewing the ICS 2002 terminology report: the ongoing debate. Neurourology and urodynamics 28, 287 (2009).

3. Haylen, B.T., et al. An International Urogynecological Association (IUGA)/International Continence Society (ICS) joint report on the terminology for female pelvic floor dysfunction. Neurourology and urodynamics 29, 4-20 (2010).

4. Abrams, P., et al. Fourth International Consultation on Incontinence Recommendations of the International Scientific Committee: Evaluation and treatment of urinary incontinence, pelvic organ prolapse, and fecal incontinence. Neurourology and urodynamics 29, 213-240 (2010).

5. Cerruto, M.A., et al. Insight into new potential targets for the treatment of overactive bladder and detrusor overactivity. Urologia internationalis 89, I-8 (2012).

6. Milsom, I., et al. How widespread are the symptoms of an overactive bladder and how are they managed? A population-based prevalence study. BJU international 87, 760-766 (200I).

7. Cardozo, L. The overactive bladder syndrome: treating patients on an individual basis. BJU international 99 Suppl 3, 1-7 (2007).

8. Andersson, K.E. Drugs and future candidates. Can Urol Assoc J 5, SI3I-I33 (20II).

9. Mostwin, J.L., Karim, O.M., van Koeveringe, G. \& Brooks, E.L. The guinea pig as a model of gradual urethral obstruction. The Journal of urology 145, 854-858 (1991).

10. Klevmark, B. Motility of the urinary bladder in cats during filling at physiological rates. II. Effects of extrinsic bladder denervation on intramural tension and on intravesical pressure patterns. Acta physiologica Scandinavica I0I, I76-184 (1977).

II. Vaughan, C.W. \& Satchell, P.M. Role of sympathetic innervation in the feline continence process under natural filling conditions. Journal of neurophysiology 68, I842-I849 (1992).

12. Gillespie, J.I. A developing view of the origins of urgency: the importance of animal models. BJU international 96 Suppl I, 22-28 (2005).

13. Rahnama'i, M.S., et al. The relationship between prostaglandin E receptor I and cyclooxygenase I expression in guinea pig bladder interstitial cells: proposition of a signal propagation system. The Journal of urology 185, 315-322 (2011).

14. Rahnama'i, M.S., et al. Prostaglandin receptor EPI and EP2 site in guinea pig bladder urothelium and lamina propria. The Journal of urology 183, I24I-I247 (2010).

I5. de Jongh, R., et al. The localisation of cyclo-oxygenase immuno-reactivity (COXI-IR) to the urothelium and to interstitial cells in the bladder wall. Journal of cellular and molecular medicine (2008).

16. de Jongh, R., et al. The effects of exogenous prostaglandins and the identification of constitutive cyclooxygenase I and II immunoreactivity in the normal guinea pig bladder. BJU international I00, 419-429 (2007). 
Structural and Functional Aspects of Sensory-motor Interaction in the Urinary Bladder

17. Kohler, C., Ebert, U., Baumann, K. \& Schroder, H. Alzheimer's disease-like neuropathology of gene-targeted APP-SLxPSI mut mice expressing the amyloid precursor protein at endogenous levels. Neurobiology of disease 20, 528-540 (2005).

18. Rahnama'i, M.S., van Kerrebroeck, P.E., de Wachter, S.G. \& van Koeveringe, G.A. The role of prostanoids in urinary bladder physiology. Nature reviews. Urology 9, 283-290 (2012).

19. Maggi, C.A., et al. Prostanoids modulate reflex micturition by acting through capsaicin-sensitive afferents. Eur J Pharmacol 145, I05-II2 (1988).

20. McCafferty, G.P., Misajet, B.A., Laping, N.J., Edwards, R.M. \& Thorneloe, K.S. Enhanced bladder capacity and reduced prostaglandin E2-mediated bladder hyperactivity in EP3 receptor knockout mice. Am J Physiol Renal Physiol 295, F507-5I4 (2008).

21. Schussler, B. Comparison of the mode of action of prostaglandin E2 (PGE2) and sulprostone, a PGE2-derivative, on the lower urinary tract in healthy women. A urodynamic study. Urol Res 18, 349-352 (1990).

22. Ishizuka, O., Mattiasson, A. \& Andersson, K.E. Prostaglandin E2-induced bladder hyperactivity in normal, conscious rats: involvement of tachykinins? J Urol I53, 2034-2038 (1995).

23. Chuang, Y.C., et al. Intravesical botulinum toxin A administration inhibits COX-2 and EP4 expression and suppresses bladder hyperactivity in cyclophosphamide-induced cystitis in rats. Eur Urol 56, I59-166 (2009).

24. Su, X., et al. An excitatory role for peripheral EP3 receptors in bladder afferent function. Am J Physiol Renal Physiol 295, F585-594 (2008).

25. Ponglowhapan, S., Church, D.B. \& Khalid, M. Expression of prostaglandin E receptor subtypes in the canine lower urinary tract varies according to the gonadal status and gender. Theriogenology 74, I450-I466 (20I0).

26. Coleman, R.A., Smith, W.L. \& Narumiya, S. International Union of Pharmacology classification of prostanoid receptors: properties, distribution, and structure of the receptors and their subtypes. Pharmacol Rev 46, 205-229 (1994).

27. Ikeda, M., Kawatani, M., Maruyama, T. \& Ishihama, H. Prostaglandin facilitates afferent nerve activity via EPI receptors during urinary bladder inflammation in rats. Biomedical research (Tokyo, Japan) 27, 49-54 (2006).

28. Maggi, C.A., Lecci, A., Santicioli, P., Del Bianco, E. \& Giuliani, S. Cyclophosphamide-induced cystitis in rats: involvement of capsaicin-sensitive primary afferents. Agents Actions $38 \mathrm{Spec}$ No, C28-30 (1993).

29. Yu, W., Hill, W.G., Apodaca, G. \& Zeidel, M.L. Expression and distribution of transient receptor potential (TRP) channels in bladder epithelium. Am J Physiol Renal Physiol 300, F49-59.

30. Birder, L.A., et al. Vanilloid receptor expression suggests a sensory role for urinary bladder epithelial cells. Proc Natl Acad Sci U S A 98, I3396-I340I (200I).

31. Gillespie, J.I. Inhibitory actions of calcitonin gene-related peptide and capsaicin: evidence for local axonal reflexes in the bladder wall. BJU Int 95, I49-I56 (2005). 
32. Daly, D., Rong, W., Chess-Williams, R., Chapple, C. \& Grundy, D. Bladder afferent sensitivity in wild-type and TRPVI knockout mice. J Physiol 583, 663-674 (2007).

33. Lawson, S.N., Perry, M.J., Prabhakar, E. \& McCarthy, P.W. Primary sensory neurones: neurofilament, neuropeptides, and conduction velocity. Brain Res Bull 30, 239-243 (1993).

34. Yoshimura, N., Erdman, S.L., Snider, M.W. \& de Groat, W.C. Effects of spinal cord injury on neurofilament immunoreactivity and capsaicin sensitivity in rat dorsal root ganglion neurons innervating the urinary bladder. Neuroscience 83, 633-643 (1998).

35. Janig, W. \& Morrison, J.F. Functional properties of spinal visceral afferents supplying abdominal and pelvic organs, with special emphasis on visceral nociception. Progress in brain research 67, 87-II4 (1986).

36. Gillespie, J.I., Markerink-van Ittersum, M. \& de Vente, J. Sensory collaterals, intramural ganglia and motor nerves in the guinea-pig bladder: evidence for intramural neural circuits. Cell and tissue research 325, 33-45 (2006).

37. Gosling, J.A. \& Dixon, J.S. Sensory nerves in the mammalian urinary tract. An evaluation using light and electron microscopy. J Anat II7, I33-I44 (1974).

38. Sann, H., McCarthy, P.W., Mader, M. \& Schemann, M. Choline acetyltransferase-like immunoreactivity in small diameter neurones of the rat dorsal root ganglion. Neurosci Lett 198, 17-20 (1995).

39. Mohammed, H.A. \& Santer, R.M. Distribution and changes with age of calcitonin gene-related peptide- and substance P-immunoreactive nerves of the rat urinary bladder and lumbosacral sensory neurons. Eur J Morphol 40, 293-30I (2002).

40. Dixon, J.S., Jen, P.Y. \& Gosling, J.A. The distribution of vesicular acetylcholine transporter in the human male genitourinary organs and its co-localization with neuropeptide $Y$ and nitric oxide synthase. Neurourol Urodyn 19, 185-194 (2000).

4I. Nandigama, R., et al. Muscarinic acetylcholine receptor subtypes expressed by mouse bladder afferent neurons. Neuroscience 168, 842-850.

42. Andersson, K.E. \& Hedlund, P. Pharmacologic perspective on the physiology of the lower urinary tract. Urology 60, I3-20; discussion 20-II (2002).

43. Andersson, K.E. \& Wein, A.J. Pharmacology of the lower urinary tract: basis for current and future treatments of urinary incontinence. Pharmacol Rev 56, 58I-63I (2004).

44. Andersson, K.E. \& Persson, K. Nitric oxide synthase and the lower urinary tract: possible implications for physiology and pathophysiology. Scand J Urol Nephrol Suppl 175, 43-53 (1995).

45. Zhou, Y. \& Ling, E.A. Colocalization of nitric oxide synthase and some neurotransmitters in the intramural ganglia of the guinea pig urinary bladder. J Comp Neurol 394, 496-505 (1998).

46. Gillespie, J.I. \& Drake, M.J. The actions of sodium nitroprusside and the phosphodiesterase inhibitor dipyridamole on phasic activity in the isolated guinea-pig bladder. BJU Int 93, 85I-858 (2004).

47. Smet, P.J., Jonavicius, J., Marshall, V.R. \& de Vente, J. Distribution of nitric oxide synthase- 
Structural and Functional Aspects of Sensory-motor Interaction in the Urinary Bladder

immunoreactive nerves and identification of the cellular targets of nitric oxide in guinea-pig and human urinary bladder by cGMP immunohistochemistry. Neuroscience 7I, 337-348 (1996).

48. Gillespie, J.I. Noradernaline inhibits autonoumous activity in the isolated guinea pig bladder BJU international feb, 40I-409 (2004).

49. Hardwick, J.C., Mawe, G.M. \& Parsons, R.L. Evidence for afferent fiber innervation of parasympathetic neurons of the guinea-pig cardiac ganglion. J Auton Nerv Syst 53, I66- 174 (1995).

50. Gillespie, J.I., Harvey, I.J. \& Drake, M.J. Agonist- and nerve-induced phasic activity in the isolated whole bladder of the guinea pig: evidence for two types of bladder activity. Exp Physiol 88, 343357 (2003).

51. Kanai, A., et al. Origin of spontaneous activity in neonatal and adult rat bladders and its enhancement by stretch and muscarinic agonists. Am J Physiol Renal Physiol 292, FI065-I072 (2007).

52. van Waalwijk van Doorn, E.S., Remmers, A. \& Janknegt, R.A. Conventional and extramural ambulatory urodynamic testing of the lower urinary tract in female volunteers. The Journal of urology 147, I319-1325; discussion I326 (1992).

53. Robertson, A.S. Behaviour of the human bladder during natural filling: the Newcastle experience of ambulatory monitoring and conventional artificial filling cystometry. Scandinavian journal of urology and nephrology. Supplementum 20I, 19-24 (1999).

54. Drake, M.J., Harvey, I.J. \& Gillespie, J.I. Autonomous activity in the isolated guinea pig bladder. Experimental physiology 88, 19-30 (2003).

55. Streng, T., Hedlund, P., Talo, A., Andersson, K.E. \& Gillespie, J.I. Phasic non-micturition contractions in the bladder of the anaesthetized and awake rat. BJU international 97, I094-II0 I (2006).

56. Yu, Y. \& de Groat, W.C. Sensitization of pelvic afferent nerves in the in vitro rat urinary bladder-pelvic nerve preparation by purinergic agonists and cyclophosphamide pretreatment. American journal of physiology 294, FII46-II56 (2008).

57. Coolsaet, B.L., Van Duyl, W.A., Van Os-Bossagh, P. \& De Bakker, H.V. New concepts in relation to urge and detrusor activity. Neurourology and urodynamics 12, 463-47I (1993).

58. McCarthy, C.J., et al. Spontaneous contractions evoke afferent nerve firing in mouse bladders with detrusor overactivity. The Journal of urology I8I, I459-I466 (2009).

59. Habler, H.J., Janig, W. \& Koltzenburg, M. Myelinated primary afferents of the sacral spinal cord responding to slow filling and distension of the cat urinary bladder. The Journal of physiology 463 , 449-460 (1993).

60. Shea, V.K., Cai, R., Crepps, B., Mason, J.L. \& Perl, E.R. Sensory fibres of the pelvic nerve innervating the Rat's urinary bladder. Journal of neurophysiology 84, 1924-1933 (2000).

6I. De Wachter, S., De Laet, K. \& Wyndaele, J.J. Does the cystometric filling rate affect the afferent bladder response pattern? A study on single fibre pelvic nerve afferents in the rat urinary bladder. Neurourology and urodynamics 25, I62-167 (2006). 
62. Moss, N.G., Harrington, W.W. \& Tucker, M.S. Pressure, volume, and chemosensitivity in afferent innervation of urinary bladder in rats. The American journal of physiology 272, R695-703 (1997).

63. Zagorodnyuk, V.P., Costa, M. \& Brookes, S.J. Major classes of sensory neurons to the urinary bladder. Autonomic neuroscience : basic \& clinical 126-127, 390-397 (2006).

64. Morrison, J. The activation of bladder wall afferent nerves. Experimental physiology 84, I3I-I36 (1999).

65. Iggo, A. Tension receptors in the stomach and the urinary bladder. The Journal of physiology I28, 593-607 (1955).

66. De Laet, K., De Wachter, S. \& Wyndaele, J.J. Systemic oxybutynin decreases afferent activity of the pelvic nerve of the rat: new insights into the working mechanism of antimuscarinics. Neurourol Urodyn 25, I56-16I (2006).

67. Wyndaele, J.J. \& De Wachter, S. The sensory bladder (I): an update on the different sensations described in the lower urinary tract and the physiological mechanisms behind them. Neurourol Urodyn 27, 274-278 (2008).

68. Gillespie, J.I., van Koeveringe, G.A., de Wachter, S.G. \& de Vente, J. On the origins of the sensory output from the bladder: the concept of afferent noise. BJU international (2009).

69. Ransmayr, G.N., et al. Lower urinary tract symptoms in dementia with Lewy bodies, Parkinson disease, and Alzheimer disease. Neurology 70, 299-303 (2008).

70. Silva, C., et al. Bladder sensory desensitization decreases urinary urgency. BMC urology 7, 9 (2007).

7I. Liu, L., et al. The molecular basis of urgency: regional difference of vanilloid receptor expression in the human urinary bladder. Neurourol Urodyn 26, 433-438; discussion 439; discussion 45I-433 (2007).

72. Smet, P.J., Moore, K.H. \& Jonavicius, J. Distribution and colocalization of calcitonin gene-related peptide, tachykinins, and vasoactive intestinal peptide in normal and idiopathic unstable human urinary bladder. Laboratory investigation; a journal of technical methods and pathology 77, 37-49 (1997).

73. Smet, P.J., Edyvane, K.A., Jonavicius, J. \& Marshall, V.R. Neuropeptides and neurotransmittersynthesizing enzymes in intrinsic neurons of the human urinary bladder. J Neurocytol 25, II2-124 (1996).

74. Gabella, G. \& Davis, C. Distribution of afferent axons in the bladder of rats. J Neurocytol 27, I4I-I55 (1998).

75. Dixon, J.S., Jen, P.Y. \& Gosling, J.A. A double-label immunohistochemical study of intramural ganglia from the human male urinary bladder neck. J Anat 190 ( Pt I), I25-134 (1997).

76. Pirker, M.E., Montedonico, S., Rolle, U., Austvoll, H. \& Puri, P. Regional differences in nitrergic neuronal density in the developing porcine urinary bladder. Pediatr Surg Int 2I, I6I-I68 (2005).

77. Gillespie, J.I. The autonomous bladder: a view of the origin of bladder overactivity and sensory urge. BJU international 93, 478-483 (2004). 
Structural and Functional Aspects of Sensory-motor Interaction in the Urinary Bladder

78. Williams, M.E. \& Pannill, F.C., 3rd. Urinary incontinence in the elderly: physiology, pathophysiology, diagnosis, and treatment. Ann Intern Med 97, 895-907 (1982).

79. Lee, S.H., Cho, S.T., Na, H.R., Ko, S.B. \& Park, M.H. Urinary incontinence in patients with Alzheimer's disease: relationship between symptom status and urodynamic diagnoses. International journal of urology : official journal of the Japanese Urological Association 21, 683-687 (2014).

80. Gevaert, T., et al. Deletion of the transient receptor potential cation channel TRPV4 impairs murine bladder voiding. The Journal of clinical investigation II7, 3453-3462 (2007).

8I. Serra, L., et al. Relationship between cognitive impairment and behavioural disturbances in Alzheimer's disease patients. Behav Neurol 23, 123-130.

82. Vanmierlo, T., et al. Liver $X$ receptor activation restores memory in aged AD mice without reducing amyloid. Neurobiology of aging (2009).

83. Fan, Y.H., Lin, A.T., Wu, H.M., Hong, C.J. \& Chen, K.K. Psychological profile of female patients with dysfunctional voiding. Urology 7I, 625-629 (2008).

84. Klausner, A.P., et al. Does oxybutynin alter plaques, amyloid beta peptides and behavior in a mouse model of Alzheimer's disease? J Urol I79, II73-II77 (2008).

85. Fowler, C.J., Griffiths, D. \& de Groat, W.C. The neural control of micturition. Nature reviews. Neuroscience 9, 453-466 (2008).

86. Biallosterski, B.T., et al. Changes in bladder innervation in a mouse model of Alzheimer's disease. Journal of chemical neuroanatomy 39, 204-210.

87. Han, D. \& Wang, Y. Urinary Incontinence in Dementia Incont Pelvic Floor Dysfunct 2, 63-66 (2008).

88. Cohen, R.M., et al. A transgenic Alzheimer rat with plaques, tau pathology, behavioral impairment, oligomeric abeta, and frank neuronal loss. J Neurosci 33, 6245-6256. 




\section{Vallorisation Paragraph}

\section{The clinical problem}

The overactive bladder syndrome (OAB) is defined by the International Continence Society (ICS) as urinary urgency which is accompanied by urinary frequency and nocturia, with or without urgency urinary incontinence ${ }^{1,2}$. $O A B$ is a significant problem in terms of quality of life and costs, especially in aging societies as in the Netherlands. The aetiology and underlying pathophysiology is heterogeneous and not well understood. The mainstay of current therapy, is antimuscarinic drugs which have limited efficiency and poor compliance due to side effects. Therefore there is need for better therapeutic modalities for patients with $O A B$ symptoms. About only half of $O A B$ patients have urodynamically proven detrusor overactivity (overactive contractions of the bladder). $O A B$ has a greater impact on people's quality of life than diabetes ${ }^{2-4}$ and an economic burden and cost comparable to rheumatoid arthritis and asthma ${ }^{5}$. Therefore, $O A B$ deserves more research resources and research efforts. Those affected by the symptoms of $O A B$ tend to curtail their participation in social activities and isolate themselves and are predisposed to depression ${ }^{6}$. Furthermore, there is probably an underestimation of the prevalence due to embarrassment ${ }^{7-10}$.

\section{Prevalence}

$O A B$ affects nearly 100 million people in the Western world ( 33 million in the US and 66 million in the European Union $)^{11,12}$ and has severe effects on quality of life and ability to work. OAB has an incidence of, up to $17 \%$ in the Western population" and an overall prevalence of $16.6 \%$ in Europe ${ }^{12}$. The prevalence of $O A B$ in the United States is estimated 26 to $33 \%$ in men and from 27 to $46 \%$ in women ${ }^{13}$.

\section{Socio-economic burden}

The total economic cost of OAB is high. In 2002 the costs in the US were approximately $\$ 12.7$ billion which increased to $€ 22$ billion/year in 2005. Less than $3 \%$ of the patients regain long lasting continence. Therefore, the above mentioned costs are likely to be an underestimation and most probably, the problem is much larger ${ }^{10,12-14}$. The exact economic costs and prevalence of $O A B$ in the Netherlands are unknown. However, it has been calculated that about $€ 200$ million are spent annually on protective material such as incontinence pads. In Germany, the direct annual costs have been estimated to be the same as costs of other chronic diseases such as dementia or, diabetes mellitus ${ }^{15}$. Other studies have compared the major costs of $O A B$ to rheumatoid arthritis and asthma ${ }^{5}$. From those who suffer from $O A B$, only $28 \%$ sought help and only half of those, currently receive treatment. Less than $3 \%$ of the patients regain long lasting continence. Therefore, the above mentioned costs are likely to be an underestimation and most probably, the problem is much larger ${ }^{10,12-14}$. As the incidence of $O A B$ 
Structural and Functional Aspects of Sensory-motor Interaction in the Urinary Bladder

increases with age, it will become an even more important problem in the coming years in our aging society. Hence, $O A B$ is a major problem affecting a large number of individuals and there is an urgent need for new insights into the problem and innovative therapeutic modalities.

\section{Current therapies for $O A B$}

Since OAB symptoms have been shown to be associated with detrusor overactivity, it was argued that drugs affecting contractility would alleviate symptoms ${ }^{16}$. Activity in the bladder smooth muscle is initiated by muscarinic receptor stimulation. The current treatment mainly consists of antimuscarinic agents, which have a slightly better effect than placebo, but poor patient compliance, due to their side effects and the lack of sufficient efficacy ${ }^{10,16}$. Therefore, it is desirable that alternative treatment methods are developed and made available for patients.

\section{Knowledge exchange and impact}

The results of our study can be beneficial for many. First place the patients suffering from OAB who can benefit from better treatment. As there is a strong correlation between $O A B$ and/or incontinence on one side, and depression on the other side, not only the OAB problem could be tackled, but also depression in these patients can eventually be treated more effectively. Furthermore, there is a potential benefit in the overall aging problem. As $O A B$ increases with aging and as we require selfreliance from elderly people, better $O A B$ treatment will allow them to participate more widely in the society. Another potential beneficial effect is the cost reduction for insurance companies that currently have to pay for incontinence pads and for ineffective treatment modalities for years. In addition care costs for an aging population increases dramatically due to urinary incontinence. The cost reduction will indirectly be beneficial for the society as it will reduce health costs. Moreover, there will be potentially less sick leave, which will again reduce costs for the society. In addition, if $O A B$ is treated more effectively, less incontinence material will be used and disposed which besides the cost reduction will be beneficial for the environment. The results of our study are relevant and interesting for researchers and science in general. Our results can further be used to design specific research in related disorders. A better understanding of bladder physiology and pathophysiology of $\mathrm{OAB}$ could be helpful in research in the field of detrusor underactivity, for which currently there is no treatment available. Bladder pain syndrome is another example of a condition that can benefit from the results presented in this thesis. Patients affected experience tremendous negative effects on their quality of life due to symptoms of $O A B$ with the addition of major invalidating pain symptoms. Moreover, a large group of patients with partial or complete spinal cord lesions suffer from bladder impairment as well as patients with dementia and Alzheimer's disease. All these conditions could benefit from results of our study. A better categorization of $O A B$ patients could also be helpful for clinicians writing or using clinical guidelines for treatment of voiding disorders such as $O A B$. 


\section{References}

I. Abrams, P., et al. The standardisation of terminology of lower urinary tract function: report from the Standardisation Sub-committee of the International Continence Society. American journal of obstetrics and gynecology 187, 116-126 (2002).

2. Komaroff, A.L., et al. Health status in patients with chronic fatigue syndrome and in general population and disease comparison groups. The American journal of medicine I0I, 28I-290 (1996).

3. Liberman, J.N., et al. Health-related quality of life among adults with symptoms of overactive bladder: results from a U.S. community-based survey. Urology 57, 1044-1050 (200I).

4. Hashim, H. \& Abrams, P. Overactive bladder: an update. Current opinion in urology 17, 231-236 (2007).

5. Coyne, K.S., et al. Impact of overactive bladder on work productivity. Urology 80, 97-103 (20I2).

6. Ouslander, J.G. Management of overactive bladder. N EnglJ Med 350, 786-799 (2004).

7. Abrams, P., Kelleher, C.J., Kerr, L.A. \& Rogers, R.G. Overactive bladder significantly affects quality of life. The American journal of managed care 6, S580-590 (2000).

8. Chiaffarino, F., Parazzini, F., Lavezzari, M., Giambanco, V. \& Gruppo Interdisciplinare di Studio Incontinenza, U. Impact of urinary incontinence and overactive bladder on quality of life. European urology 43, 535-538 (2003).

9. Goepel, M., Hoffmann, J.A., Piro, M., Rubben, H. \& Michel, M.C. Prevalence and physician awareness of symptoms of urinary bladder dysfunction. European urology 4I, 234-239 (2002).

10. Cardozo, L. The overactive bladder syndrome: treating patients on an individual basis. BJU international 99 Suppl 3, I-7 (2007).

II. Hu, T.W., et al. Costs of urinary incontinence and overactive bladder in the United States: a comparative study. Urology 63, 46I-465 (2004).

12. Milsom, l., et al. How widespread are the symptoms of an overactive bladder and how are they managed? A population-based prevalence study. BJU international 87, 760-766 (200I).

13. Coyne, K.S., Margolis, M.K., Kopp, Z.S. \& Kaplan, S.A. Racial differences in the prevalence of overactive bladder in the United States from the epidemiology of LUTS (EpiLUTS) study. Urology 79, 95-I0I (2012).

14. Van Kerrebroeck, P.E. A treatment algorithm for the overactive bladder. BJU international 83 Suppl 2, 29-30 (1999).

15. Klotz, T., Bruggenjurgen, B., Burkart, M. \& Resch, A. The economic costs of overactive bladder in Germany. European urology 5I, 1654-1662; discussion 1662-1653 (2007).

16. Andersson, K.E. Drugs and future candidates. Can Urol Assoc J 5, SI3I-I33 (20II). 



\section{Summary}

The general aim of this thesis was twofold. We wanted to further investigate the motor sensory system of the bladder at a structural and functional level. To this end, we first conducted morphological studies in guinea pigs and mice, functionally we looked at non-voiding contractions in guinea pigs. Secondly we investigated a model for bladder dysfunction in Alzheimer's disease. Several experiments have been conducted with these aims as described in the different chapters. The experimental data presented in this thesis can be summarized as follows:

In Chapter 2 we investigated the distribution of prostaglandin E receptor type 2 (EP2) in the bladder muscle layers and its spatial relationship to cyclo-oxygenase type I (COXI). Therefore the bladders of 12 male guinea pigs were stained with antibodies to EP2, COX I and vimentin. Our results show EP2 receptor immunoreactivity located on the smooth muscle cells as well as on vimentin positive surface muscle and intramuscular interstitial cells. COXI is expressed in the muscle interstitial cells. Double staining with EP2 and COX I suggests that the regions of a cell expressing EP2 are different from those expressing COXI. This data suggests a role of this network in the propagation of signals. A rise in PG levels may have a modulatory role on the non-voiding bladder contractions by changing the threshold level for excitability of the interstitial cell network.

In chapter 3 we explored and identified sub-types of afferent nerve fibres in the mouse bladder wall, based on morphological criteria and analyse regional differences. 27 bladders were stained for Protein Gene Product 9.5 (PGP), anti-calcitonin gene related polypeptide (CGRP), antiNeurofilament (NF), anti-vesicular acetylcholine transporter (VAChT) and anti-neuronal nitric oxide synthase (nNOS). Our results show several different types of afferent nerve fibre as well as a spacial distribution in the bladder wall. At the bladder base dense immunoreactivity was seen to CGRP and VAChT on nerve fibres in the lamina propria compared to the lateral wall. Overall immunoreactivity to NF- and $\mathrm{nNOS}$ on nerve fibres was sparse in the lamina propria throughout the bladder. At the lateral wall the inner muscle nerve fibres densely stained to CGRP. NF, VAChT and $\mathrm{nNOS}$ staining nerve fibres were evenly distributed in the different muscle layers throughout the bladder. Nerve fibre terminals expressing CGRP and NF were found within the extra-mural ganglia at the bladder base. The identification of these different staining patterns as well as a different anatomical distribution suggests a functional heterogeneity of afferent nerve fibres in the urinary bladder.

In chapter 4 we focused on non-voiding contractions (NVCs) in the guinea pig bladder. Fourteen guinea pigs were anesthetized and the bladder catheterised at the dome. The group was divided, in one group NVCs were recorded when the bladders were continuously filled at two different physiological rates, in the other group isovolumetric cystometry was performed filling the bladder with increments, recording at low, medium and high intravesical volume. The results show that NVCs were apparent in all animals. Secondly, we saw that contractions increased in frequency 
and amplitude with the increase in filling rate and intravesical volume. The small and large NVCs differed in frequency and occurred at different periods of bladder filling. This may illustrate different afferents functioning during bladder filling.

In chapter 5 we studied the morphological structure of bladders from a transgenic mouse model of Alzheimer's disease. Transgenic mice (APP ${ }^{\text {SL/PSIMI46L) }}$ aged 24 months were used and compared with age matched control animals of $\mathrm{C} 57$ black mice. The bladders from each group were removed and stained for vesicular acetylcholine transporter (VAChT) and neuronal nitric oxide synthase (nNOS). Nerve fibres immunoreactive to VAChT were observed in the inner and outer muscle bundles of $A P P^{S L} / P S I^{M I 46 L}$ and control mice with no differences in the distribution. Within the lamina propria dense VAChT and nNOS immunoreactivity was seen in App I ${ }^{\mathrm{SL}} / \mathrm{PS} \mathrm{I}^{\mathrm{M} / 46 \mathrm{~L}}$ mice in contrast to control animals. No co-localisation between VAChT and nNOS immunoreactive nerve fibres was present although we observed close proximity between them. An unusual feature of the APP ${ }^{\text {SL/PSIMI46L }}$ mouse bladder was the presence of nerve cell bodies, which were seen in all bladders. They were found as single or as ganglion-like groups of cells and were located in all layers of the bladder wall. No nerve cells or small ganglia were noted in any of the control bladders. These structural differences in the bladders of APP ${ }^{\text {SL }} / \mathrm{PSI}^{\mathrm{MI}}{ }^{46 \mathrm{~L}}$ mice may indicate a changed generation and modulation of sensation. In chapter 6 we again used the transgenic mouse $A_{P P}{ }^{S L} / P S I^{M I 46 L}$ to examine the urinary marking behaviour in relation to affective behavior in this model. In total 7 mice aged 18 months were compared to 9 aged matched controls. Voiding behaviour was studied by a modified filter paper assay in combination with video tracing whereby the cage was divided into one centre zone and two corner zones. Anxiety-related behaviour and locomotion were tested in an elevated zero maze task and open field task. Our results show that the APP ${ }^{S L} / P S I^{M I 46 L}$ mice urinated more frequently in the centre zone compared to the control mice. However, in both groups the largest markings were found in the corner zones. The total volume of markings in the APP ${ }^{\mathrm{SL}} / \mathrm{PS} \mathrm{I}^{\mathrm{MI}}{ }^{46 \mathrm{~L}}$ mice was significantly lower. In both groups, the average volume of a marking in the corner zone was larger than in the centre zone. In the behavioural studies the APP ${ }^{S L} / P S I^{M / 46 L}$ displayed more anxious behaviour. We concluded that APP ${ }^{S L} / P S I^{M 146 L}$ mice have a different voiding behaviour compared to control mice, e.g. urinating with small volumes and voiding in the centre of the cage. Additionally we saw increased anxiety-related behaviour. These observations in the change in voiding pattern of the APP ${ }^{\text {SL/PSIMI46L }}$ mouse could indicate a change in afferent activity of the lower urinary tract. 




\title{
Samenvatting
}

\author{
(Summary in Dutch)
}

In dit proefschrift zijn functionele en structurele studies naar het motor-sensorische system van de urineblaas beschreven. In het eerste deel betreft structurele studies. Op functioneel niveau hebben we in cavia's en muizen gekeken naar blaas contracties die niet tot mictie leiden in cavia's. In het tweede deel is gebruik gemaakt van een model voor blaasdysfunctie bij de ziekte van Alzheimer. De in het proefschrift beschreven experimentele data kunnen als volgt worden samengevat.

$\mathrm{Na}$ een inleiding in hoofdstuk I wordt in hoofdstuk 2, de distributie van de prostaglandine E receptor type 2 (EP2) in spierlagen van de blaas beschreven, alsmede de relatie van EP2 tot de distributie van cyclo-oxygenase type I enzym (COXI). De resultaten tonen immunoreactiviteit aan tegen EP2 bij de gladde spiercellen en bovendien op de vimentine-positieve interstitiele cellen van zowel de oppervlakte spier als die tussen de spier liggen. COX I is zichtbaar in interstitiele cellen in de blaasspier. De expressie plaats van EP2 op de cel is anders dan die van COXI.

In hoofdstuk 3 zijn de resultaten van een studie naar verschillende sub-types van afferente zenuwen in muizen weergegeven. De zenuwen zijn te identificeren op basis van morfologische criteria en de regio van de blaas waar de zenuw vezels zich bevinden. Er zijn verschillende types van afferente zenuw vezels op verschillende locaties in de blaaswand gevonden. De zenuwvezels in de blaasbodem, tonen een sterke immuunreactiviteit voor CGRP en VAChT in de lamina propria. Er was over het algemeen weinig kleuring van NF- and nNOS op zenuwvezels in de lamina propria door de hele blaas. De zenuwvezels ter hoogte van de laterale wand bij de binnenste spierlaag kleuren sterk voor CGRP. Zenuwvezels die voor NF, VAChT en nNOS kleuren zijn gelijk verdeeld over de verschillende spierlagen in de blaas. Ter hoogte van de blaas bodem buiten de blaaswand waren ganglia zichtbaar met CGRP en NF positieve zenuw uitlopers. Het patroon van aankleuring en localisatie van de zenuwvezels in de blaaswand kan een aanwijzing zijn voor het bestaan van verschillend functionerende afferente zenuw vezels in the urineblaas.

De resultaten van een studie naar de non-voiding contracties (NVCs) in de caviablaas zijn gepresenteerd in hoofdstuk 4. Deze studie toont NVCs in alle bestudeerde dieren. Bovendien is er een toenemende frequentie en sterkte van de non-voiding contracties bij enerzijds een groter blaasvolume, en anderzijds een snellere vullingssnelheid. Verder lijkt er een verschil te zijn tussen de kleine en grote blaas contracties betreffende frequentie en fase van blaasvulling (begin verus einde van de vulling).

Een studie naar de morfologie van de blaas van een transgeen muismodel voor de ziekte van Alzheimer is gepresenteerd in hoofdstuk 5. VACht positieve zenuwvezels zijn zichtbaar in zowel de binnenste als buitense spierlaag in APP ${ }^{\text {SL/PSI }}{ }^{\text {MI46L }}$ en in controle muizen zonder verschil tussen de groepen. VAChT en nNOS immunoreactiviteit werd alleen gezien in de lamina propria van 
Structural and Functional Aspects of Sensory-motor Interaction in the Urinary Bladder

AppI $\left.\right|^{\text {SL/PSI }}{ }^{\text {MI46L }}$ muizen. Alhoewel de zenuw vezels dicht bij elkaar komen zijn er geen aanwijzingen

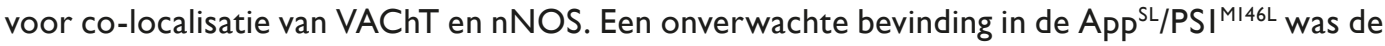
aanwezigheid van cellichamen midden in de blaaswand. Deze observatie was in alle dieren consistent. De cellichamen bestonden uit of wel enkele cellen dan wel ganglion-achtige structuren en werden waargenomen in alle lagen door de blaaswand heen. In de controle blazen warden geen cel lichamen in de blaaswand waargenomen.

In hoofdstuk 6 is een andere studie met de transgene muis APP ${ }^{S L} / P S I^{M I 46 L}$ weergegeven. Dit observationeel onderzoek naar plasgedrag in relatie tot affectief gedrag van de muizen laat zien dat de APP ${ }^{\text {SL/PSIMI46L }}$ muizen vaker plassen in de centrum zones in vergelijking met controle muizen. De grootste markings van beide groepen werden gevonden in de hoekzones. Het totaal geplaste volumes van de APP ${ }^{\text {SL }}$ PSI ${ }^{\text {MI }}{ }^{\text {LL }}$ muizen was significant lager. In beide groepen is het gemiddelde volume van een marking in de hoekzone groter dan in de centrumzone. Uit de gedragsexperimenten blijkt dat de ApP ${ }^{\text {SL/PSI }}{ }^{\mathrm{M} / 46 \mathrm{~L}}$ veel angstiger zijn.

Hoofdstuk 7 betreft een algemene discussie van alle resultaten van de beschreven experimenten. 



\section{Acknowledgments - Dankwoord}

Het dankwoord, een plek in je boekje voor bezinning. Het voelt wat onwennig, maar langzaam aan begin je te geloven dat het eind inderdaad in zicht is...

Luctor et emergo; zo voelt het wel. Mijn pad was oneffen, soms mistig, steil en ineens helder, open en recht. Mijn promotie is tot stand gekomen met steun, advies en motivatie van velen. Bij deze wil graag mijn dank uiten aan degene die hebben bijgedragen. Allereerst mijn promotie team.

Prof. Van Kerrebroeck, mijn eerste promotor, dank u voor de mogelijkheid om bij u promotie onderzoek te kunnen doen. Ik heb de afgelopen jaren veel van u geleerd. Met name veel dank voor uw hulp bij het afronden.

Prof. de Wachter, mijn tweede promotor, beste Stefan, je bent op belangrijke momenten van grote waarde en steun geweest tijdens mijn onderzoeksperiode. Dank voor je technisch inzicht bij de experimenten en het begeleiden van het schrijven van de Manuschripten.

Dr. van Koeveringe, mijn eerste co-promotor, beste Gommert, ik zie jouw als de vuurtoren in mijn promotie onderzoek. Als ik niet wist waar ik heen moest, was jij er als vast aanspreekpunt, motivator, coach. Ik ben je door de jaren heen heel erg gaan waarderen. Ik bewonder je doorzettingsvermogen en rust en enthousiasme. Ik heb van jou geleerd wat het is om onderzoek te doen, met alles wat daarbij komt kijken. Ik zie mijn promotie dan ook als ons succes. Ik hoop dat jij er ook trots op bent!

Dr. Rahnama'i, mijn tweede co-promotor, beste Sajjad, leuk om jou als mijn co-promotor te hebben. Je mag er trots op zijn. Je bent ontzettend belangrijk geweest bij de afronding van mijn promotie. We zijn natuurlijk begonnen als collega promovendi in Maastricht en hebben het grootste deel van onze onderzoekstijd samen opgetrokken. Ik heb genoten van de humor en gezelligheid en bewonder je gedrevenheid voor resultaat. Je bent erg succesvol in je academische carrière tot nu toe, met mijn promotie als een volgend hoogtepunt. Wie had dat ooit gedacht?

Verder zijn in mijn onderzoeksperiode diverse mensen van het Neuroscience laboratorium belangrijk geweest.

Prof. Steinbusch, achter de schermen bent u van grote waarde geweest tijdens mijn onderzoeksperiode en nu natuurlijk ook met de afronding van mijn promotie. Ik wil u voor alles heel erg bedanken. Zonder u was het boekje er niet geweest. 
Structural and Functional Aspects of Sensory-motor Interaction in the Urinary Bladder

Prof. Gillespie, dear James, during my research period in Maastricht, you have been invaluable because of your immunohistochemical knowledge. I have been lucky to learn this type of research from you in Maastricht and Newcastle. Thank you for your contribution to my thesis.

Dr. de Vente, beste Jan, voor mij ben jij DE wetenschapper. Kijken, controleren, herhalen, discussieren. Je subtiele en rustige manier van werken zijn voor mij kenmerkend. Ik heb je aanwijzingen in het begin van mijn promotie traject ontzettend gewaardeerd.

Graag wil ik ook de laboratorium technici van de afdeling Neuroscience bedanken voor alle hulp bij de experimenten, met name Hellen en Denise. Beste Hellen, dank voor alle uitleg bij de immunohistochemische kleuringen, de microscoop en nog veel meer.

Verder wil ik alle mede AIO's van de afdeling Neuroscience tijdens mijn promotie tijd bedanken voor een leuke, leerzame, gezellige en mooie periode Annerieke, Sven, Jochen, Eva B, Eva van D, Marijke, etc. En specifiek de neurochirurgie groep, Sonny, Mark, Antony, Tibault, Ali dank / thank you all.

Mijn collega's van de urologie wil ik ook graag bedanken voor alle steun en gezelligheid tijdens mijn onderzoekstijd in Maastricht. Randall, Tom, Rhea, Simone en Paul naast Sajjad waren jullie met wie ik het meest heb gewerkt. Dank!

Verder wil ik de leden van de leescommissie bedanken voor de beoordeling van mijn promotie.

Behalve deze promotie heeft mijn avontuur in Maastricht mij ook geleid naar de liefde. Lieve Rinske wat ben ik toch gelukkig met jou. Ik geniet van je kracht, nieuwsgierigheid, creativiteit en lef. Ik ben zo blij met onze baba's. Ik vind het heerlijk om het leven met jou te kunnen delen. Kus. 




\section{Curriculum Vitae}

Bart Biallosterski werd op 16 april 1979 geboren in Haarlem. In 1998 haalde hij zijn VWO diploma op de Kennemer Lyceum te Overveen. Aansluitend studeerde hij 2 jaar Fysiologie in Schotland aan de Universiteit van Dundee, waarna hij in 2000 begon met de studie Geneeskunde aan de Universiteit van Groningen. In 2006 ontving hij zijn artsenbul. Hierna werkte hij 10 maanden als ANIOS Heelkunde in het Ziekenhuis Gooi Noord te Blaricum (heden; Ter Gooi Ziekenhuizen, lokatie Blaricum). In augustus 2007 begon als ANIOS urologie in het Maastricht Universitair Medisch Centrum, waar hij vanaf januari 2008 aan zijn promotieonderzoek begon onder begeleiding van Prof. dr. P.E.V. Van Kerrebroeck en Dr. G.A. van Koeveringe. Sinds januari 201 I combineert hij dit onderzoek met klinisch werk als ANIOS longziekten, en later in maart van dat jaar startte hij zijn opleiding tot longarts met een vooropleiding interne geneeskunde tot maart 2013 (Opleider Dr. A. Arntzenius). Vanaf toen tot heden vervolgt hij zijn opleiding tot longarts aan het VUmc onder leiding van Prof. dr. P.E. Postmus en Dr. A. Boonstra. 








